Supporting Information

\title{
Catalytic Dehydrogenative $\beta$-Alkylation of Amino Acid Schiff Bases with Hydrocarbon
}

\author{
Tetsu Ikeda, Haruka Ochiishi, Mana Yoshida, Ryo Yazaki, ${ }^{*}$ and Takashi Ohshima* \\ Graduate School of Pharmaceutical Sciences, Kyushu University, Fukuoka, Japan \\ yazaki@phar.kyushu-u.ac.jp, ohshima@phar.kyushu-u.ac.jp
}

1. General

2. Instrumentation

3. Materials

4. Substrate Syntheses and Characterization

5. General Procedure and Characterization of Products

6. Transformation of the Products

7. References

8. NMR Spectra of Products 


\section{General}

All reactions were carried out using heat gun dried glassware under a positive pressure of dry argon unless otherwise noted. The test tubes were fitted with a 3-way glass stopcock and catalytic reactions were run under argon atmosphere. Air- and moisture-sensitive liquids were transferred via a syringe and a stainlesssteel needle. Reactions were magnetically stirred and monitored by thin layer chromatography using Merck Silica Gel 60 F254 plates. All work-up and purification procedures were carried out with reagentgrade solvents under ambient atmosphere. Flash chromatography was performed using silica gel $60 \mathrm{~N}$ (spherical neutral, particle size $40-50 \mu \mathrm{m}$ ) purchased from Kanto Chemical Co. Ltd.

\section{Instrumentation}

NMR was recorded on $500 \mathrm{MHz}$ Bruker Advanced III. Chemical shifts for proton are reported in parts per million downfield from tetramethylsilane and are referenced to residual protium in the NMR solvent $\left(\mathrm{CDCl}_{3}: \delta 7.26 \mathrm{ppm}\right)$. For ${ }^{13} \mathrm{C} \mathrm{NMR}$, chemical shifts were reported in the scale relative to NMR solvent $\left(\mathrm{CDCl}_{3}: 77.0 \mathrm{ppm}\right)$ as an internal reference. NMR data are reported as follows: chemical shifts, multiplicity ( $\mathrm{s}$ : singlet, d: doublet, dd: doublet of doublets, t: triplet, q: quartet, sep: septet, m: multiplet, br: broad signal), coupling constant $(\mathrm{Hz})$, and integration. Infrared (IR) spectra were recorded on with PerkinElmer Spectrum One FT-IR Spectrometer. High-resolution mass spectroscopy (HRMS) was obtained with Bruker MicrOTOF II. Optical rotation was measured with JASCO DIP-370 polarimeter.

\section{Materials}

Cumene was distilled after dried with $\mathrm{CaH}_{2}$ and stored in schlenk flask. Deoxidized toluene was purchased from Wako Pure Chemical Industries (Wako) and used as received. o-Dichlorobenzene was dried over MS4A. Copper(II) acetate was purchased from Wako Pure Chemical Industries (Wako) and stored in an inert atmosphere glove box. DTBP was purchased from $\mathrm{TCl}$ and used as received. All other commercially available reagents were used as received. 


\section{Substrate Syntheses and Characterization}

\section{General Procedure A (1a-1d) ${ }^{1}$}

A flame-dried $25 \mathrm{~mL}$ round-bottom flask equipped with a magnetic stirring bar was filled with argon, and charged with the corresponding amino acid methyl ester hydrochloride (1.0 equiv), benzophenone imine (1.0 equiv) and $\mathrm{CH}_{2} \mathrm{Cl}_{2}(1.0 \mathrm{M})$. The mixture was stirred overnight at ambient temperature. After the reaction mixture was filtered, the solution was poured into sat. $\mathrm{NaHCO}_{3}$ aq and extracted with $\mathrm{CH}_{2} \mathrm{Cl}_{2}$. The combined organic layers were washed with brine, dried over sodium sulfate, and filtrated then purified by flash column chromatography.<smiles>COC(=O)C(C)N=C(c1ccccc1)c1ccccc1</smiles>

Methyl 2-((diphenylmethylene)amino)propanoate (1a); CAS Registry Number 195600-97-6

Prepared according to General Method $\mathrm{A}$ and purified by flash column chromatography $\left(\mathrm{Hexane} / \mathrm{Et}_{2} \mathrm{O}=\right.$ $5 / 1\left(1 \% \mathrm{Et}_{3} \mathrm{~N}\right), 2.32 \mathrm{~g}, 87 \%$ yield, white solid); ${ }^{1} \mathrm{H} \mathrm{NMR}\left(500 \mathrm{MHz}, \mathrm{CDCl}_{3}, 27^{\circ} \mathrm{C}\right) \delta 7.64(\mathrm{~d}, J=7.0 \mathrm{~Hz}, 2 \mathrm{H}$, $\operatorname{ArH}), 7.48-7.44(\mathrm{~m}, 3 \mathrm{H}, \operatorname{ArH}), 7.37(\mathrm{t}, J=7.5 \mathrm{~Hz}, 1 \mathrm{H}, \operatorname{ArH}), 7.33(\mathrm{t}, J=8.5 \mathrm{~Hz}, 2 \mathrm{H}, \operatorname{ArH}), 6.19(\mathrm{~d}, J=8.0 \mathrm{~Hz}$, $2 \mathrm{H}, \operatorname{ArH}), 4.18(\mathrm{q}, J=7.0 \mathrm{~Hz}, 1 \mathrm{H}, \mathrm{CH}), 3.72\left(\mathrm{~s}, 3 \mathrm{H}, \mathrm{OCH}_{3}\right), 1.42\left(\mathrm{~d}, J=6.5 \mathrm{~Hz}, 3 \mathrm{H}, \mathrm{CH}_{3}\right) ;{ }^{13} \mathrm{C} \mathrm{NMR}(125 \mathrm{MHz}$, $\left.\mathrm{CDCl}_{3}, 27^{\circ} \mathrm{C}\right) \delta 173.5,169.7,139.5,136.2,130.3,128.8,128.7,128.6,128.1,127.7,60.6,52.1,19.2$.<smiles>CCC(N=C(c1ccccc1)c1ccccc1)C(=O)OC</smiles>

Methyl 2-((diphenylmethylene)amino)butanoate (1b); CAS Registry Number 553645-47-9

Prepared according to General Method $\mathrm{A}$ and purified by flash column chromatography (Hexane/Et ${ }_{2} \mathrm{O}=$ 5/1 (1\% Et $3 \mathrm{~N}), 0.78 \mathrm{~g}, 56 \%$ yield, colorless oil); ${ }^{1} \mathrm{H}$ NMR $\left(500 \mathrm{MHz}, \mathrm{CDCl}_{3}, 2{ }^{\circ} \mathrm{C}\right) \delta 7.65(\mathrm{~d}, J=7.0 \mathrm{~Hz}, 2 \mathrm{H}$, $\operatorname{ArH})$, 7.47-7.45 (m, 3H, ArH), 7.44-7.40 (m, 1H, ArH), $7.33(\mathrm{t}, J=7.0 \mathrm{~Hz}, 2 \mathrm{H}, \operatorname{ArH})$, 7.18-7.16 (m, 2H, $\operatorname{ArH})$, $4.01(\mathrm{dd}, J=8.0,5.0 \mathrm{~Hz}, 1 \mathrm{H}, \mathrm{CH}), 3.72\left(\mathrm{~s}, 3 \mathrm{H}, \mathrm{OCH}_{3}\right), 2.00-1.85\left(\mathrm{~m}, 2 \mathrm{H}, \mathrm{CH}_{2}\right), 0.85\left(\mathrm{t}, J=7.5 \mathrm{~Hz}, 3 \mathrm{H}, \mathrm{CH}_{3}\right) ;{ }^{13} \mathrm{C}$ $\operatorname{NMR}\left(125 \mathrm{MHz} \mathrm{CDCl}_{3}, 27^{\circ} \mathrm{C}\right) \delta 172.9,170.4,139.6,136.5,130.3,128.8,128.6,128.5,128.1,127.9,66.8$, 52.0, 27.0, 10.5. 
<smiles>COC(=O)C(Cc1ccccc1)N=C(c1ccccc1)c1ccccc1</smiles>

Methyl 2-((diphenylmethylene)amino)-3-phenylpropanoate (1c); CAS Registry Number 120238-42-8

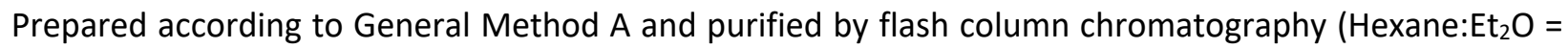
5:1 (1\% Et $3 \mathrm{~N}), 1.44 \mathrm{~g}, 84 \%$ yield, white solid); ${ }^{1} \mathrm{H} \mathrm{NMR}\left(500 \mathrm{MHz}, \mathrm{CDCl}_{3}, 27{ }^{\circ} \mathrm{C}\right) \delta 7.58(\mathrm{~d}, J=7.0 \mathrm{~Hz}, 2 \mathrm{H}$, $\operatorname{ArH})$, 7.39-7.27 (m, 6H, ArH), 7.20-7.14 (m, 3H, ArH), 7.02 (dd, J = 7.5, 2.0 Hz, 2H, ArH), 7.03-7.01 (m, 2H, $\operatorname{ArH}), 4.26(\mathrm{dd}, J=9.5,4.5 \mathrm{~Hz}, 1 \mathrm{H}, \mathrm{CH}), 3.73\left(\mathrm{~s}, 3 \mathrm{H}, \mathrm{OCH}_{3}\right), 3.27$ (dd, $J=13.5,4.0 \mathrm{~Hz}, 1 \mathrm{H}, \mathrm{ArCH}_{2}$ ), 3.17 (dd, $J$ = 13.0, $\left.9.5 \mathrm{~Hz}, 1 \mathrm{H}, \mathrm{ArCH}_{2}\right) ;{ }^{13} \mathrm{C} \mathrm{NMR}\left(125 \mathrm{MHz}, \mathrm{CDCl}_{3}, 27^{\circ} \mathrm{C}\right) \delta 172.3,170.9,139.4,137.9,136.1,130.3$, $129.9,128.8,128.3,128.2,128.2,128.0,127.6,126.3,67.3,52.2,39.8$.

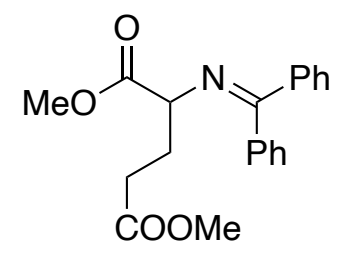

Dimethyl 2-((diphenylmethylene)amino)pentanedioate (1d) ; CAS Registry Number 221035-75-2

Prepared according to General Method $A$ and purified by flash column chromatography $\left(\mathrm{Hexane}_{\mathrm{Et}} \mathrm{O}=\right.$ 5/1 (1\% Et $3 \mathrm{~N}), 1.51 \mathrm{~g}, 89 \%$ yield, colorless oil); ${ }^{1} \mathrm{H} \mathrm{NMR}\left(500 \mathrm{MHz}, \mathrm{CDCl}_{3}, 2{ }^{\circ} \mathrm{C}\right) \delta 7.63(\mathrm{~d}, J=7.5 \mathrm{~Hz}, 2 \mathrm{H}$, $\operatorname{ArH})$, 7.46-7.43 (m, 3H, ArH), $7.40(\mathrm{t}, J=7.5 \mathrm{~Hz}, 1 \mathrm{H}, \operatorname{ArH}), 7.33(\mathrm{t}, J=7.5 \mathrm{~Hz}, 2 \mathrm{H}, \operatorname{ArH}), 7.19-7.17(\mathrm{~m}, 2 \mathrm{H}$, $\operatorname{ArH}), 4.13(\mathrm{t}, J=7.0 \mathrm{~Hz}, 1 \mathrm{H}, \mathrm{CH}), 3.71\left(\mathrm{~s}, 3 \mathrm{H}, \mathrm{OCH}_{3}\right), 3.58\left(\mathrm{~s}, 3 \mathrm{H}, \mathrm{OCH}_{3}\right), 2.36\left(\mathrm{t}, J=7.5 \mathrm{~Hz}, 2 \mathrm{H}, \mathrm{CH}_{2} \mathrm{COOCH}_{3}\right)$, $2.24\left(\mathrm{q}, J=7.5 \mathrm{~Hz}, 2 \mathrm{H}, \mathrm{CH}_{2}\right) ;{ }^{13} \mathrm{C}$ NMR $\left(125 \mathrm{MHz}, \mathrm{CDCl}_{3}, 27^{\circ} \mathrm{C}\right) \delta 173.4,172.2,171.2,139.3,136.1,130.5$, 128.9, 128.7, 128.6, 128.1, 127.8, 64.1, 52.2, 51.5, 30.4, 28.6. 


\section{General Procedure and Characterization of Products}

\section{5-2. $\beta$-Alkylation}

\section{General Procedure B}

In an inert atmosphere glove box, a $4 \mathrm{ml}$ light resident vial was charged with copper(II) acetate (0.020 $\mathrm{mmol}, 10 \mathrm{~mol} \%)$ and removed from the glovebox. To the vial was added $\alpha$-amino acid Schiff base 1 (0.20 mmol, 1.0 equiv) and coupling partner 2 ( $7.2 \mathrm{mmol}, 36$ equiv) followed by DTBP ( $0.66 \mathrm{mmol}, 3.3$ equiv) under argon atmosphere. The resultant reaction mixture was stirred at $100{ }^{\circ} \mathrm{C}$ using heating block for 24 hours. After cooling down to room temperature, copper salt was removed by silica gel short pad column chromatography and washed with excess amount of ethyl acetate. After evaporation of the organic solvent under reduced pressure, the resultant mixture was purified by silica gel flash chromatography.

\section{General Procedure C}

In an inert atmosphere glove box, a $4 \mathrm{ml}$ light resident vial was charged with copper(II) acetate (0.030 $\mathrm{mmol}, 15 \mathrm{~mol} \%)$ and removed from the glovebox. To the vial was added $\alpha$-amino acid Schiff base 1 (0.20 mmol, 1.0 equiv) and coupling partner $2(0.30 \mathrm{ml})$ followed by DTBP ( $0.60 \mathrm{mmol}, 3.0$ equiv) under argon atmosphere. The resultant reaction mixture was stirred at $100{ }^{\circ} \mathrm{C}$ using heating block for 24 hours. After cooling down to room temperature, copper salt was removed by silica gel short pad column chromatography and washed with excess amount of ethyl acetate. After evaporation of the organic solvent under reduced pressure, the resultant mixture was purified by silica gel flash chromatography.

\section{General Procedure D}

In an inert atmosphere glove box, a $4 \mathrm{ml}$ light resident vial was charged with copper(II) acetate (0.020 $\mathrm{mmol}, 10 \mathrm{~mol} \%)$ and removed from the glovebox. To the vial was added alanine Schiff base $(0.20 \mathrm{mmol}$, 1.0 equiv), coupling partner $2(2.0 \mathrm{mmol}, 10$ equiv), and $o$-dichlorobenzene $(1.0 \mathrm{ml}, 0.20 \mathrm{M})$ followed by DTBP $\left(0.66 \mathrm{mmol}, 3.3\right.$ equiv) under argon atmosphere. The resultant reaction mixture was stirred at $100^{\circ} \mathrm{C}$ using heating block for 24 hours. After cooling down to room temperature, copper salt was removed by silica gel short pad column chromatography and washed with excess amount of ethyl acetate. After evaporation of the organic solvent under reduced pressure, the resultant mixture was purified by silica gel flash chromatography. 
<smiles>COC(=O)/C(=C/C(C)(C)c1ccccc1)Nc1ccccc1</smiles>

Methyl (Z)-2-((diphenylmethylene)amino)-4-methyl-4-phenylpent-2-enoate (3aa): (Yellow oil, $n$ Hexane/Diethyl ether = 20/1 to 4/1, General Procedure B (1.1 mmol scale): 76\% yield, $322.0 \mathrm{mg}$ ); IR (neat) 1716, 1444, 1240, $1085 \mathrm{~cm}^{-1} ;{ }^{1} \mathrm{H}$ NMR $\left(500 \mathrm{MHz}, \mathrm{CDCl}_{3}, 27^{\circ} \mathrm{C}\right) \delta 7.55(\mathrm{~d}, J=7.0 \mathrm{~Hz}, 2 \mathrm{H}, \operatorname{ArH}), 7.43(\mathrm{t}, J=$ $7.25 \mathrm{~Hz}, 1 \mathrm{H}, \mathrm{ArH}), 7.35-7.27(\mathrm{~m}, 6 \mathrm{H}, \mathrm{ArH}), 7.21(\mathrm{t}, J=7.7 \mathrm{~Hz}, 2 \mathrm{H}, \mathrm{ArH}), 7.13$ (triplet of triplets, $J=7.25,1.5$, $1.25 \mathrm{~Hz}, 1 \mathrm{H}, \mathrm{ArH}), 6.88(\mathrm{~d}, J=7.0 \mathrm{~Hz}, 2 \mathrm{H}, \mathrm{ArH}), 6.33(\mathrm{~s}, 1 \mathrm{H}, \mathrm{CH}), 3.42\left(\mathrm{~s}, 3 \mathrm{H}, \mathrm{OCH}_{3}\right), 1.57\left(\mathrm{~s}, 6 \mathrm{H}, \mathrm{CH}_{3}\right) ;{ }^{13} \mathrm{C}$ $\operatorname{NMR}\left(125 \mathrm{MHz}, \mathrm{CDCl}_{3}, 27^{\circ} \mathrm{C}\right) \delta 168.6,165.4,148.6,138.8,137.5,136.8,135.1,130.7,129.4,128.9,128.2$, 128.0, 128.0, 127.8, 126.3, 125.7, 51.7, 39.9, 28.9; HRMS (ESI) m/z: $[\mathrm{M}+\mathrm{H}]^{+}$calcd. for $\mathrm{C}_{26} \mathrm{H}_{26} \mathrm{NO}_{2}{ }^{+} 384.1958$ found 384.1959 .

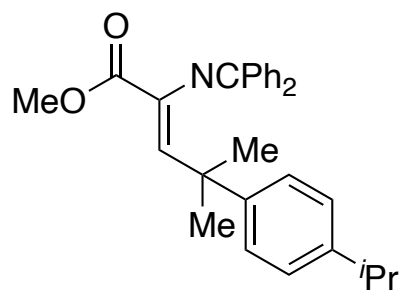

Methyl (Z)-2-((diphenylmethylene)amino)-4-(4-isopropylphenyl)-4-methylpent-2-enoate (3ab): (Pale yellow oil, $n$-Hexane/Dichloromethane/Diethyl ether = 73/20/2, General Procedure C: 61\% yield, $52.5 \mathrm{mg}$ ); IR (neat) 2958, 1716, 1622, 1240, 1080, $1001 \mathrm{~cm}^{-1} ;{ }^{1} \mathrm{H} \mathrm{NMR}\left(500 \mathrm{MHz}, \mathrm{CDCl}_{3}, 27^{\circ} \mathrm{C}\right) \delta 7.54$ (d, J = $7.5 \mathrm{~Hz}$, $2 \mathrm{H}, \operatorname{ArH}), 7.43(\mathrm{t}, J=7.25 \mathrm{~Hz}, 1 \mathrm{H}, \operatorname{ArH}), 7.35-7.30(\mathrm{~m}, 3 \mathrm{H}, \mathrm{ArH}), 7.27-7.22(\mathrm{~m}, 4 \mathrm{H}, \operatorname{ArH}), 7.06(\mathrm{~d}, J=8.0 \mathrm{~Hz}$, $2 \mathrm{H}, \operatorname{ArH}), 6.84(\mathrm{~d}, J=7.5 \mathrm{~Hz}, 2 \mathrm{H}, \mathrm{ArH}), 6.32(\mathrm{~s}, 1 \mathrm{H}, \mathrm{CH}), 3.42\left(\mathrm{~s}, 3 \mathrm{H}, \mathrm{OCH}_{3}\right), 2.83(\mathrm{sep}, J=6.83 \mathrm{~Hz}, 1 \mathrm{H}, \mathrm{CH})$, $1.56\left(\mathrm{~s}, 6 \mathrm{H}, \mathrm{CH}_{3}\right), 1.19\left(\mathrm{~d}, J=6.5 \mathrm{~Hz}, 6 \mathrm{H}, \mathrm{CH}_{3}\right) ;{ }^{13} \mathrm{CNMR}\left(125 \mathrm{MHz}, \mathrm{CDCl}_{3}, 27^{\circ} \mathrm{C}\right) \delta 168.5,165.4,146.2,145.8$, $138.8,137.4,136.8,135.4,130.6,129.3,128.8,128.2$, 127.9, 127.7, 126.2, 126.0, 51.6, 39.5, 33.6, 28.9, 24.0; HRMS (ESI) m/z: [M + H] $]^{+}$calcd. for $\mathrm{C}_{29} \mathrm{H}_{32} \mathrm{NO}_{2}{ }^{+} 426.2428$ found 426.2429. 


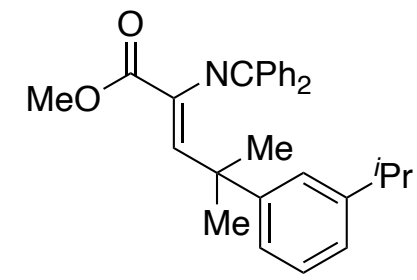

Methyl (Z)-2-((diphenylmethylene)amino)-4-(3-isopropylphenyl)-4-methylpent-2-enoate (3ac): (Pale yellow oil, $n$-Hexane/Dichloromethane/Diethyl ether = 30/15/1, General Procedure C: 52\% yield, $43.9 \mathrm{mg}$ ); IR (neat) 2960, 1718, 1624, 1444, 1236, $1068 \mathrm{~cm}^{-1} ;{ }^{1} \mathrm{H}$ NMR $\left(500 \mathrm{MHz}, \mathrm{CDCl}_{3}, 27^{\circ} \mathrm{C}\right) \delta 7.58(\mathrm{~d}, J=7.5 \mathrm{~Hz}$, $2 \mathrm{H}, \operatorname{ArH}), 7.42(\mathrm{t}, J=7.0 \mathrm{~Hz}, 1 \mathrm{H}, \operatorname{ArH}), 7.35-7.23(\mathrm{~m}, 5 \mathrm{H}, \mathrm{ArH}), 7.17-7.13(\mathrm{~m}, 3 \mathrm{H}, \operatorname{ArH}), 7.01-6.99(\mathrm{~m}, 1 \mathrm{H}$, $\left.\operatorname{ArH}), 6.82(\mathrm{~d}, J=7.5 \mathrm{~Hz}, 2 \mathrm{H}, \operatorname{ArH}), 6.34(\mathrm{~s}, 1 \mathrm{H}, \mathrm{CH}), 3.40(\mathrm{~s}, 3 \mathrm{H}, \mathrm{OCH})_{3}\right), 2.76(\mathrm{sep}, J=7.0 \mathrm{~Hz}, 1 \mathrm{H}, \mathrm{CH}), 1.60$ (s, 6H, $\left.\mathrm{CH}_{3}\right), 1.13$ (d, J = 7.0 Hz, 6H, $\left.\mathrm{CH}_{3}\right) ;{ }^{13} \mathrm{C}$ NMR $\left(125 \mathrm{MHz}, \mathrm{CDCl}_{3}, 2{ }^{\circ} \mathrm{C}\right) \delta 168.5,165.3,148.5,148.4$, $138.8,137.5,136.8,135.6,130.6,129.4,128.9,128.2$, 128.0, 127.7, 124.3, 124.2, 123.5, 51.6, 39.8, 34.2, 28.8, 24.0; HRMS (ESI) m/z: [M + H] calcd. for $\mathrm{C}_{29} \mathrm{H}_{32} \mathrm{NO}_{2}{ }^{+} 426.2428$ found 426.2430 .

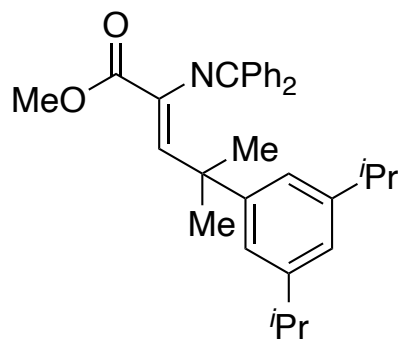

Methyl (Z)-4-(3,5-diisopropylphenyl)-2-((diphenylmethylene)amino)-4-methylpent-2-enoate (3ad):

(Pale yellow oil, $n$-Hexane/Ethyl acetate $=20 / 1$, General Procedure C: 48\% yield, $45.0 \mathrm{mg}$ ); IR (neat) 2956, 1718, 1595, $1236 \mathrm{~cm}^{-1} ;{ }^{1} \mathrm{H}$ NMR $\left(500 \mathrm{MHz}, \mathrm{CDCl}_{3}, 27^{\circ} \mathrm{C}\right) \delta 7.61(\mathrm{~d}, J=7.0 \mathrm{~Hz}, 2 \mathrm{H}, \mathrm{ArH}), 7.42(\mathrm{t}, J=7.25 \mathrm{~Hz}$, $1 \mathrm{H}, \operatorname{ArH}), 7.35-7.28(\mathrm{~m}, 3 \mathrm{H}, \mathrm{ArH}), 7.23(\mathrm{t}, J=7.25 \mathrm{~Hz}, 2 \mathrm{H}, \operatorname{ArH}), 7.01(\mathrm{~d}, J=1,55 \mathrm{~Hz}, 2 \mathrm{H}, \operatorname{ArH}), 6.86(\mathrm{~s}, 1 \mathrm{H}$, $\operatorname{ArH}), 6.78(\mathrm{~d}, J=7.5 \mathrm{~Hz}, 2 \mathrm{H}, \mathrm{ArH}), 6.34(\mathrm{~s}, 1 \mathrm{H}, \mathrm{CH}), 3.38\left(\mathrm{~s}, 3 \mathrm{H}, \mathrm{OCH}_{3}\right), 2.74(\mathrm{sep}, J=6.92 \mathrm{~Hz}, 1 \mathrm{H}, \mathrm{CH}), 1.62$ $\left(\mathrm{s}, 6 \mathrm{H}, \mathrm{CH}_{3}\right), 1.13\left(\mathrm{~d}, J=7.0 \mathrm{~Hz}, 12 \mathrm{H}, \mathrm{CH}_{3}\right) ;{ }^{13} \mathrm{C} \mathrm{NMR}\left(125 \mathrm{MHz}, \mathrm{CDCl}_{3}, 27^{\circ} \mathrm{C}\right) \delta 168.3,165.3,148.4,148.2$, 138.7, 137.4, 136.8, 136.1, 130.6, 129.4, 128.8, 128.2, 128.0, 127.7, 122.2, 121.6, 51.5, 39.7, 34.3, 28.7, 24.1; HRMS (ESI) m/z: [M+ H] $]^{+}$calcd. for $\mathrm{C}_{32} \mathrm{H}_{38} \mathrm{NO}_{2}{ }^{+} 468.2897$ found 468.2897. 


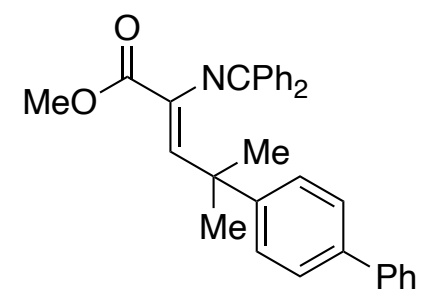

Methyl (Z)-4-([1,1'-biphenyl]-4-yl)-2-((diphenylmethylene)amino)-4-methylpent-2-enoate (3ae): (Pale yellow solid, $n$-Hexane/Dichloromethane/Diethyl ether $=30 / 10 / 1$, General Procedure C: 47\% yield, 43.4 $\mathrm{mg}$ ); IR (neat) 1716, 1597, 1485, $1236 \mathrm{~cm}^{-1} ;{ }^{1} \mathrm{H}$ NMR (500 MHz, CDCl, $\left.27^{\circ} \mathrm{C}\right) \delta ; 7.52$ (t, J=7.25 Hz, 4H, $\operatorname{ArH})$, 7.45-7.37 (m, 9H, ArH), 7.33-7.27 (m, 7H, ArH), $6.93(\mathrm{~d}, J=7.0 \mathrm{~Hz}, 2 \mathrm{H}, \mathrm{ArH}), 6.35(\mathrm{~s}, 1 \mathrm{H}, \mathrm{CH}), 3.45(\mathrm{~s}$, $\left.3 \mathrm{H}, \mathrm{OCH}_{3}\right), 1.60\left(\mathrm{~s}, 6 \mathrm{H}, \mathrm{CH}_{3}\right) ;{ }^{13} \mathrm{C} \mathrm{NMR}\left(125 \mathrm{MHz}, \mathrm{CDCl}_{3}, 27^{\circ} \mathrm{C}\right) \delta 168.5,165.4,147.7,141.1,138.8,138.6$, 137.6, 136.8, 134.9, 130.7, 129.4, 129.0, 128.7, 128.2, 128.0, 127.8, 127.0, 127.0, 126.8, 51.7, 39.7, 29.1; HRMS (ESI) m/z: [M + H] calcd. for $\mathrm{C}_{32} \mathrm{H}_{30} \mathrm{NO}_{2}{ }^{+} 460.2271$ found 460.2271 .

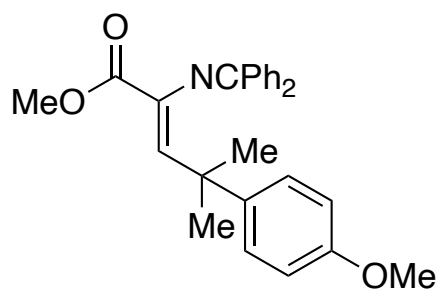

Methyl (Z)-2-((diphenylmethylene)amino)-4-(4-methoxyphenyl)-4-methylpent-2-enoate (3af): (Pale yellow oil, $n$-Hexane/Dichloromethane/Diethyl ether = 20/30/1, General Procedure B: 26\% yield, 21.9 $\mathrm{mg}) ; \mathrm{IR}$ (neat) 1716, 1608, 1508, 1244, 1180, $1031 \mathrm{~cm}^{-1} ;{ }^{1} \mathrm{H} \mathrm{NMR}\left(500 \mathrm{MHz}, \mathrm{CDCl}_{3}, 27^{\circ} \mathrm{C}\right) \delta 7.56$ (d, $J=7.5$ $\mathrm{Hz}, 2 \mathrm{H}, \mathrm{ArH}), 7.43(\mathrm{t}, J=7.25 \mathrm{~Hz}, 1 \mathrm{H}, \operatorname{ArH}), 7.35-7.27(\mathrm{~m}, 5 \mathrm{H}, \mathrm{ArH}), 7.22(\mathrm{dt}, J=9.0,2.75 \mathrm{~Hz}, 2 \mathrm{H}, \operatorname{ArH}), 6.92$ $(\mathrm{d}, J=7.5 \mathrm{~Hz}, 2 \mathrm{H}, \mathrm{ArH}), 6.75(\mathrm{dt}, J=9.0,2.5 \mathrm{~Hz}, 2 \mathrm{H}, \mathrm{ArH}), 6.29(\mathrm{~s}, 1 \mathrm{H}, \mathrm{CH}), 3.74\left(\mathrm{~s}, 3 \mathrm{H}, \mathrm{OCH}_{3}\right), 3.43(\mathrm{~s}, 3 \mathrm{H}$, $\left.\mathrm{OCH}_{3}\right), 1.54\left(\mathrm{~s}, 6 \mathrm{H}, \mathrm{CH}_{3}\right) ;{ }^{13} \mathrm{C} \mathrm{NMR}\left(125 \mathrm{MHz}, \mathrm{CDCl}_{3}, 27^{\circ} \mathrm{C}\right) \delta 168.5,165.4,157.6,140.8,138.8,137.3,136.8$, $135.2,130.6,129.4,128.9,128.2,127.9,127.8,127.3,113.4,55.2,51.6,39.3,29.1 ;$ HRMS (ESI) m/z: [M + $\mathrm{H}]^{+}$calcd. for $\mathrm{C}_{27} \mathrm{H}_{28} \mathrm{NO}_{3}{ }^{+} 414.2064$ found 414.2064. 
<smiles>CCN(C)/C(=C\C(C)(C)c1ccc(Br)cc1)C(=O)OC</smiles>

Methyl (Z)-4-(4-bromophenyl)-2-((diphenylmethylene)amino)-4-methylpent-2-enoate (3ag): (Pale yellow oil, $n$-Hexane/Dichloromethane/Diethyl ether = 30/10/1, General Procedure C: 72\% yield, $67.5 \mathrm{mg}$ ); IR (neat) $1716,1624,1232,1006 \mathrm{~cm}^{-1} ;{ }^{1} \mathrm{H}$ NMR $\left(500 \mathrm{MHz}, \mathrm{CDCl}_{3}, 27^{\circ} \mathrm{C}\right) \delta$ 7.47-7.42 (m, 3H, ArH), 7.35-7.29 $(\mathrm{m}, 7 \mathrm{H}, \operatorname{ArH}), 7.16(\mathrm{dt}, J=2.5,8.5 \mathrm{~Hz}, 2 \mathrm{H}, \operatorname{ArH}), 6.95(\mathrm{~d}, J=7.0 \mathrm{~Hz}, 2 \mathrm{H}, \operatorname{ArH}), 6.27(\mathrm{~s}, 1 \mathrm{H}, \mathrm{CH}), 3.43(\mathrm{~s}, 3 \mathrm{H}$, $\left.\mathrm{OCH}_{3}\right), 1.51\left(\mathrm{~s}, 6 \mathrm{H}, \mathrm{CH}_{3}\right) ;{ }^{13} \mathrm{C} \mathrm{NMR}\left(125 \mathrm{MHz}, \mathrm{CDCl}_{3}, 27^{\circ} \mathrm{C}\right) \delta 168.4,165.3,147.6,138.6,137.8,136.7,134.3$, 131.0, 130.7, 129.4, 129.1, 128.2, 128.0, 127.9, 119.5, 51.7, 39.6, 29.1; HRMS (ESI) m/z: [M + H] calcd. for $\mathrm{C}_{26} \mathrm{H}_{25} \mathrm{BrNO}_{2}{ }^{+} 462.1063$ found 462.1060 .<smiles>COC(=O)/C(=C/C(C)(C)c1ccc(Cl)cc1)N(C)c1ccccc1</smiles>

Methyl (Z)-4-(4-acetylphenyl)-2-((diphenylmethylene)amino)-4-methylpent-2-enoate (3ah): (Pale yellow oil, $n$-Hexane/Dichloromethane/Diethyl ether $=66 / 9 / 21$, General Procedure C: ${ }^{1} \mathrm{H}-\mathrm{NMR}$ yield (2methoxynaphthalene as an internal standard): 50\%, Isolated yield: $24 \%$ yield, $20.9 \mathrm{mg}$ ); IR (neat) 1716, $1681,1602,1267,1236 \mathrm{~cm}^{-1} ;{ }^{1} \mathrm{H}$ NMR $\left(500 \mathrm{MHz}, \mathrm{CDCl}_{3}, 27^{\circ} \mathrm{C}\right) \delta 7.79(\mathrm{~d}, J=8.5 \mathrm{~Hz}, 2 \mathrm{H}, \mathrm{ArH}), 7.43-7.39(\mathrm{~m}$, 5H, ArH), 7.35-7.28 (m, 5H, ArH), $6.94(\mathrm{~d}, J=7.0 \mathrm{~Hz}, 2 \mathrm{H}, \mathrm{ArH}), 6.32(\mathrm{~s}, 1 \mathrm{H}, \mathrm{CH}), 3.41\left(\mathrm{~s}, 3 \mathrm{H}, \mathrm{OCH}_{3}\right), 2.52(\mathrm{~s}$, $\left.3 \mathrm{H}, \mathrm{CH}_{3}\right), 1.57\left(\mathrm{~s}, 6 \mathrm{H}, \mathrm{CH}_{3}\right) ;{ }^{13} \mathrm{C}$ NMR $\left(125 \mathrm{MHz}, \mathrm{CDCl}_{3}, 27^{\circ} \mathrm{C}\right) \delta 197.8,168.3,165.2,154.3,138.5,138.0$, 136.6, 134.7, 134.4, 130.8, 129.4, 129.1, 128.2, 128.2, 127.9, 126.5, 51.7, 40.2, 29.0, 26.5; HRMS (ESI) m/z: $[\mathrm{M}+\mathrm{H}]^{+}$calcd. for $\mathrm{C}_{28} \mathrm{H}_{28} \mathrm{NO}_{3}{ }^{+} 426.2064$ found 426.2065. 


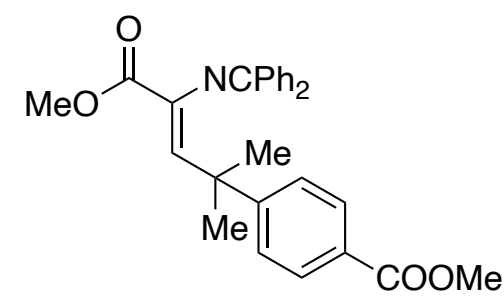

Methyl (Z)-4-(4-((diphenylmethylene)amino)-5-methoxy-2-methyl-5-oxopent-3-en-2-yl)benzoate (3ai):

(Pale yellow oil, $n$-Hexane/Ethyl acetate $=8 / 1$, General Procedure C: 41\% yield, $36.4 \mathrm{mg}$ ); IR (neat) 1716, 1606, 1433, 1276, 1238, $1112 \mathrm{~cm}^{-1} ;{ }^{1} \mathrm{H}$ NMR $\left(500 \mathrm{MHz}, \mathrm{CDCl}_{3}, 27^{\circ} \mathrm{C}\right) \delta 7.87(\mathrm{dt}, J=8.5,2.0 \mathrm{~Hz}, 2 \mathrm{H}, \operatorname{ArH})$, $7.65(\mathrm{~d}, J=8.0 \mathrm{~Hz}, 2 \mathrm{H}, \mathrm{ArH}), 7.42(\mathrm{t}, J=7.5 \mathrm{~Hz}, 1 \mathrm{H}, \operatorname{ArH}), 7.34-7.27(\mathrm{~m}, 5 \mathrm{H}, \mathrm{ArH}), 7.37(\mathrm{dt}, J=8.5,2.0 \mathrm{~Hz}$, $2 \mathrm{H}, \operatorname{ArH}), 6.92(\mathrm{~d}, J=7.0 \mathrm{~Hz}, 2 \mathrm{H}, \mathrm{ArH}), 6.32(\mathrm{~s}, 1 \mathrm{H}, \mathrm{CH}), 3.89\left(\mathrm{~s}, 3 \mathrm{H}, \mathrm{OCH}_{3}\right), 3.41\left(\mathrm{~s}, 3 \mathrm{H}, \mathrm{OCH}_{3}\right), 1.57(\mathrm{~s}, 6 \mathrm{H}$, $\left.\mathrm{CH}_{3}\right) ;{ }^{13} \mathrm{C}$ NMR $\left(125 \mathrm{MHz}, \mathrm{CDCl}_{3}, 2{ }^{\circ} \mathrm{C}\right) \delta 168.5,167.1,165.2,154.0,138.5,138.0,136.7,134.4,130.8$, 129.4, 129.4, 129.1, 128.2, 127.9, 127.9, 127.5, 126.3, 51.9, 51.7, 40.1, 29.0; HRMS (ESI) m/z: [M + H] calcd. for $\mathrm{C}_{28} \mathrm{H}_{28} \mathrm{NO}_{4}{ }^{+} 442.2013$ found 442.2014 .<smiles>COC(=O)/C(=C/C(C)(C)c1ccc2ccccc2c1)N(C)c1ccccc1</smiles>

Methyl (Z)-2-((diphenylmethylene)amino)-4-methyl-4-(naphthalen-2-yl)pent-2-enoate (3aj): (Pale yellow oil, $n$-Hexane/Dichloromethane/Diethyl ether $=40 / 10 / 1$, General Procedure C: $45 \%$ yield, $38.8 \mathrm{mg}$ ); IR (neat) 1716, 1597, $1242 \mathrm{~cm}^{-1} ;{ }^{1} \mathrm{H}$ NMR $\left(500 \mathrm{MHz}, \mathrm{CDCl}_{3}, 27^{\circ} \mathrm{C}\right) \delta 7.77-7.73(\mathrm{~m}, 2 \mathrm{H}, \mathrm{ArH}), 7.65(\mathrm{~d}, \mathrm{~J}=1.0$ $\mathrm{Hz}, 1 \mathrm{H}, \mathrm{ArH}), 7.63-7.61(\mathrm{~m}, 1 \mathrm{H}, \mathrm{ArH}), 7.51(\mathrm{dd}, J=8.5,2.0 \mathrm{~Hz}, 1 \mathrm{H}, \operatorname{ArH}), 7.41-7.34(\mathrm{~m}, 5 \mathrm{H}, \operatorname{ArH}), 7.29(\mathrm{t}, J=$ $7.5 \mathrm{~Hz}, 1 \mathrm{H}, \mathrm{ArH}), 7.23-7.16(\mathrm{~m}, 4 \mathrm{H}, \mathrm{ArH}), 6.88(\mathrm{~d}, J=7.5 \mathrm{~Hz}, 2 \mathrm{H}, \mathrm{ArH}), 6.40(\mathrm{~s}, 1 \mathrm{H}, \mathrm{CH}), 3.43(\mathrm{~s}, 3 \mathrm{H}, \mathrm{OCH})$, $1.65\left(\mathrm{~s}, 6 \mathrm{H}, \mathrm{CH}_{3}\right) ;{ }^{13} \mathrm{C} \mathrm{NMR}\left(125 \mathrm{MHz}, \mathrm{CDCl}_{3}, 27^{\circ} \mathrm{C}\right) \delta 168.4,165.4,145.9,138.6,137.8,136.7,134.8,133.3$, 131.9, 130.6, 129.3, 128.9, 128.1, 127.9, 127.8, 127.7, 127.6, 127.3, 125.8, 125.7, 125.2, 123.9, 51.7, 40.0, 29.0; HRMS (ESI) m/z: [M+ H] $]^{+}$calcd. for $\mathrm{C}_{30} \mathrm{H}_{28} \mathrm{NO}_{2}{ }^{+} 434.2115$ found 434.2112. 


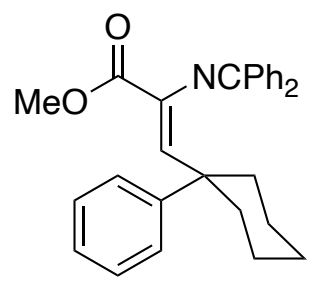

Methyl (Z)-2-((diphenylmethylene)amino)-3-(1-phenylcyclohexyl)acrylate (3ak): (Pale yellow oil, $n$ Hexane/Dichloromethane/Diethyl ether = 40/10/1, General Procedure C: 11\% yield, $9.4 \mathrm{mg}$ ); IR (neat) 2942, 1716, 1444, 1238, $1193 \mathrm{~cm}^{-1} ;{ }^{1} \mathrm{H}$ NMR $\left(500 \mathrm{MHz}, \mathrm{CDCl}_{3}, 27^{\circ} \mathrm{C}\right) \delta 7.56$ (d, J = $\left.7.5 \mathrm{~Hz}, 2 \mathrm{H}, \operatorname{ArH}\right), 7.44$ $(\mathrm{t}, J=7.25 \mathrm{~Hz}, 1 \mathrm{H}, \operatorname{ArH}), 7.35(\mathrm{t}, J=7.75 \mathrm{~Hz}, 2 \mathrm{H}, \operatorname{ArH}), 7.29$ (d, J=7.5 Hz, 2H, ArH), $7.23(\mathrm{t}, J=7.25 \mathrm{~Hz}, 1 \mathrm{H}$, $\operatorname{ArH}), 7.18(\mathrm{t}, J=7.5 \mathrm{~Hz}, 2 \mathrm{H}, \operatorname{ArH}), 7.15-7.09(\mathrm{~m}, 3 \mathrm{H}, \operatorname{ArH}), 6.50(\mathrm{~d}, J=7.5 \mathrm{~Hz}, 2 \mathrm{H}, \operatorname{ArH}), 6.34(\mathrm{~s}, 1 \mathrm{H}, \mathrm{CH}), 3.34$ (s, 3H, OCH $\mathrm{OCH}_{3}, 2.42\left(\mathrm{~d}, \mathrm{~J}=13 \mathrm{~Hz}, 2 \mathrm{H}, \mathrm{CH}_{2}\right), 1.83\left(\mathrm{br}, 2 \mathrm{H}, \mathrm{CH}_{2}\right), 1.67-1.65\left(\mathrm{~m}, 5 \mathrm{H}, \mathrm{CH}_{2}\right), 1.34-129\left(\mathrm{~m}, 1 \mathrm{H}, \mathrm{CH}_{2}\right)$; ${ }^{13} \mathrm{C}$ NMR $\left(125 \mathrm{MHz}, \mathrm{CDCl}_{3}, 27{ }^{\circ} \mathrm{C}\right) \delta 168.4,165.1,147.3,138.8,137.8,136.6,134.6,130.6,129.3,128.7$, 128.0, 128.0, 127.5, 126.7, 125.6, 51.5, 43.2, 36.1, 26.1, 23.2; HRMS (ESI) m/z: $\left[M+\mathrm{H}^{+}\right.$calcd. for $\mathrm{C}_{29} \mathrm{H}_{30} \mathrm{NO}_{2}{ }^{+} 424.2271$ found 424.2270 .

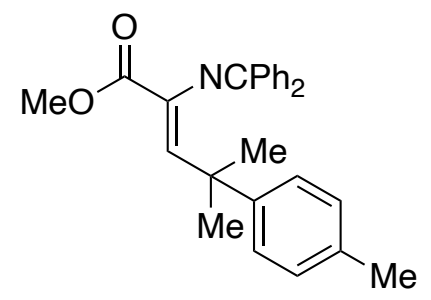

Methyl (Z)-2-((diphenylmethylene)amino)-4-methyl-4-(p-tolyl)pent-2-enoate (3al): (Pale yellow oil, $n$ Hexane/Diethyl ether = 20/1 to 4/1, General Procedure B: 19\% yield, 16.4 mg); IR (neat) 1716, 1622, 1444, 1240, $1080 \mathrm{~cm}^{-1} ;{ }^{1} \mathrm{H}$ NMR $\left(500 \mathrm{MHz}, \mathrm{CDCl}_{3}, 27^{\circ} \mathrm{C}\right) \delta 7.54(\mathrm{~d}, J=7.5 \mathrm{~Hz}, 2 \mathrm{H}, \operatorname{Ar} H), 7.42(\mathrm{t}, J=7.25 \mathrm{~Hz}, 1 \mathrm{H}$, $\operatorname{ArH}), 7.33(\mathrm{t}, J=7.5 \mathrm{~Hz}, 3 \mathrm{H}, \operatorname{ArH}), 7.28(\mathrm{t}, J=7.5 \mathrm{~Hz}, 2 \mathrm{H}, \operatorname{ArH}), 7.20(\mathrm{~d}, J=8.0 \mathrm{~Hz}, 2 \mathrm{H}, \operatorname{ArH}), 7.03(\mathrm{~d}, J=8.0 \mathrm{~Hz}$, $2 \mathrm{H}, \operatorname{ArH}), 6.92(\mathrm{~d}, J=7.0 \mathrm{~Hz}, 2 \mathrm{H}, \mathrm{ArH}), 6.30(\mathrm{~s}, 1 \mathrm{H}, \mathrm{CH}), 3.44\left(\mathrm{~s}, 3 \mathrm{H}, \mathrm{OCH}_{3}\right), 2.28\left(\mathrm{~s}, 3 \mathrm{H}, \mathrm{CH}_{3}\right), 1.54(\mathrm{~s}, 6 \mathrm{H}$, $\left.\mathrm{CH}_{3}\right) ;{ }^{13} \mathrm{C}$ NMR $\left(125 \mathrm{MHz}, \mathrm{CDCl}_{3}, 2{ }^{\circ} \mathrm{C}\right) \delta 168.5,165.4,145.6,138.8,137.3,136.8,135.2,135.1,130.6$, 129.4, 128.9, 128.7, 128.2, 127.9, 127.8, 126.1, 51.7, 39.6, 29.0, 20.9; HRMS (ESI) m/z: [M + H] ${ }^{+}$calcd. for $\mathrm{C}_{27} \mathrm{H}_{28} \mathrm{NO}_{2}{ }^{+} 398.2115$ found 398.2115 . 
<smiles>CC(/C=C(/C(=O)c1ccccc1)N(c1ccccc1)c1ccccc1)c1ccccc1</smiles>

Methyl (Z)-2-((diphenylmethylene)amino)-4-phenylpent-2-enoate (3am): (Pale yellow solid, $n$ Hexane/Dichloromethane/Diethyl ether = 20/10/1, General Procedure B: 61\% yield, $44.8 \mathrm{mg}$ ); IR (neat) 1708, 1637, 1290, 1234, 1136, $1066 \mathrm{~cm}^{-1} ;{ }^{1} \mathrm{H}$ NMR $\left(500 \mathrm{MHz}, \mathrm{CDCl}_{3}, 27^{\circ} \mathrm{C}\right) \delta 7.72(\mathrm{~d}, J=7.5 \mathrm{~Hz}, 2 \mathrm{H}, \operatorname{ArH})$, $7.48(\mathrm{t}, J=7.25 \mathrm{~Hz}, 1 \mathrm{H}, \operatorname{ArH}), 7.40(\mathrm{t}, J=7.5 \mathrm{~Hz}, 2 \mathrm{H}, \operatorname{ArH}), 7.32(\mathrm{t}, J=7.5 \mathrm{~Hz}, 1 \mathrm{H}, \operatorname{ArH}), 7.27-7.23(\mathrm{~m}, 4 \mathrm{H}$, $\operatorname{ArH}$ ), 7.19-7.14 (m, 3H, $\operatorname{ArH}), 6.97(\mathrm{~d}, J=7.0 \mathrm{~Hz}, 2 \mathrm{H}, \operatorname{ArH}), 6.16(\mathrm{~d}, J=10.0 \mathrm{~Hz}, 1 \mathrm{H}, \mathrm{CH}), 3.73$ (quartet of doublets, $J=10.0,7.0 \mathrm{~Hz}, 1 \mathrm{H}, \mathrm{CH}), 3.55\left(\mathrm{~s}, 3 \mathrm{H}, \mathrm{OCH}_{3}\right), 1.25$ (d, $\left.J=7.0 \mathrm{~Hz}, 3 \mathrm{H}, \mathrm{CH}_{3}\right) ;{ }^{13} \mathrm{C}$ NMR $(125 \mathrm{MHz}$, $\left.\mathrm{CDCl}_{3}, 27^{\circ} \mathrm{C}\right) \delta 170.8,164.9,144.6,138.9,138.5,136.6,130.9,130.2,129.5,129.1,128.5,128.2,128.1$, 127.9, 127.2, 126.2, 51.8, 37.3, 20.8; HRMS (ESI) m/z: $[M+H]^{+}$calcd. for $\mathrm{C}_{25} \mathrm{H}_{24} \mathrm{NO}_{2}{ }^{+} 370.1802$ found 370.1803 .

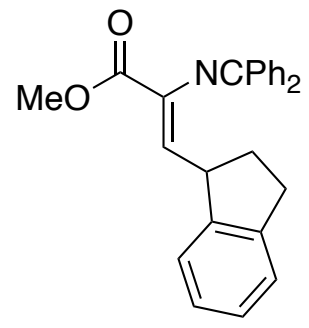

Methyl (Z)-3-(2,3-dihydro-1H-inden-1-yl)-2-((diphenylmethylene)amino)acrylate (3an): (Pale yellow solid, $n$-Hexane/Dichloromethane/Diethyl ether = 20/15/1, General Procedure B: 37\% yield, 28.3 mg); IR (neat) $1716,1635,1435,1274,1230 \mathrm{~cm}^{-1} ;{ }^{1} \mathrm{H} \mathrm{NMR}\left(500 \mathrm{MHz}, \mathrm{CDCl}_{3}, 27^{\circ} \mathrm{C}\right) \delta 7.75$ (d, J = $\left.7.5 \mathrm{~Hz}, 2 \mathrm{H}, \operatorname{ArH}\right)$, $7.46(\mathrm{t}, J=7.25 \mathrm{~Hz}, 1 \mathrm{H}, \operatorname{Ar} H), 7.43-7.37(\mathrm{~m}, 5 \mathrm{H}, \operatorname{Ar} H), 7.24-7.22(\mathrm{~m}, 2 \mathrm{H}, \operatorname{ArH}), 7.18(\mathrm{~d}, J=7.0 \mathrm{~Hz}, 1 \mathrm{H}, \operatorname{Ar} H)$, $7.11(\mathrm{dt}, J=21.5,7.25 \mathrm{~Hz}, 2 \mathrm{H}, \operatorname{ArH}), 6.87(\mathrm{~d}, J=7.0 \mathrm{~Hz}, 1 \mathrm{H}, \operatorname{ArH}), 6.02(\mathrm{~d}, J=10.0 \mathrm{~Hz}, 1 \mathrm{H}, \mathrm{CH}), 3.97(\mathrm{q}, J=$ $8.83 \mathrm{~Hz}, 1 \mathrm{H}, \mathrm{CH}), 3.65\left(\mathrm{~s}, 3 \mathrm{H}, \mathrm{OCH}_{3}\right), 2.90$ (ddd, $\left.J=15.5,8.5,3.5 \mathrm{~Hz}, 1 \mathrm{H}, \mathrm{CH}_{2}\right), 2.85-2.78\left(\mathrm{~m}, 2 \mathrm{H}, \mathrm{CH}_{2}\right), 2.16-$ $2.10(\mathrm{~m}, 1 \mathrm{H}, \mathrm{CH}), 1.68$ (quartet of doublets, $J=12.5,8.83 \mathrm{~Hz}, 1 \mathrm{H}, \mathrm{CH}) ;{ }^{13} \mathrm{C} \mathrm{NMR}\left(125 \mathrm{MHz}, \mathrm{CDCl}_{3}, 27{ }^{\circ} \mathrm{C}\right) \delta$ 171.0, 165.0, 145.2, 143.7, 140.1, 138.9, 136.7, 131.0, 129.5, 129.2, 128.4, 128.2, 127.6, 126.7, 126.3, 124.6, 124.3, 51.9, 43.3, 32.4, 31.8; HRMS (ESI) m/z: $[\mathrm{M}+\mathrm{H}]^{+}$calcd. for $\mathrm{C}_{26} \mathrm{H}_{24} \mathrm{NO}_{2}{ }^{+} 382.1802$ found 382.1802 . 
<smiles>COC(=O)/C(=C/C(c1ccccc1)c1ccccc1)c1ccccc1</smiles>

Methyl (Z)-2-((diphenylmethylene)amino)-4,4-diphenylbut-2-enoate (3ao): (Pale yellow solid, $n$ Hexane/Dichloromethane/Diethyl ether = 30/15/1, General Procedure B: 35\% yield, $30.4 \mathrm{mg}$ ); IR (neat) 1714, 1265, $1230 \mathrm{~cm}^{-1} ;{ }^{1} \mathrm{H}$ NMR $\left(500 \mathrm{MHz}, \mathrm{CDCl}_{3}, 27^{\circ} \mathrm{C}\right) \delta 7.70(\mathrm{~d}, J=7.0 \mathrm{~Hz}, 2 \mathrm{H}, \operatorname{ArH}), 7.47$ (t, J = $7.5 \mathrm{~Hz}$, $1 \mathrm{H}, \operatorname{ArH}), 7.39(\mathrm{t}, J=7.5 \mathrm{~Hz}, 2 \mathrm{H}, \operatorname{ArH}), 7.28-7.23(\mathrm{~m}, 5 \mathrm{H}, \operatorname{ArH}), 7.20-7.13(\mathrm{~m}, 4 \mathrm{H}, \operatorname{ArH}), 7.11(\mathrm{~d}, J=7.5 \mathrm{~Hz}$, $2 \mathrm{H}, \operatorname{ArH}), 6.82(\mathrm{~d}, J=7.0 \mathrm{~Hz}, 2 \mathrm{H}, \operatorname{ArH}), 6.56(\mathrm{~d}, J=10.0 \mathrm{~Hz}, 1 \mathrm{H}, \mathrm{CH}), 5.02(\mathrm{~d}, J=10.0 \mathrm{~Hz}, 1 \mathrm{H}, \mathrm{CH}), 3.55(\mathrm{~s}$, $\left.3 \mathrm{H}, \mathrm{OCH}_{3}\right) ;{ }^{13} \mathrm{C} \mathrm{NMR}\left(125 \mathrm{MHz}, \mathrm{CDCl}_{3}, 27^{\circ} \mathrm{C}\right) \delta 171.3,164.6,142.9,139.5,138.8,136.5,131.0,129.5,129.0$, 128.5, 128.1, 128.1, 127.8, 127.4, 126.4, 51.8, 48.3; HRMS (ESI) m/z: $[\mathrm{M}+\mathrm{H}]^{+}$calcd. for $\mathrm{C}_{30} \mathrm{H}_{26} \mathrm{NO}_{2}{ }^{+}$ 432.1958 found 432.1956 .<smiles>COC(=O)/C(=C/C1CC2CCC1C2)Nc1ccccc1</smiles>

Methyl (Z)-3-(bicyclo[2.2.1]heptan-2-yl)-2-((diphenylmethylene)amino)acrylate (3ap): (Pale yellow oil , $n$-Hexane/Dichloromethane/Diethyl ether = 20/4/4, General Procedure D: 38\% yield, $27.4 \mathrm{mg}$ )); IR (neat) 2947, 1716, 1633, 1446, 1236, $1068 \mathrm{~cm}^{-1} ;{ }^{1} \mathrm{H}$ NMR (500 MHz, CDCl, $\left.27^{\circ} \mathrm{C}\right) \delta 7.73$ (d, J = $\left.7.5 \mathrm{~Hz}, 2 \mathrm{H}, \operatorname{ArH}\right)$, 7.47 (triplet of triplets, $J=7.5,2.0,1.25 \mathrm{~Hz}, 1 \mathrm{H}, \mathrm{ArH}), 7.41-7.33(\mathrm{~m}, 5 \mathrm{H}, \mathrm{ArH}), 7.18-7.16(\mathrm{~m}, 2 \mathrm{H}, \mathrm{ArH}), 5.85$ $(\mathrm{d}, J=10.5 \mathrm{~Hz}, 1 \mathrm{H}, \mathrm{CH}), 3.64\left(\mathrm{~s}, 3 \mathrm{H}, \mathrm{OCH}_{3}\right), 3.34\left(\mathrm{~s}, 3 \mathrm{H}, \mathrm{OCH}_{3}\right), 2.20-2.16(\mathrm{~m}, 2 \mathrm{H}, \mathrm{CH}), 1.70(\mathrm{br}, 1 \mathrm{H}, \mathrm{CH})$, 1.44-1.37 (m, 4H, $\left.\left.\mathrm{CH}_{2}\right), 1.17-1.08\left(\mathrm{~m}, 4 \mathrm{H}, \mathrm{CH}_{2}\right) ;{ }^{13} \mathrm{C} \mathrm{NMR} \mathrm{(125} \mathrm{MHz,} \mathrm{CDCl} 3,27^{\circ} \mathrm{C}\right) \delta 170.7,165.3,139.0$, 137.1 , 136.7, 130.8, 129.5, 129.1, 128.3, 128.1, 128.0, 51.9, 41.9, 39.8, 37.7, 36.4, 36.0, 29.7, 28.8; HRMS (ESI) $\mathrm{m} / \mathrm{z}:[\mathrm{M}+\mathrm{H}]^{+}$calcd. for $\mathrm{C}_{24} \mathrm{H}_{26} \mathrm{NO}_{2}{ }^{+} 360.1958$ found 360.1957 . 


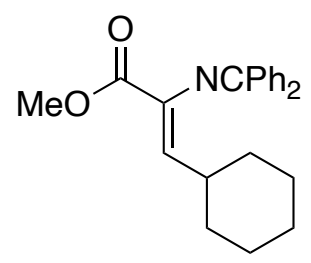

Methyl (Z)-3-cyclohexyl-2-((diphenylmethylene)amino)acrylate (3aq): (Pale yellow oil, $n$-Hexane/Diethyl ether = 10/1 to 6/1, General Procedure B: 45\% yield, $31.6 \mathrm{mg}$ ); IR (neat) 2924, 1716, 1444, 1251, 1220, $1064 \mathrm{~cm}^{-1} ;{ }^{1} \mathrm{H} \mathrm{NMR}\left(500 \mathrm{MHz}, \mathrm{CDCl}_{3}, 27^{\circ} \mathrm{C}\right) \delta 7.73(\mathrm{dt}, J=8.0,1.75,1.5 \mathrm{~Hz}, 2 \mathrm{H}, \mathrm{ArH}), 7.47$ (triplet of triplets, $J=7.25,2.25,1.25 \mathrm{~Hz}, 1 \mathrm{H}, \operatorname{ArH}), 7.41-7.33(\mathrm{~m}, 5 \mathrm{H}, \operatorname{ArH}), 7.19-7.17(\mathrm{~m}, 2 \mathrm{H}, \operatorname{ArH}), 5.81(\mathrm{~d}, J=10.0 \mathrm{~Hz}, 1 \mathrm{H}$, $\mathrm{CH}$ ), $3.62\left(\mathrm{~s}, 3 \mathrm{H}, \mathrm{OCH}_{3}\right), 2.14$ (quartet of triplets, $\left.J=10.0,3.75,3.5 \mathrm{~Hz}, 1 \mathrm{H}, \mathrm{CH}\right), 1.65-1.58\left(\mathrm{~m}, 3 \mathrm{H}, \mathrm{CH}_{2}\right), 1.37$ (d, $\left.J=13.5 \mathrm{~Hz}, 2 \mathrm{H}, \mathrm{CH}_{2}\right), 1.19-1.09\left(\mathrm{~m}, 3 \mathrm{H}, \mathrm{CH}_{2}\right), 1.03-0.96\left(\mathrm{~m}, 2 \mathrm{H}, \mathrm{CH}_{2}\right) ;{ }^{13} \mathrm{C} \mathrm{NMR}\left(125 \mathrm{MHz}, \mathrm{CDCl}_{3}, 27^{\circ} \mathrm{C}\right)$ $\delta 170.3,165.3,139.1,138.0,136.8,130.8,129.5,129.1,128.3,128.1,128.0,51.8,36.4,31.4,25.9$, 25.5; HRMS (ESI) m/z: [M + H] $]^{+}$calcd. for $\mathrm{C}_{23} \mathrm{H}_{26} \mathrm{NO}_{2}{ }^{+} 348.1958$ found 348.1956 .

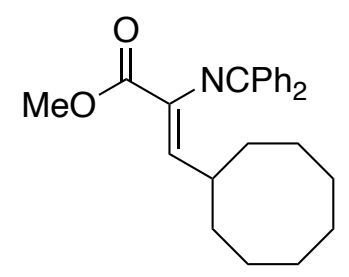

Methyl (Z)-3-cyclooctyl-2-((diphenylmethylene)amino)acrylate (3ar): (Pale yellow oil, $n$-Hexane/Diethyl ether $=10 / 1$ to 6/1, General Procedure B: 45\% yield, $33.9 \mathrm{mg}$ ); IR (neat) 2916, 1716, 1635, 1444, 1242, $1064 \mathrm{~cm}^{-1} ;{ }^{1} \mathrm{H}$ NMR $\left(500 \mathrm{MHz}, \mathrm{CDCl}_{3}, 27^{\circ} \mathrm{C}\right) \delta 7.72(\mathrm{~d}, J=7.0 \mathrm{~Hz}, 2 \mathrm{H}, \mathrm{ArH}), 7.47$ (triplet of triplets, $J=7.25$, 2.0, $1.25 \mathrm{~Hz}, 1 \mathrm{H}, \mathrm{ArH})$, 7.41-7.33 (m, 5H, ArH), 7.19-7.18 (m, 2H, ArH), $5.91(\mathrm{~d}, J=10.5 \mathrm{~Hz}, 1 \mathrm{H}, \mathrm{CH}), 3.64(\mathrm{~s}$, $\left.3 \mathrm{H}, \mathrm{OCH}_{3}\right), 2.39-2.32(\mathrm{~m}, 1 \mathrm{H}, \mathrm{CH}), 1.63-1.26\left(\mathrm{~m}, 14 \mathrm{H}, \mathrm{CH}_{2}\right) ;{ }^{13} \mathrm{C} \mathrm{NMR}\left(125 \mathrm{MHz}, \mathrm{CDCl}_{3}, 27^{\circ} \mathrm{C}\right) \delta 170.3,165.5$, 139.1, 137.0, 136.7, 131.7, 130.8, 129.4, 129.1, 128.3, 128.1, 128.0, 51.9, 35.8, 30.7, 27.2, 26.1, 25.1; HRMS (ESI) m/z: [M + H] $]^{+}$calcd. for $\mathrm{C}_{25} \mathrm{H}_{30} \mathrm{NO}_{2}{ }^{+} 376.2271$ found 376.2273 . 


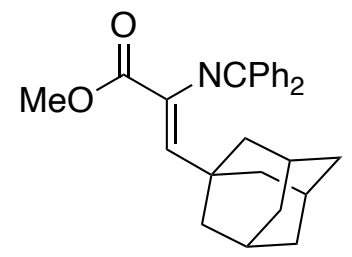

Methyl (Z)-3-((3r,5r,7r)-adamantan-1-yl)-2-((diphenylmethylene)amino)acrylate (3as): (Pale yellow solid, $n$-Hexane/Dichloromethane/Diethyl ether =60/40/1: 55\% yield, $44.0 \mathrm{mg}$ ); IR (neat) 2904, 1712, 1267, $1230 \mathrm{~cm}^{-1} ;{ }^{1} \mathrm{H}$ NMR $\left(500 \mathrm{MHz}, \mathrm{CDCl}_{3}, 27^{\circ} \mathrm{C}\right) \delta 7.76(\mathrm{~d}, J=7.0 \mathrm{~Hz}, 2 \mathrm{H}, \operatorname{ArH}), 7.46(\mathrm{t}, J=7.25 \mathrm{~Hz}, 1 \mathrm{H}$, $\operatorname{ArH}), 7.41-7.37(\mathrm{~m}, 5 \mathrm{H}, \mathrm{ArH}), 7.24-7.22(\mathrm{~m}, 2 \mathrm{H}, \mathrm{ArH}), 5.83(\mathrm{~s}, 1 \mathrm{H}, \mathrm{CH}), 3.44(\mathrm{~s}, 3 \mathrm{H}, \mathrm{OCH}$ ), $1.94(\mathrm{br}, 3 \mathrm{H}, \mathrm{CH})$, $1.86\left(\mathrm{~d}, J=2.5 \mathrm{~Hz}, 6 \mathrm{H}, \mathrm{CH}_{2}\right), 1.66\left(\mathrm{br}, 6 \mathrm{H}, \mathrm{CH}_{2}\right) ;{ }^{13} \mathrm{C} \mathrm{NMR}\left(125 \mathrm{MHz}, \mathrm{CDCl}_{3}, 27{ }^{\circ} \mathrm{C}\right) \delta 168.5,165.7,139.0$, 137.0, 136.9, 136.3, 130.7, 129.4, 128.9, 128.5, 128.1, 127.9, 51.6, 41.4, 36.7, 35.4, 28.4; HRMS (ESI) m/z: $[\mathrm{M}+\mathrm{H}]^{+}$calcd. for $\mathrm{C}_{27} \mathrm{H}_{30} \mathrm{NO}_{2}{ }^{+} 400.2271$ found 400.2273 .

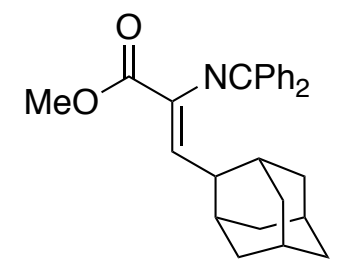

Methyl (Z)-3-((1r,3r,5r,7r)-adamantan-2-yl)-2-((diphenylmethylene)amino)acrylate (3as'): (Pale yellow solid, $n$-Hexane/Dichloromethane/Diethyl ether =60/40/1: 19\% yield, $15.3 \mathrm{mg}$ ); IR (neat) 2904, 1712, 1265, $1228 \mathrm{~cm}^{-1} ;{ }^{1} \mathrm{H}$ NMR $\left(500 \mathrm{MHz}, \mathrm{CDCl}_{3}, 27^{\circ} \mathrm{C}\right) \delta 7.71(\mathrm{~d}, J=7.0 \mathrm{~Hz}, 2 \mathrm{H}, \mathrm{ArH}), 7.46(\mathrm{t}, J=7.5 \mathrm{~Hz}, 1 \mathrm{H}$, $\operatorname{ArH}), 7.40-7.32(\mathrm{~m}, 5 \mathrm{H}, \mathrm{ArH}), 7.18-7.16(\mathrm{~m}, 2 \mathrm{H}, \mathrm{ArH}), 6.35(\mathrm{~d}, J=10.0 \mathrm{~Hz}, 1 \mathrm{H}, \mathrm{CH}), 3.63(\mathrm{~s}, 3 \mathrm{H}, \mathrm{OCH}), 2.54$ $(\mathrm{d}, J=10.0 \mathrm{~Hz}, 2 \mathrm{H}, \mathrm{CH}), 1.94\left(\mathrm{~d}, J=12.5 \mathrm{~Hz}, 2 \mathrm{H}, \mathrm{CH}_{2}\right), 1.82\left(\mathrm{br}, 2 \mathrm{H}, \mathrm{CH}_{2}\right), 1.77\left(\mathrm{~d}, J=12.5 \mathrm{~Hz}, 2 \mathrm{H}, \mathrm{CH}_{2}\right), 1.70-$ $1.66\left(\mathrm{~m}, 4 \mathrm{H}, \mathrm{CH}_{2}\right), 1.59(\mathrm{br}, 2 \mathrm{H}, \mathrm{CH}), 1.52\left(\mathrm{br}, 2 \mathrm{H}, \mathrm{CH}_{2}\right) ;{ }^{13} \mathrm{C} \mathrm{NMR}\left(125 \mathrm{MHz}, \mathrm{CDCl}_{3}, 27^{\circ} \mathrm{C}\right) \delta 170.1,165.2$, 139.0, 138.6, 136.9, 130.8, 129.4, 129.1, 128.8, 128.1, 128.0, 51.8, 42.7, 38.5, 37.9, 32.4, 31.3, 27.8, 27.6; HRMS (ESI) m/z: [M+ H] calcd. for $\mathrm{C}_{27} \mathrm{H}_{30} \mathrm{NO}_{2}{ }^{+} 400.2271$ found 400.2272 . 


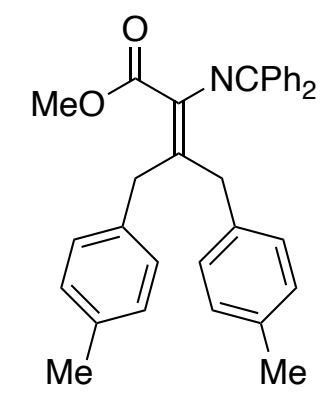

Methyl 2-((diphenylmethylene)amino)-3-(4-methylbenzyl)-4-(p-tolyl)but-2-enoate (3at): (Pale yellow solid, $n$-Hexane/Dichloromethane/Diethyl ether = 30/10/1, General Procedure B: 36\% yield, 33.9 mg); IR (neat) $1718,1508,1219 \mathrm{~cm}^{-1} ;{ }^{1} \mathrm{H}$ NMR $\left(500 \mathrm{MHz}, \mathrm{CDCl}_{3}, 27^{\circ} \mathrm{C}\right) \delta 7.76$ (d, J = 7.0 Hz, 2H, ArH), 7.47-7.35 $(\mathrm{m}, 6 \mathrm{H}, \operatorname{ArH}), 7.12-7.06(\mathrm{~m}, 6 \mathrm{H}, \operatorname{ArH}), 7.20(\mathrm{~d}, J=8.0 \mathrm{~Hz}, 2 \mathrm{H}, \operatorname{ArH}), 7.00(\mathrm{~d}, J=7.5 \mathrm{~Hz}, 2 \mathrm{H}, \operatorname{ArH}), 6.81(\mathrm{~d}, J=$ $7.5 \mathrm{~Hz}, 2 \mathrm{H}, \mathrm{ArH}), 3.63\left(\mathrm{~s}, 2 \mathrm{H}, \mathrm{CH}_{2}\right), 3.48\left(\mathrm{~s}, 3 \mathrm{H}, \mathrm{OCH}_{3}\right), 3.36\left(\mathrm{~s}, 2 \mathrm{H}, \mathrm{CH}_{2}\right), 2.32\left(\mathrm{~s}, 6 \mathrm{H}, \mathrm{CH}_{3}\right) ;{ }^{13} \mathrm{C}$ NMR (125 $\left.\mathrm{MHz}, \mathrm{CDCl}_{3}, 27^{\circ} \mathrm{C}\right) \delta 169.9,165.5,138.9,137.3,137.1,137.0,136.5,136.2,135.6,135.3,130.8,129.3$, 129.3, 129.1, 129.0, 129.0, 128.9, 128.2, 128.2, 128.1, 51.4, 36.4, 35.8, 21.1; HRMS (ESI) m/z: [M + H] calcd. for $\mathrm{C}_{33} \mathrm{H}_{32} \mathrm{NO}_{2}{ }^{+} 474.2428$ found 474.2426 .<smiles>CCCCCCCCN(CC)/C(C(=O)OC)=C(/C)Cc1ccc(C)cc1</smiles>

Methyl (Z)-2-((diphenylmethylene)amino)-3-methyl-4-(p-tolyl)but-2-enoate (3bt): (Pale yellow oil, $n$ Hexane/Dichloromethane/Diethyl ether = 20/15/1, General Procedure B: 28\% yield, $21.4 \mathrm{mg}$ ); IR (neat) 1714, 1510, 1444, 1234, $1058 \mathrm{~cm}^{-1}$; ${ }^{1} \mathrm{H}$ NMR (500 MHz, CDCl $\left.3,2{ }^{\circ} \mathrm{C}\right) \delta 7.74$ (d, J = 7.0 Hz, 2H, ArH), 7.47$7.43(\mathrm{~m}, 2 \mathrm{H}, \operatorname{ArH}), 7.38(\mathrm{q}, J=7.17 \mathrm{~Hz}, 4 \mathrm{H}, \operatorname{ArH}), 7.13(\mathrm{dd}, J=8.25,1.25 \mathrm{~Hz}, 2 \mathrm{H}, \operatorname{ArH}), 6.99(\mathrm{~d}, J=8.0 \mathrm{~Hz}$, $2 \mathrm{H}, \operatorname{ArH}), 6.82(\mathrm{~d}, J=8.0 \mathrm{~Hz}, 2 \mathrm{H}, \mathrm{ArH}), 3.74\left(\mathrm{~s}, 2 \mathrm{H}, \mathrm{CH}_{2}\right), 3.57\left(\mathrm{~s}, 3 \mathrm{H}, \mathrm{OCH}_{3}\right), 2.30\left(\mathrm{~s}, 3 \mathrm{H}, \mathrm{CH}_{3}\right), 1.62(\mathrm{~s}, 3 \mathrm{H}$, $\left.\mathrm{CH}_{3}\right) ;{ }^{13} \mathrm{C}$ NMR $\left(125 \mathrm{MHz}, \mathrm{CDCl}_{3}, 2{ }^{\circ} \mathrm{C}\right) \delta 170.2,165.1,138.9,137.3,136.5,136.2,135.3,134.1,130.8$, 129.2, 129.0, 128.9, 128.7, 128.2, 128.2, 127.9, 51.4, 39.2, 21.0, 19.3; HRMS (ESI) m/z: [M + H] calcd. for $\mathrm{C}_{26} \mathrm{H}_{26} \mathrm{NO}_{2}{ }^{+} 384.1958$ found 384.1956 . 
<smiles>COC(=O)C(=C(Cc1ccc(C)cc1)N(c1ccccc1)c1ccccc1)c1ccccc1</smiles>

methyl (E)-2-((diphenylmethylene)amino)-3-phenyl-4-(p-tolyl)but-2-enoate (3ct): (Pale yellow oil, $n$ Hexane/Diethyl ether/Methanol = 30/3/1, General Procedure B: 21\% yield, 19.7 mg); IR (neat) 1716, 1444, 1236, $1103 \mathrm{~cm}^{-1} ;{ }^{1} \mathrm{H}$ NMR $\left(500 \mathrm{MHz}, \mathrm{CDCl}_{3}, 27^{\circ} \mathrm{C}\right) \delta 7.51(\mathrm{~d}, J=7.0 \mathrm{~Hz}, 2 \mathrm{H}, \operatorname{ArH}), 7.42-7.37(\mathrm{~m}, 2 \mathrm{H}, \operatorname{ArH})$, $7.32-7.28(\mathrm{~m}, 4 \mathrm{H}, \operatorname{ArH}), 7.11(\mathrm{t}, J=7.5 \mathrm{~Hz}, 2 \mathrm{H}, \operatorname{ArH}), 7.05(\mathrm{t}, J=7.5 \mathrm{~Hz}, 1 \mathrm{H}, \operatorname{ArH}), 6.98(\mathrm{~d}, J=7.0 \mathrm{~Hz}, 2 \mathrm{H}$, $\operatorname{ArH}), 6.92(\mathrm{~d}, J=8.0 \mathrm{~Hz}, 2 \mathrm{H}, \mathrm{ArH}), 6.83(\mathrm{~d}, J=7.0 \mathrm{~Hz}, 2 \mathrm{H}, \mathrm{ArH}), 6.80(\mathrm{~d}, J=8.0 \mathrm{~Hz}, 2 \mathrm{H}, \operatorname{ArH}), 3.96(\mathrm{~s}, 2 \mathrm{H}$, $\mathrm{CH} 2), 3.69\left(\mathrm{~s}, 3 \mathrm{H}, \mathrm{OCH}_{3}\right), 2.25\left(\mathrm{~s}, 3 \mathrm{H}, \mathrm{CH}_{3}\right) ;{ }^{13} \mathrm{C} \mathrm{NMR}\left(125 \mathrm{MHz}, \mathrm{CDCl}_{3}, 27^{\circ} \mathrm{C}\right) \delta 169.7,166.0,140.2,138.9$, $138.1,137.1,136.4,135.0,132.3,130.6,129.3,129.0,128.9,128.7,128.5,128.2$, 128.0, 127.9, 127.5, 126.8, 51.9, 38.9, 21.0; HRMS (ESI) m/z: [M + H] $]^{+}$calcd. for $\mathrm{C}_{31} \mathrm{H}_{28} \mathrm{NO}_{2}{ }^{+} 446.2115$ found 446.2113 .<smiles>COC(=O)/C(Nc1ccccc1)=C(\C)C(C)c1ccccc1</smiles>

Methyl (Z)-2-((diphenylmethylene)amino)-3-methyl-4-phenylpent-2-enoate (3bm): (Pale yellow oil, $n$ Hexane/Dichloromethane/Diethyl ether = 15/5/1, General Procedure B: 18\% yield, $14.1 \mathrm{mg}$ ); IR (neat) 1712, 1444, 1259, 1215, $1074 \mathrm{~cm}^{-1}$; ${ }^{1} \mathrm{H}$ NMR (500 MHz, CDCl, $27^{\circ} \mathrm{C}$ ) $\delta 7.73$ (d, J = 7.0 Hz, 2H, ArH), 7.46$7.42(\mathrm{~m}, 2 \mathrm{H}, \operatorname{ArH}), 7.38(\mathrm{q}, J=7.5 \mathrm{~Hz}, 4 \mathrm{H}, \operatorname{ArH}), 7.20(\mathrm{t}, J=7.25 \mathrm{~Hz}, 2 \mathrm{H}, \operatorname{ArH}), 7.16-7.13(\mathrm{~m}, 3 \mathrm{H}, \operatorname{ArH}), 7.03$ $(\mathrm{d}, J=8.0 \mathrm{~Hz}, 2 \mathrm{H}, \mathrm{ArH}), 4.90(\mathrm{q}, J=7.0 \mathrm{~Hz}, 1 \mathrm{H}, \mathrm{CH}), 3.62\left(\mathrm{~s}, 3 \mathrm{H}, \mathrm{OCH}_{3}\right), 1.38\left(\mathrm{~s}, 3 \mathrm{H}, \mathrm{CH}_{3}\right), 1.33(\mathrm{~d}, J=7.0 \mathrm{~Hz}$, $\left.3 \mathrm{H}, \mathrm{CH}_{3}\right) ;{ }^{13} \mathrm{C} \mathrm{NMR}\left(125 \mathrm{MHz}, \mathrm{CDCl}_{3}, 27^{\circ} \mathrm{C}\right) \delta 170.2,165.2,143.5,138.9,138.2,137.3,135.4,130.8,129.2$, 129.0, 128.2, 128.1, 128.0, 127.9, 127.5, 125.8, 51.5, 38.5, 16.4, 14.4; HRMS (ESI) m/z: [M + H] ${ }^{+}$calcd. for $\mathrm{C}_{26} \mathrm{H}_{26} \mathrm{NO}_{2}{ }^{+} 384.1958$ found 384.1959 . 
<smiles>COC(=O)C(=CC(C(=O)OC)C(C)(C)c1ccccc1)C(=O)OC</smiles>

Dimethyl (Z)-2-((diphenylmethylene)amino)-4-(2-phenylpropan-2-yl)pent-2-enedioate (3da): (Pale yellow oil, $n$-Hexane/Diethyl ether = 10/3 $\left(1 \% \mathrm{Et}_{3} \mathrm{~N}\right)$, General Procedure B: $33 \%$ yield, $\left.30.3 \mathrm{mg}\right)$; IR (neat) 1720, 1433, 1244, 1139, $1066 \mathrm{~cm}^{-1} ;{ }^{1} \mathrm{H}$ NMR $\left(500 \mathrm{MHz}, \mathrm{CDCl}_{3}, 27^{\circ} \mathrm{C}\right) \delta 7.71$ (d, J = 7.0 Hz, 2H, ArH), $7.49(\mathrm{t}$, $J=7.25 \mathrm{~Hz}, 1 \mathrm{H}, \operatorname{ArH}), 7.41(\mathrm{t}, J=7.75 \mathrm{~Hz}, 2 \mathrm{H}, \operatorname{ArH}), 7.34-7.23(\mathrm{~m}, 8 \mathrm{H}, \operatorname{ArH}), 7.16(\mathrm{t}, J=7.25 \mathrm{~Hz}, 1 \mathrm{H}, \operatorname{ArH})$, $7.03(\mathrm{~d}, J=7.0 \mathrm{~Hz}, 2 \mathrm{H}, \mathrm{ArH}), 6.11(\mathrm{~d}, J=10.0 \mathrm{~Hz}, 1 \mathrm{H}, \mathrm{CH}), 3.73(\mathrm{~d}, J=10.0 \mathrm{~Hz}, 1 \mathrm{H}, \mathrm{CH}), 3.50\left(\mathrm{~s}, 3 \mathrm{H}, \mathrm{OCH}_{3}\right)$, $3.35\left(\mathrm{~s}, 3 \mathrm{H}, \mathrm{OCH}_{3}\right), 1.46\left(\mathrm{~d}, J=12 \mathrm{~Hz}, 6 \mathrm{H}, \mathrm{CH}_{3}\right) ;{ }^{13} \mathrm{C} \mathrm{NMR}\left(125 \mathrm{MHz}, \mathrm{CDCl}_{3}, 27^{\circ} \mathrm{C}\right) \delta 171.7,170.9,164.2$, 146.8, 141.6, 138.7, 136.5, 131.0, 129.6, 129.1, 128.3, 128.1, 128.0, 127.9, 126.2, 126.2, 121.2, 54.6, 51.8, 51.2, 41.8, 26.4, 25.9; HRMS (ESI) m/z: [M + H] ${ }^{+}$calcd. for $\mathrm{C}_{29} \mathrm{H}_{30} \mathrm{NO}_{4}{ }^{+} 456.2169$ found 456.2169 . 


\section{Transformation of the Products}<smiles>COC(=O)C(N)CC(C)(C)c1ccccc1</smiles>

Methyl 2-amino-4-methyl-4-phenylpentanoate (4); CAS Registry Number 2352034-33-2:

$50 \mathrm{~mL}$ round-bottom flask was charged with 3 aa $(0.40 \mathrm{mmol}, 1.0$ equiv.) and EtOH $(7.0 \mathrm{~mL})$, followed by $10 \% \mathrm{Pd} / \mathrm{C}(0.020 \mathrm{mmol}, 10 \mathrm{~mol} \%)$. After filling the flask with hydrogen, the solution was stirred for 6 hours at ambient temperature. Then, the reaction mixture was filtered and purified by flash column chromatography ( $n$-Hexane/Ethyl acetate $=1 / 1\left(1 \% \mathrm{Et}_{3} \mathrm{~N}\right), 80 \%$ yield, colorless liquid, $\left.70.5 \mathrm{mg}\right)$; IR (neat) 1732, 1496, 1436, 1195, 1168, $1029 \mathrm{~cm}^{-1} ;{ }^{1} \mathrm{H} \mathrm{NMR}\left(500 \mathrm{MHz}, \mathrm{CDCl}_{3}, 27^{\circ} \mathrm{C}\right) \delta 7.36$ (dd, J = 8.5, $1.0 \mathrm{~Hz}, 2 \mathrm{H}$, $\operatorname{ArH}), 7.31(\mathrm{t}, J=7.75 \mathrm{~Hz}, 2 \mathrm{H}, \operatorname{Ar} H), 7.18$ (triplet of triplets, $J=7.25,1.5,1.25 \mathrm{~Hz}, 1 \mathrm{H}, \operatorname{ArH}), 3.50(\mathrm{~s}, 3 \mathrm{H}$, $\left.\mathrm{OCH}_{3}\right), 3.32(\mathrm{dd}, J=7.0,6.0 \mathrm{~Hz}, 1 \mathrm{H}, \mathrm{CH}), 2.23(\mathrm{dd}, J=14,6.0 \mathrm{~Hz}, 1 \mathrm{H}, \mathrm{CH}), 1.83(\mathrm{dd}, J=14,7.0 \mathrm{~Hz}, 3 \mathrm{H}, \mathrm{CH}$ ), $1.41\left(\mathrm{~s}, 3 \mathrm{H}, \mathrm{CH}_{3}\right), 1.35$ (s, 3H, $\left.\mathrm{CH}_{3}\right) ;{ }^{13} \mathrm{C} \mathrm{NMR}\left(125 \mathrm{MHz}, \mathrm{CDCl}_{3}, 27^{\circ} \mathrm{C}\right) \delta 176.6,148.0,128.2,126.0,125.9$, 52.3, 51.8, 49.4, 37.3, 29.5, 29.3; HRMS (ESI) m/z: [M + H] calcd. for $\mathrm{C}_{13} \mathrm{H}_{20} \mathrm{NO}_{2}{ }^{+} 222.1489$ found 222.1488. 


\section{References}

1. O'Donnell, M. J.; Polt, R. L. A Mild and Efficient Route to Schiff Base Derivatives of Amino Acids. J. Org. Chem. 1982, 47, 2663-2666. 

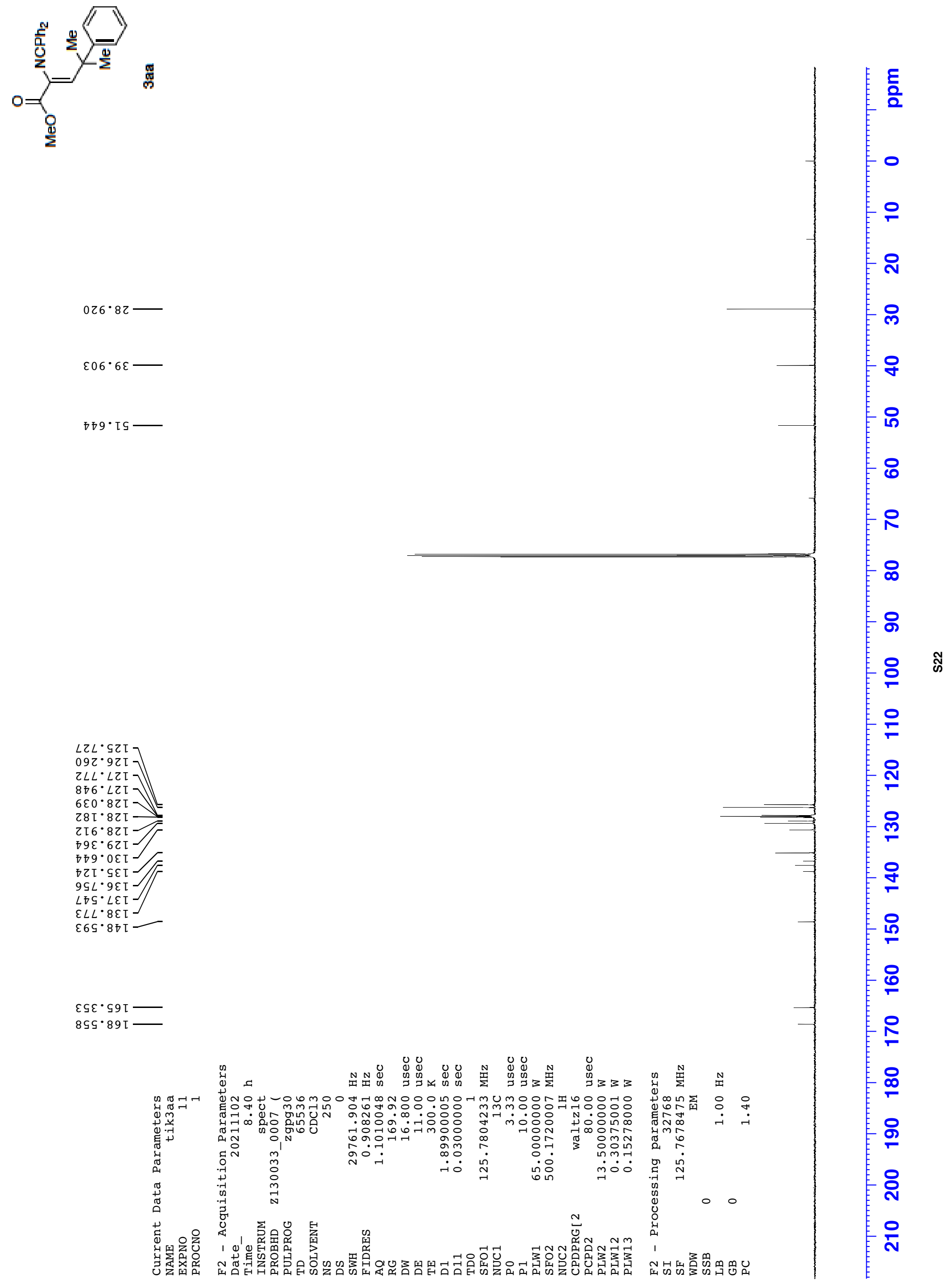


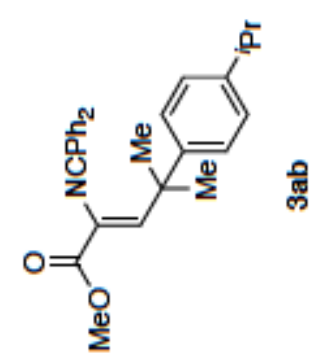

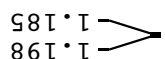

S9 $\mathrm{S}^{\circ} \mathrm{T}$

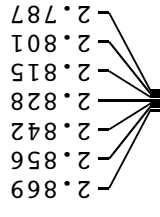

$\angle I \nabla \cdot \varepsilon-$

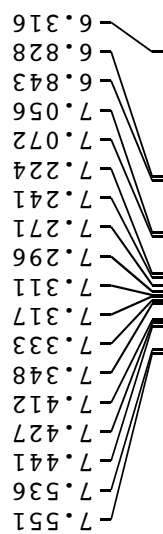

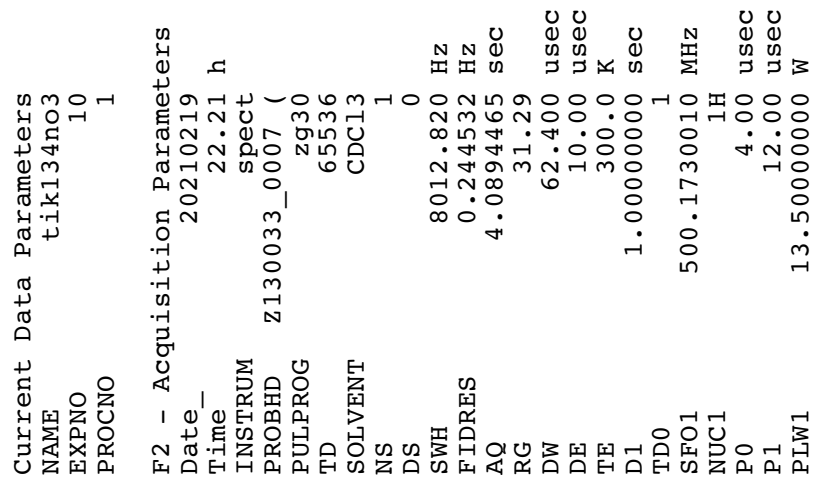

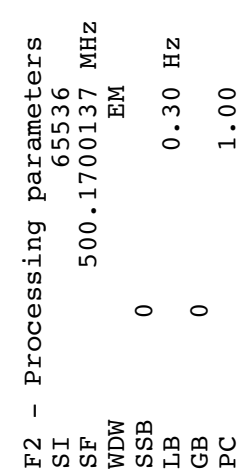

OZL'S

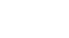




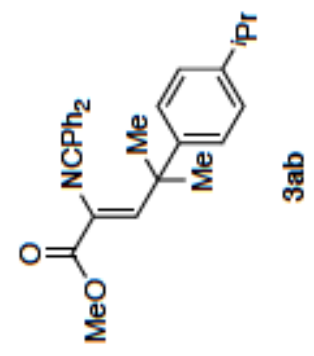

てゅ0・カて

$298 \cdot 82$

โ9 $9 \cdot \varepsilon \varepsilon$

$6 \mathrm{IS} \cdot 6 \varepsilon-$

วโ9・ โร

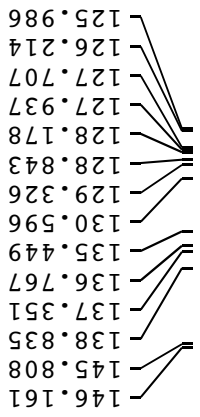

$6 \succsim \varepsilon \cdot \varsigma 9 \tau$

$70 \mathrm{~S}^{\cdot} 89 \mathrm{I}$

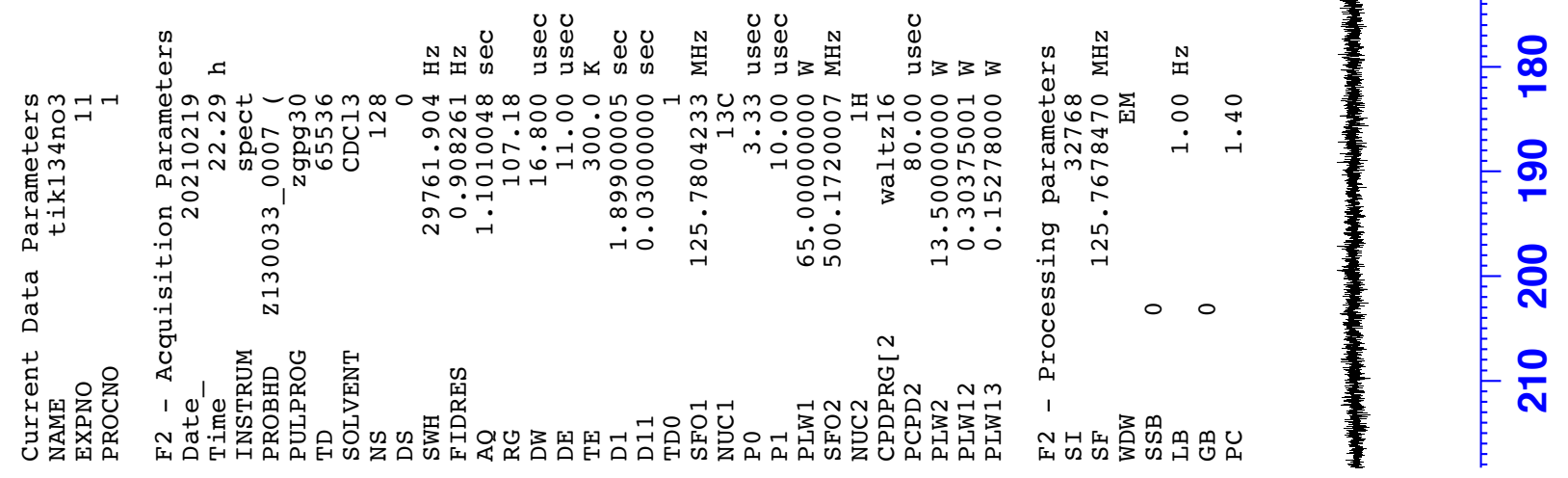




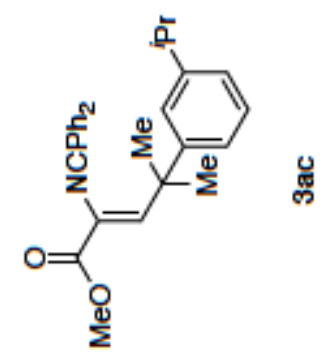

$\mathrm{SZI} \cdot \mathrm{I} \longrightarrow$
$6 \varepsilon \mathrm{I} \cdot \mathrm{I}$

$\angle 6 G^{\circ} \mathrm{I}$

$8[L \cdot Z$

$Z \varepsilon L \cdot Z$

$09 L \cdot Z$

$\nabla L L \cdot Z$

$88 \mathrm{~L} \cdot \mathrm{Z}$

$208^{\circ} \mathrm{Z}$

$20 \sigma^{\circ} \varepsilon$

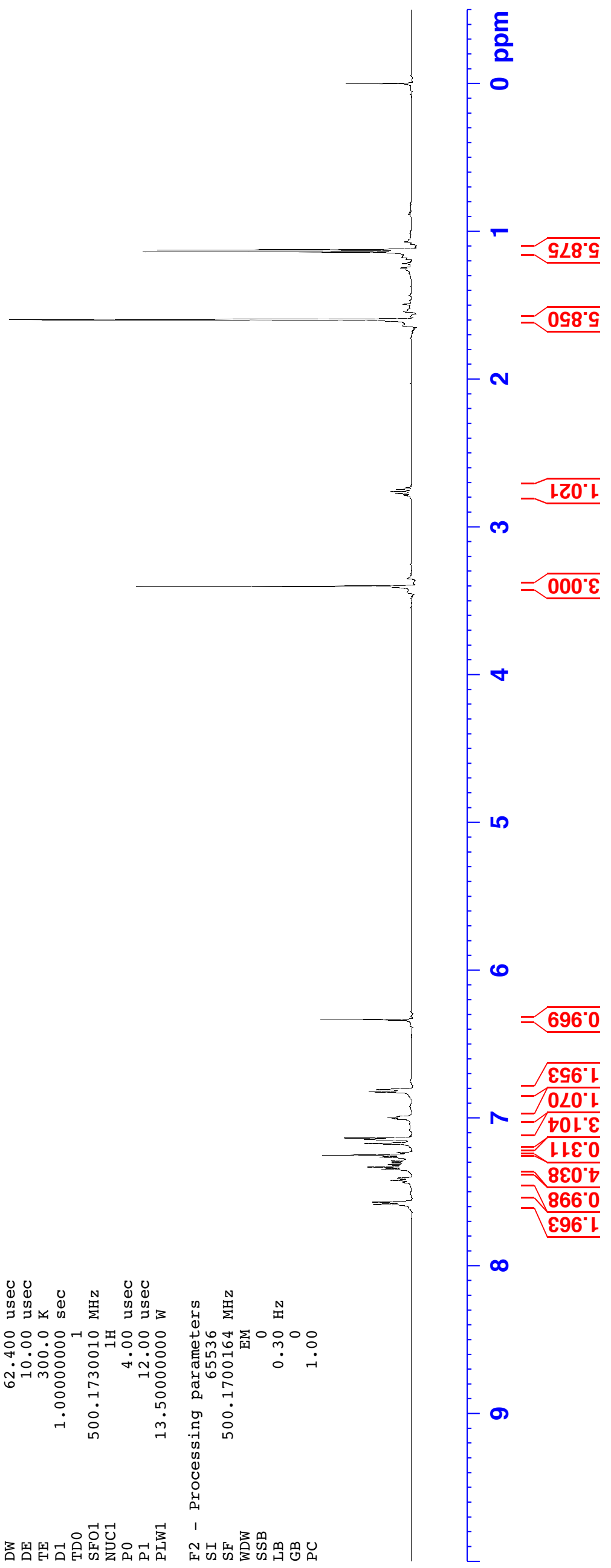

ฆึ

ธع $\cdot 9$

$808 \cdot 9$

$\varepsilon 乙 8^{\circ} 9$

$\left.\begin{array}{l}886^{\circ} \cdot 9 \\ 266 \cdot 9\end{array}\right]$

$\left.666^{\circ} 9\right]$

$\left.500^{\circ} L\right]$

$\left.600^{\circ} L\right]$

$L E[\cdot L]$

S†T $L$

ZLI $L$

G $9 Z^{\circ} L$

$06 Z^{\circ} L$

ฤ $0 \varepsilon \cdot L-$

$L[\varepsilon \cdot L J$

$\angle \nabla \varepsilon \cdot L]$

$800^{\circ} L^{\circ}$

ZZ๐ $L E{ }^{\circ} L J$

$69 g^{\circ} L$

S $89^{\circ} L$

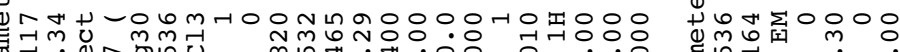

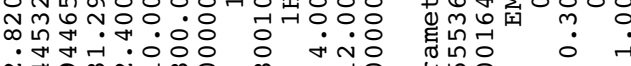

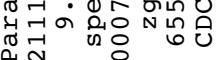




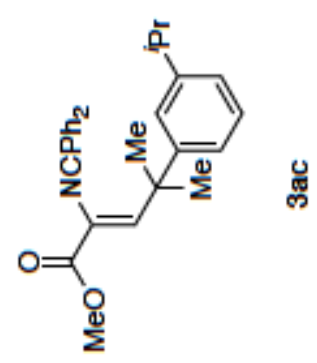

โย $0^{\bullet}$ Ð

$\varepsilon L L \cdot 8 Z$

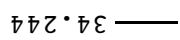

$26 L \cdot 6 \varepsilon$

$089^{\circ}$ IS

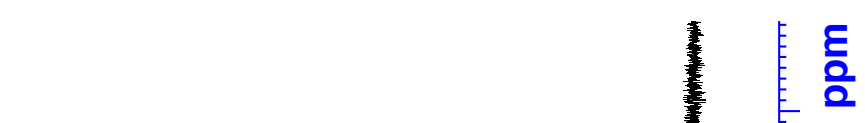

등

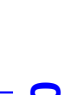

SOS. $\varepsilon Z \mathrm{~T}$

$0<\div \cdot \nabla Z T$

$Z T L \cdot \angle Z T$

T96. LZT

$9 L \mathrm{~T} \cdot 8 \mathrm{LT}$

598.8ZT

$\angle \mathrm{LE} \cdot 6 \mathrm{TL}$

$629 \cdot 0 \varepsilon \mathrm{T}$

089.

$\angle 9 L \cdot 9 \varepsilon \mathrm{L}$

9 โ $\varsigma^{\circ} \angle \varepsilon[-$

$\varepsilon L L \cdot 8 \varepsilon[-$

$907 \cdot 8$ 万

$76 \nabla \cdot 8 \nabla$ 


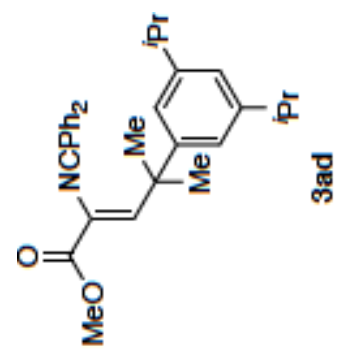

OธL $[\longrightarrow$

$ธ \mathrm{~S}^{\cdot} \mathrm{T}$

$8 \mathrm{I9^{ \circ }} \mathrm{T}$

$Z 0 L \cdot Z$

$9 \mathrm{~L} L$
$6 Z L^{\circ} Z$

$\varepsilon \nabla L \cdot \tau$

$\angle S L \cdot Z$

I $L L^{\circ} Z$

$\varsigma 8 L \cdot \tau]$

乙8⿷

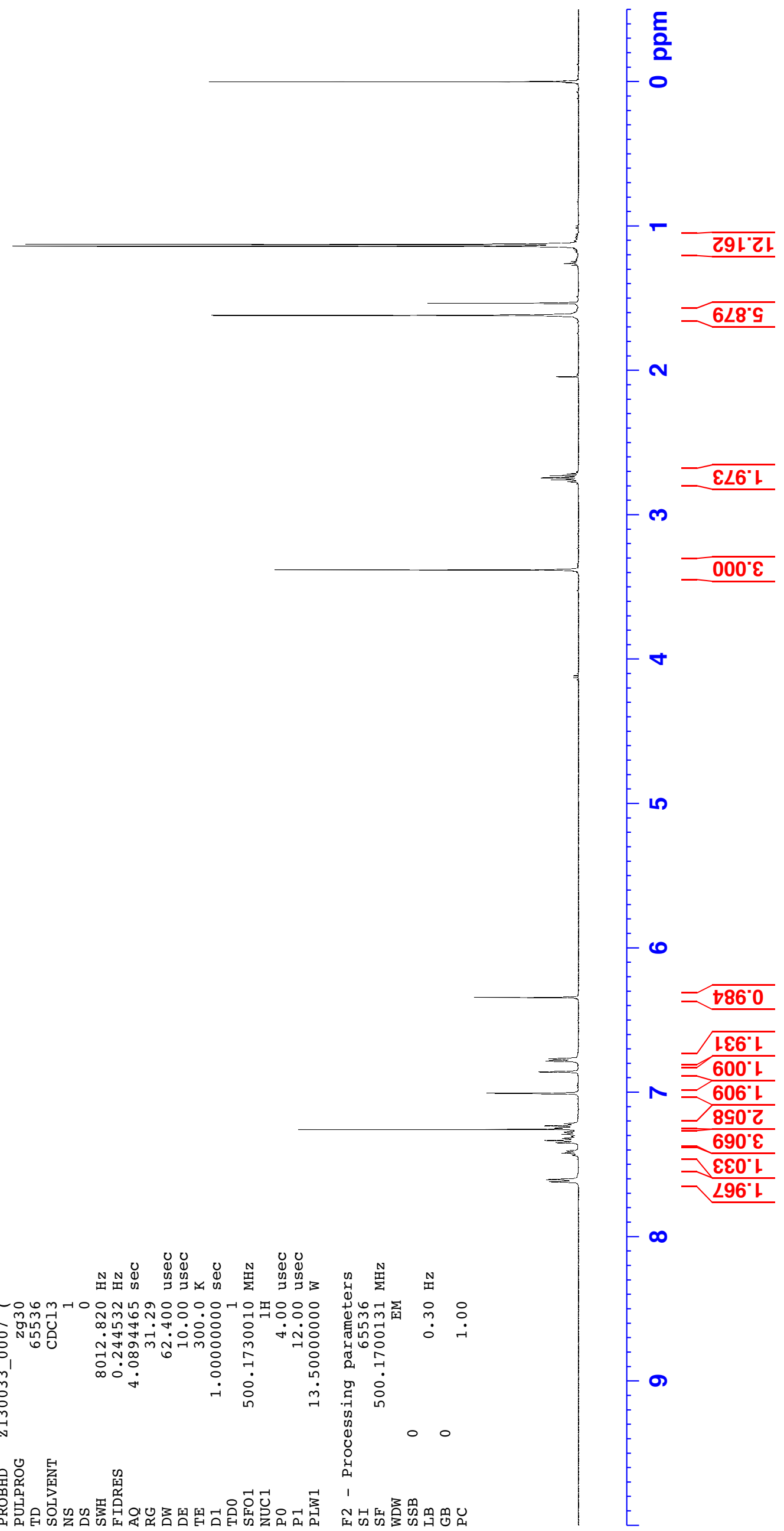

ธิ

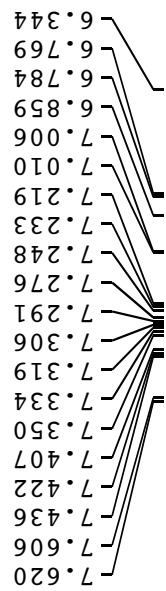

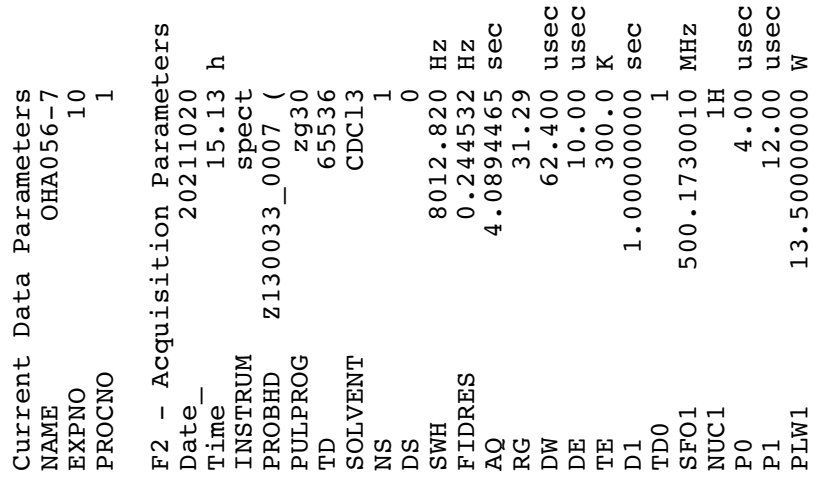




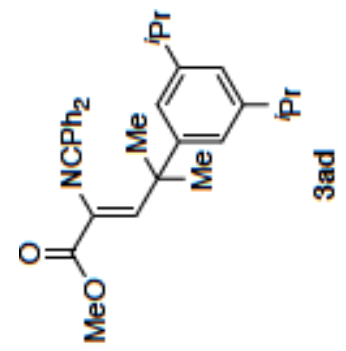

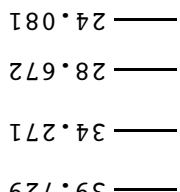

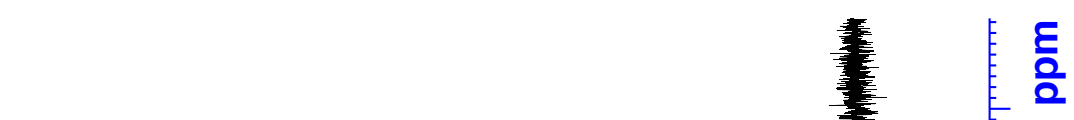

$6 Z L \cdot 6 \varepsilon$

$80 S^{*}$ โ

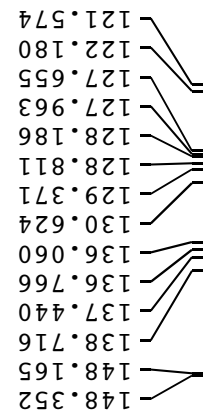

$97 \varepsilon \cdot \varsigma 9$ ᄂ

$\angle Z \varepsilon^{\circ} 89 \mathrm{I}$

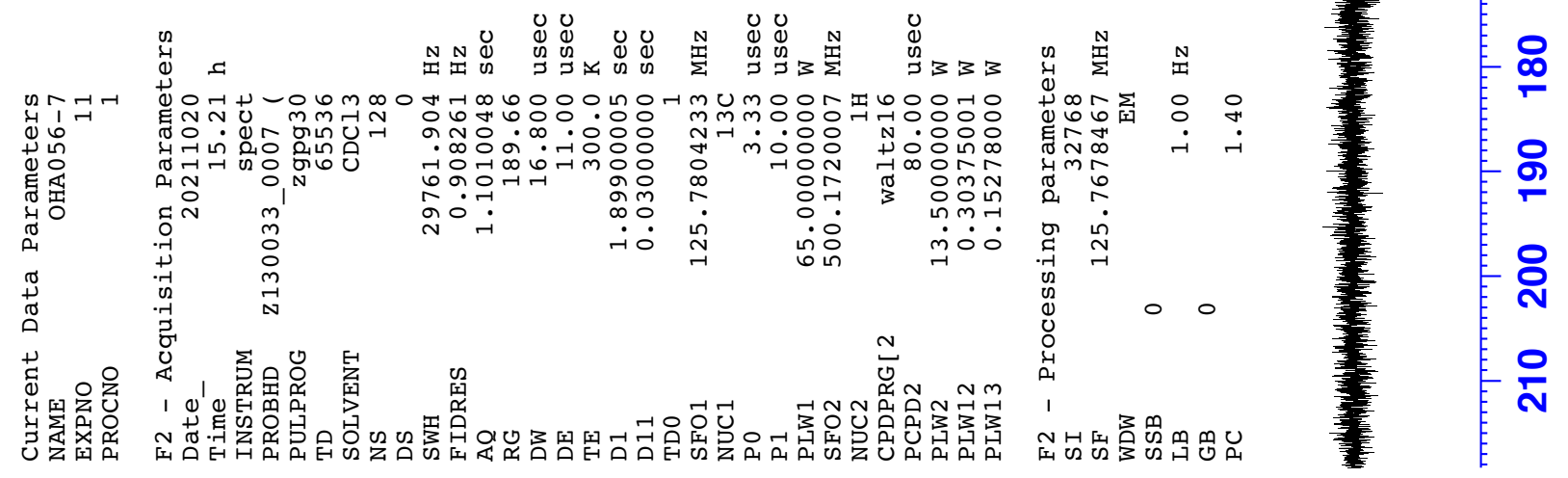




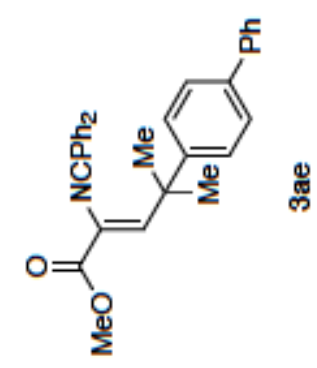

$76 S^{\circ} \mathrm{I}$

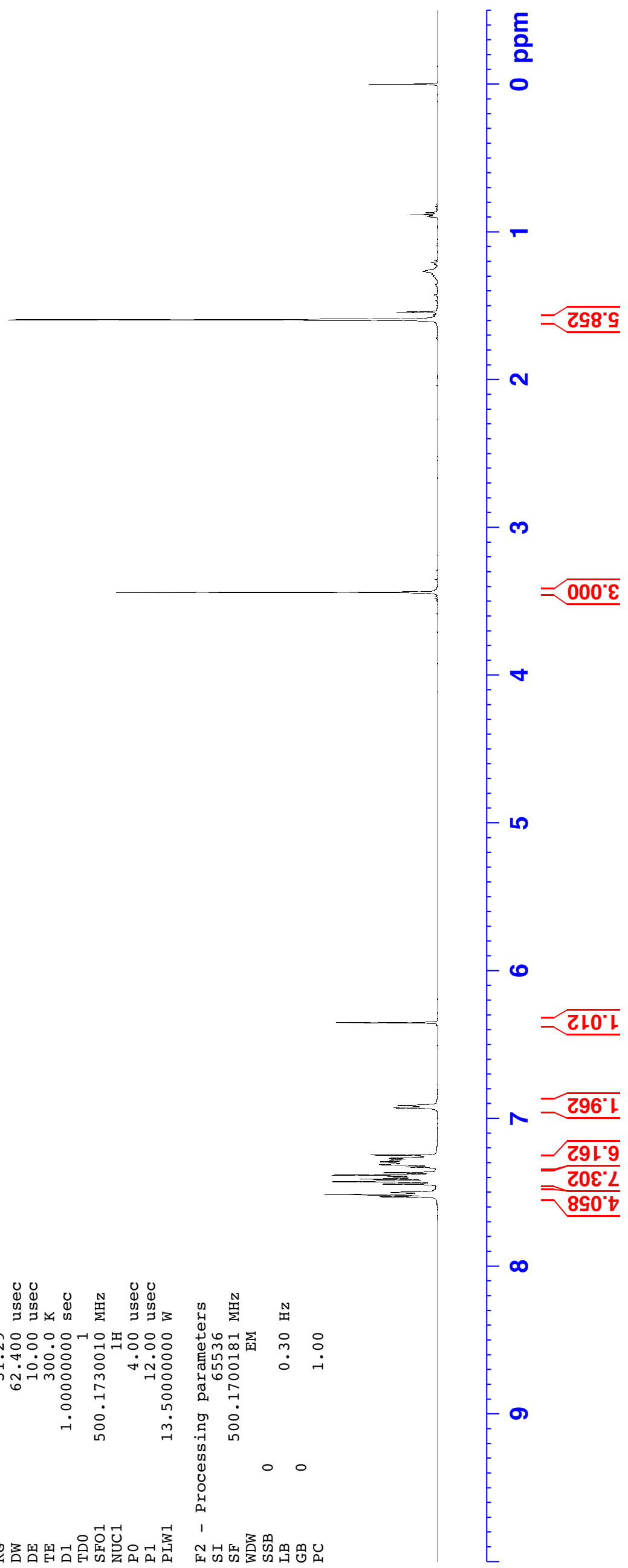

స్టి

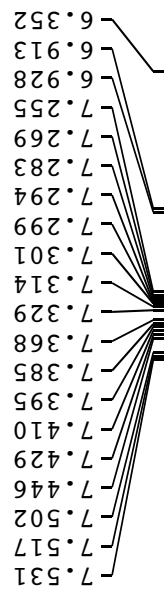

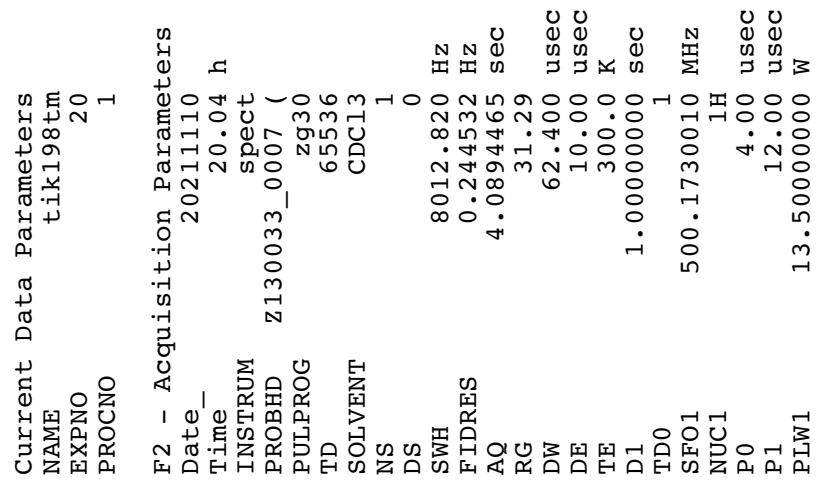

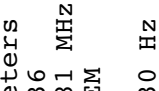

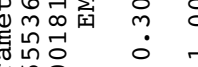

व 


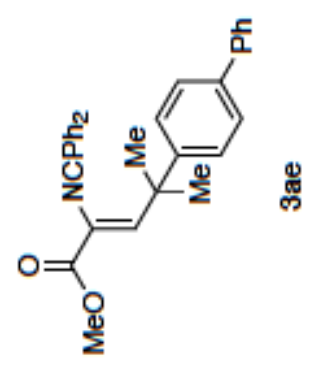

โ90*6Z

$\angle 69^{\circ} 6 \varepsilon$

IOL IS

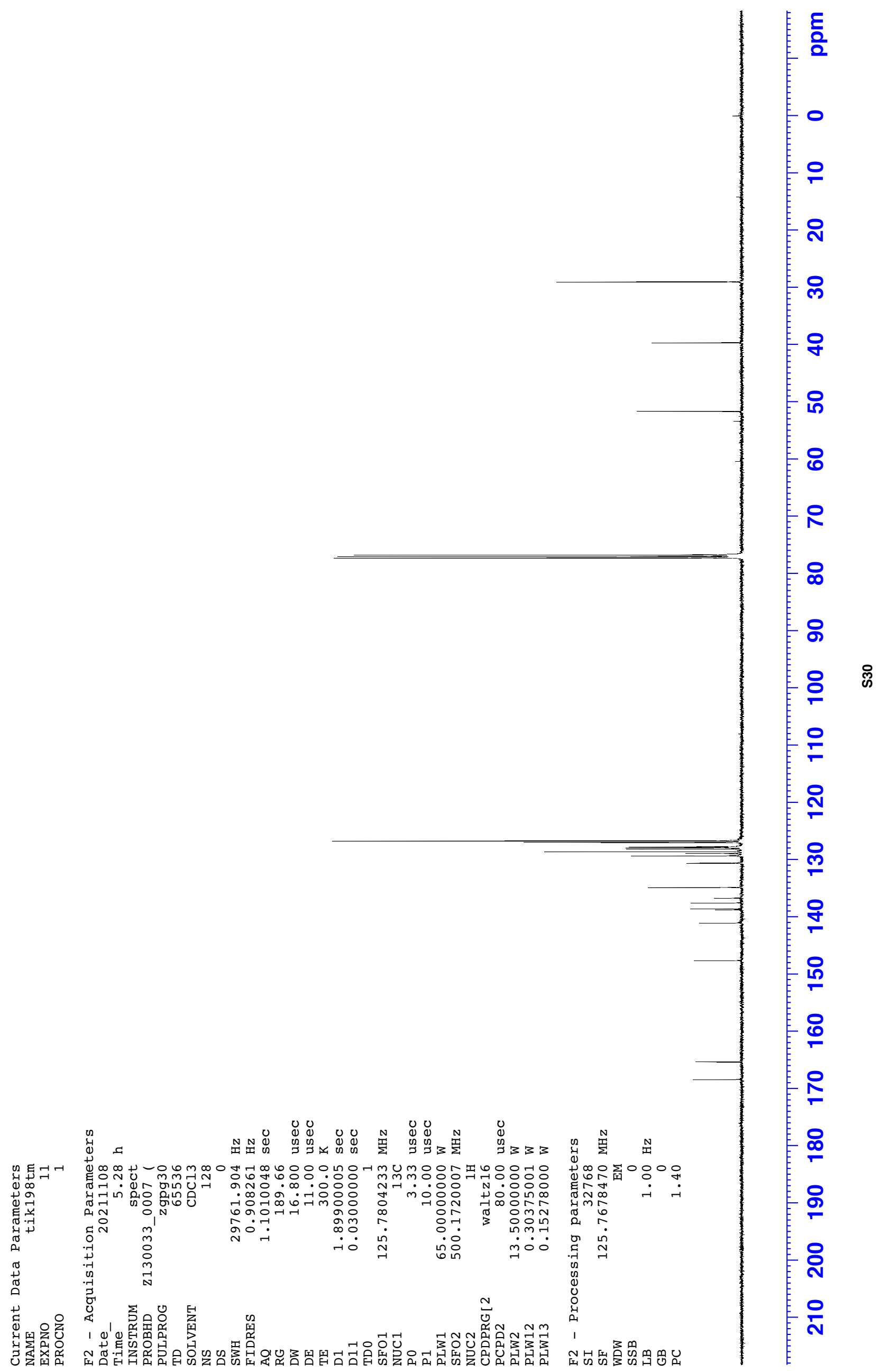

$9 S L \cdot 9 Z[]$

$766^{\circ} 9 Z \mathrm{I}$

$\angle T 0^{\circ} \angle Z T$

$\left.908^{\circ} \angle Z T\right]$

$60 \mathrm{r} \cdot 8 \mathrm{~T}$

$289^{\circ} 8 \mathrm{ZI}$

५ $96^{\circ} 8 Z \mathrm{I}$

$08 \varepsilon \cdot 6 乙 \mathrm{~L}$

I $9^{\circ} 0 \varepsilon \mathrm{I}$

$968^{\circ}$ ๑ โ I

$6 \succsim L \cdot 9 \varepsilon \mathrm{I}$

Z09 $\angle \varepsilon T$

$629 \cdot 8 \varepsilon[$

06L 8ह[ $]$

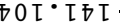

$989^{\circ} \angle \nabla[$

$98 \varepsilon \cdot \varsigma 9 \tau$

I6 $7^{\circ} 89 \mathrm{I}$ 


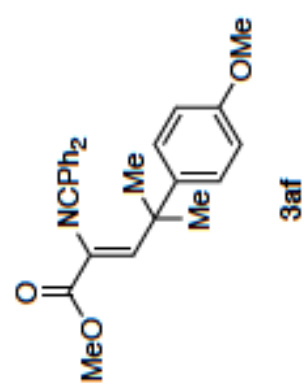

$6 \varepsilon \mathrm{G} \cdot \mathrm{T}$

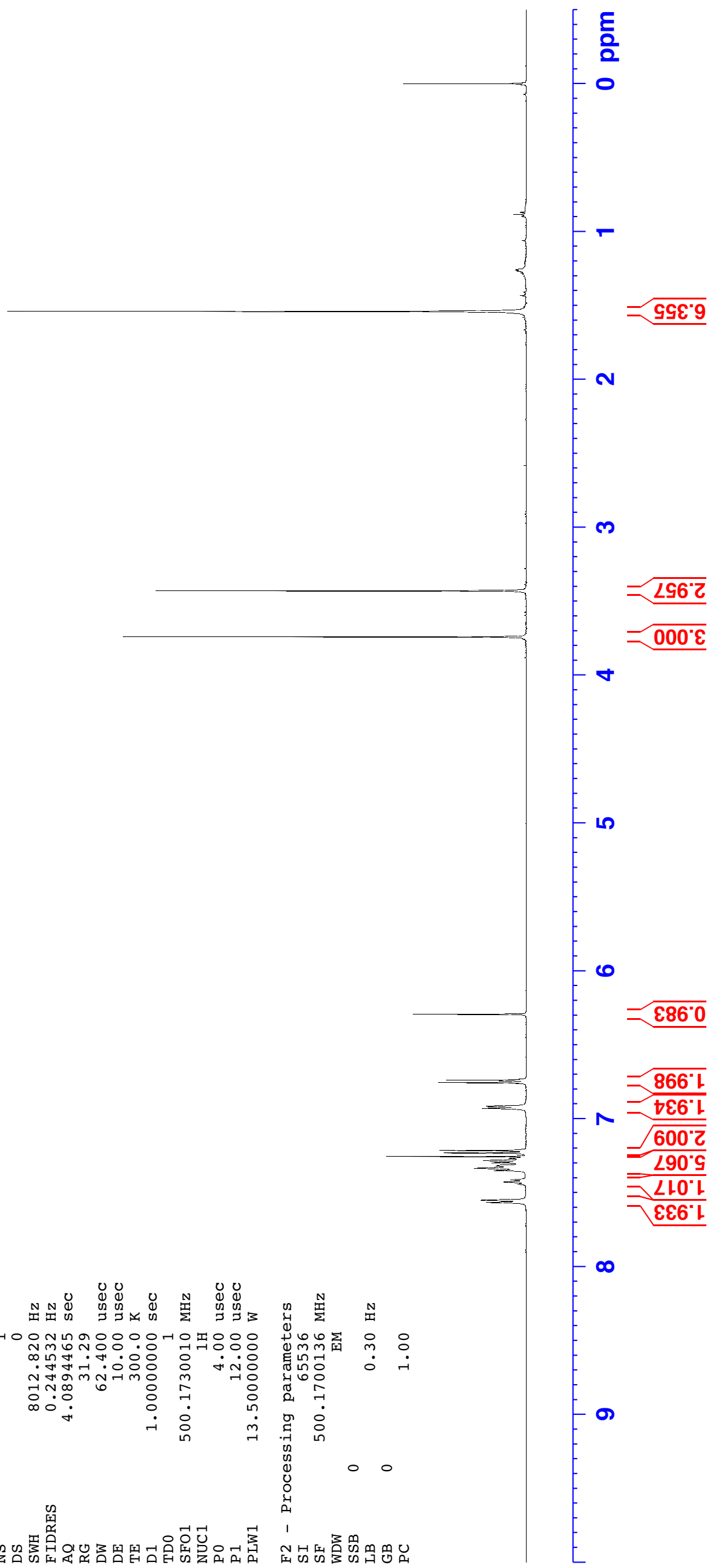

$\bar{\varnothing}$

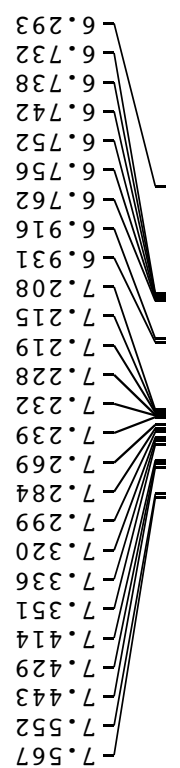

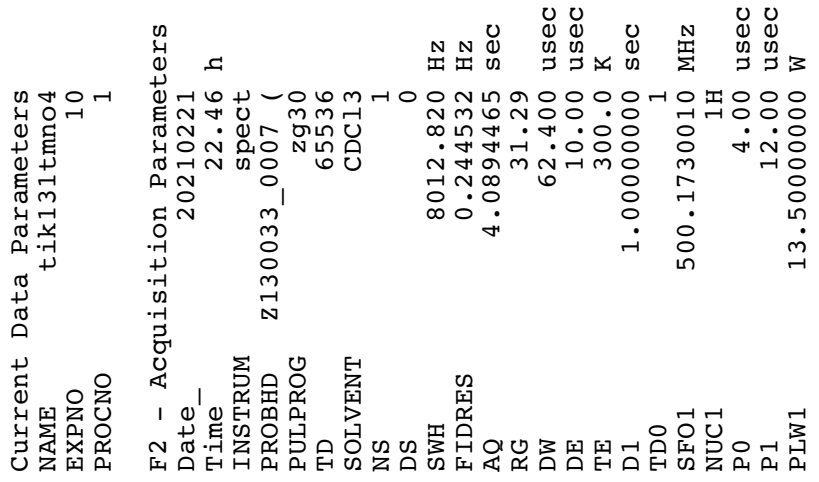




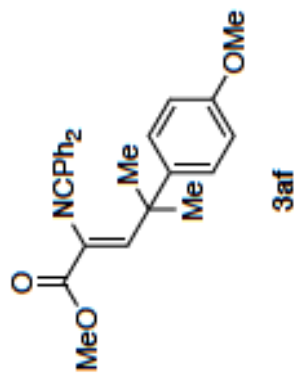

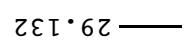

$80 \varepsilon \cdot 6 \varepsilon$

$0 \varsigma 9^{\circ}$ IS

$6 \varepsilon 乙 \cdot \varsigma \varsigma$

乙૬ع $\varepsilon \tau[$

[ $\angle Z \cdot \angle Z T]$

$Z 8 L \cdot \angle Z T$

$\varepsilon \varepsilon 6^{\circ} L Z T$

$\nabla \varepsilon 乙 \cdot 8 Z \tau$

$0 Z 6 \cdot 8 Z \mathrm{~T}$

$\angle L \varepsilon^{\circ} 6 Z \mathrm{I}$

IZ9 $0 \varepsilon \mathrm{I}$

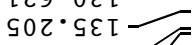

乙9L $9 \varepsilon \mathrm{I}$

$\mathrm{Z9Z} \angle \mathrm{L} \mathrm{L}$

$8 \varepsilon 8 \cdot 8 \varepsilon \mathrm{L}$

โ $88^{\circ} 0$ ธ โ

$009^{\circ} \angle G T$

$26 \varepsilon \cdot 99 I$

$6 \subseteq 7 \cdot 89 \mathrm{I}$

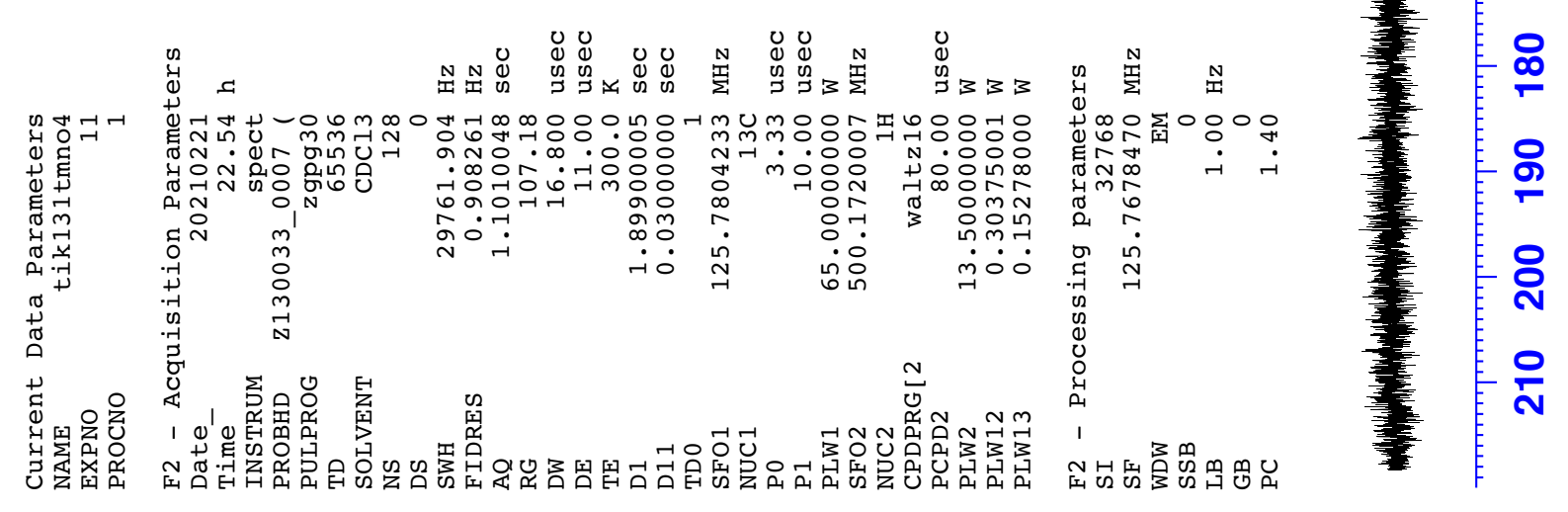




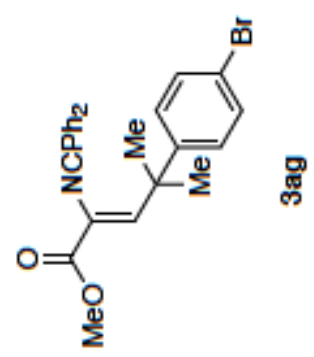

$\varepsilon I S^{\bullet} \tau$

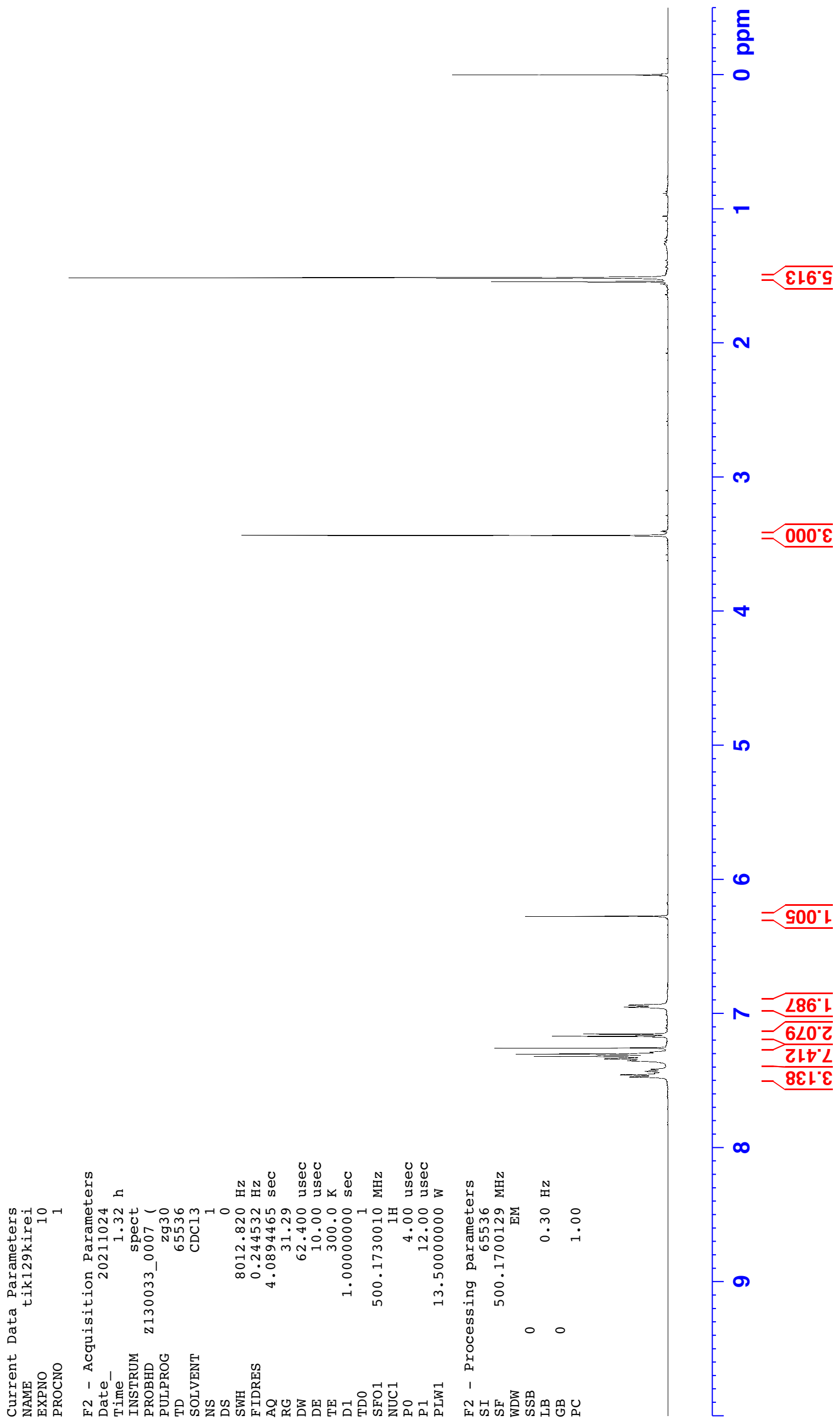

沛

$\mathrm{S} L Z \cdot 9$

$6 \varepsilon 6^{\circ} 9$

$\left.\varepsilon \subseteq 6^{\circ} 9\right]$

$\angle \nabla\left[{ }^{\circ} L\right]$

$L S[\cdot L]$

$\left.99 \mathrm{I}^{\circ} L\right]$

$9 \angle \mathrm{I}$.

$682^{\circ} L$

$\varepsilon 0 \varepsilon^{\circ} L$

โ乙 $\varepsilon^{\circ}$

$6 \varepsilon \varepsilon \varepsilon^{\circ} L$

$L I \nabla \cdot L]$

โE $\bullet\llcorner-$

$9 \nabla \pi \cdot L-$

$89 D^{\circ} L$

TLF

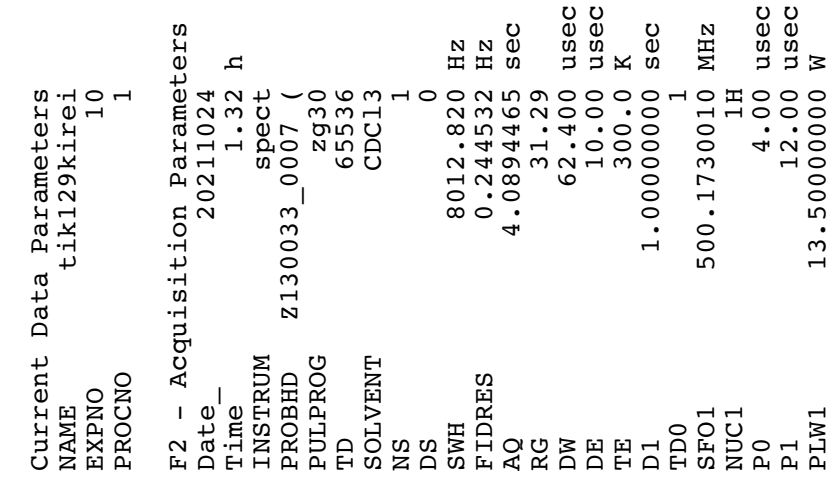

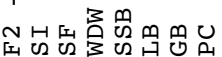




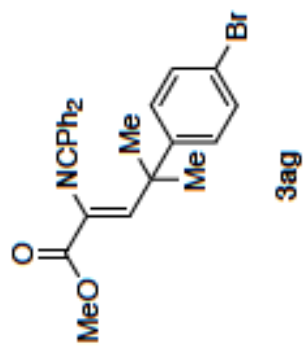

LIT $62 \longrightarrow$

$\angle โ 9 \cdot 6 \varepsilon-$

$9 Z L \cdot T S$

$\varepsilon \varepsilon S^{*} 6$ I I

$698^{\circ} \angle Z T$
$\left.Z 86^{\circ} \angle Z T\right]$

79

$9 \angle 0 \cdot 6 Z T$

$9 \angle \varepsilon \cdot 6 Z T$

$6 \succsim L \cdot 0 \varepsilon T \longrightarrow$

$000^{\circ}$ โ $\varepsilon \mathrm{L}$

$6 \angle 乙 \cdot \nabla \varepsilon \tau$

५ $99^{\circ} 9 \varepsilon \mathrm{L}$

[ $9 L^{\circ} L \varepsilon \mathrm{L}$

$\varepsilon\left[99^{\circ} 8 \varepsilon \tau\right.$

$8 \varepsilon 9^{\circ} \angle D T$ 


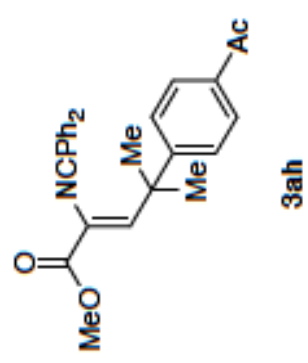

$\varepsilon L S^{\cdot} \mathrm{I}$

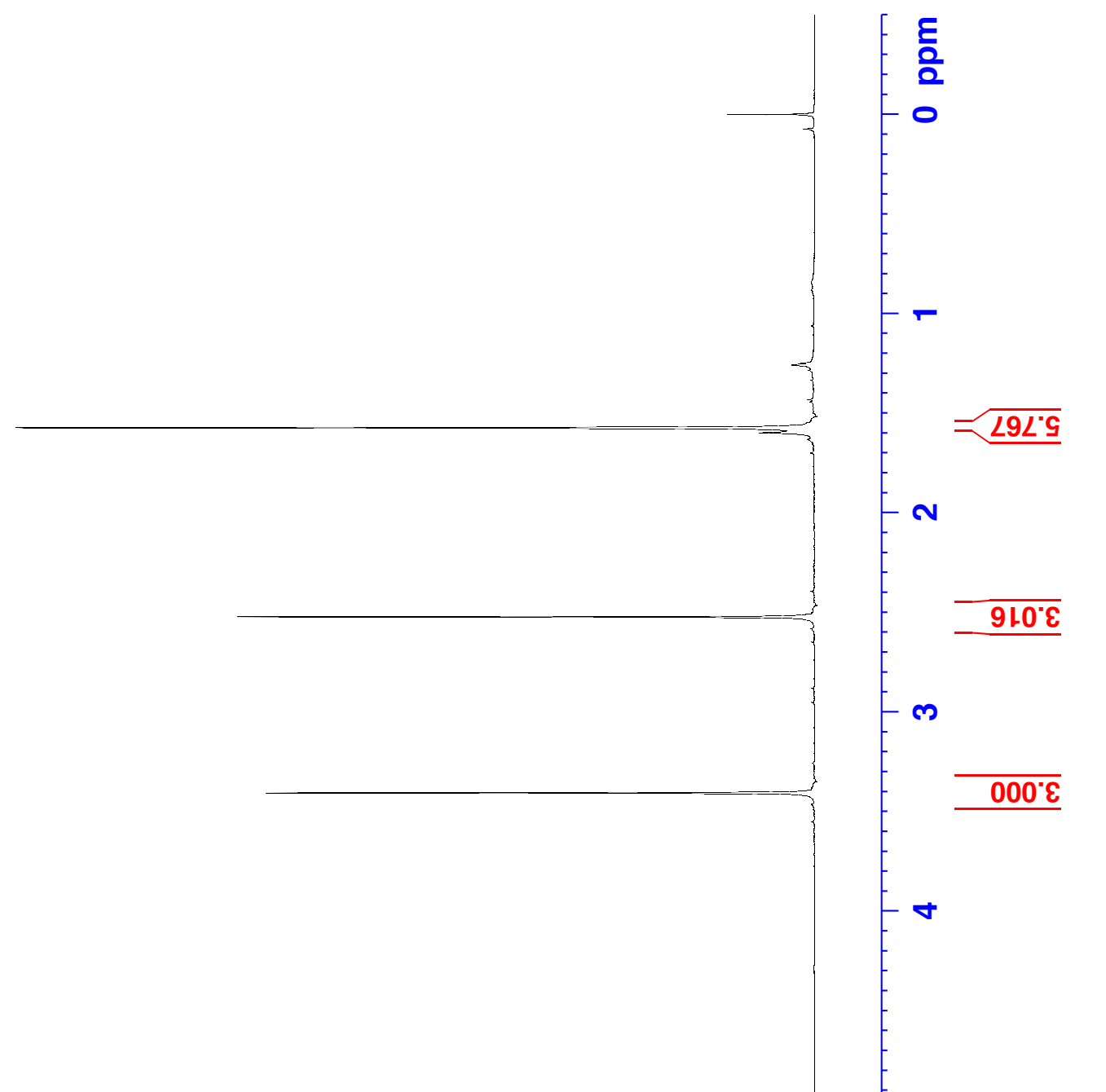

$\varepsilon 6 \tau^{*} \varsigma$

$6 \tau \varepsilon \cdot 9$

ऽะ6 9

$676 \cdot 9$

$\left.9 \angle \tau^{\circ} L\right]$

$90 \varepsilon \cdot L$

$97 \varepsilon^{\circ} L$

โ $\nabla \varepsilon \cdot L$

ऽऽ $\varepsilon^{\circ} L$

$06 \varepsilon^{\circ} L$

$\angle 0 \sigma^{\circ} L$

$6\left[\nabla^{\circ} L J\right.$

$6 L L \cdot L$

$96 L \cdot L$

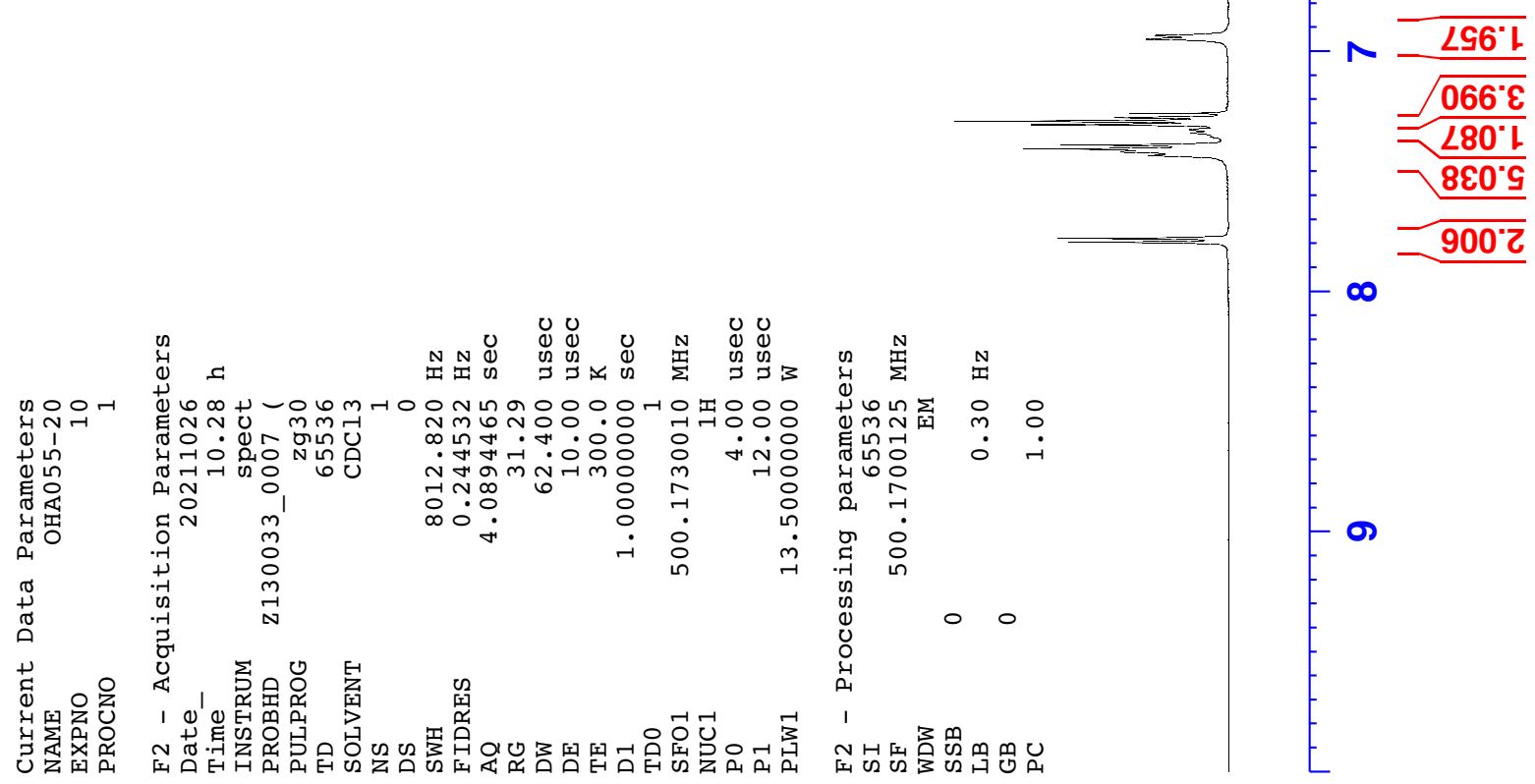

怘

L

$\bullet$ 


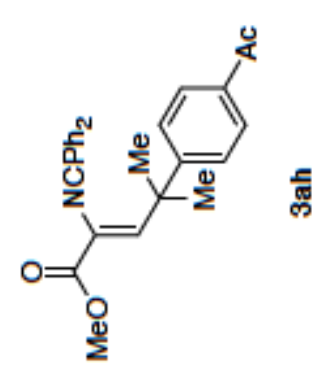

$0 \mp 5 \cdot 9 z-$
$\angle 00 \cdot 6 z=$

$6 \subseteq โ \cdot 0 \succsim$

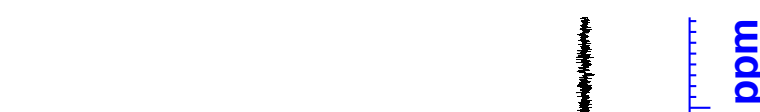

흘

$\varepsilon 69^{\circ}$ IS

$6 \varepsilon \mathrm{s} \cdot 9 Z[$

$\left.\begin{array}{l}\left.Z \angle L 8^{\circ} \angle Z T\right] \\ {[\angle Z[}\end{array}\right]$

$00 Z \cdot 8 Z T$

万I I $62 \mathrm{~T}$

$\angle 9 \varepsilon \cdot 6 Z I$

$9 L L \cdot 0 \varepsilon \mathrm{L}$

6Е๐

ธ०L $\nabla \varepsilon L$

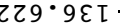

$\angle S 6^{\circ} \angle E \mathrm{~L}$

$6 \varepsilon \mathrm{\circ} \cdot 8 \varepsilon \mathrm{I}$

$โ L Z \cdot \nabla \subseteq \tau$

$\angle 6 I \cdot 59 \mathrm{I}$

Ћ๘ $\cdot 89 \mathrm{I}$

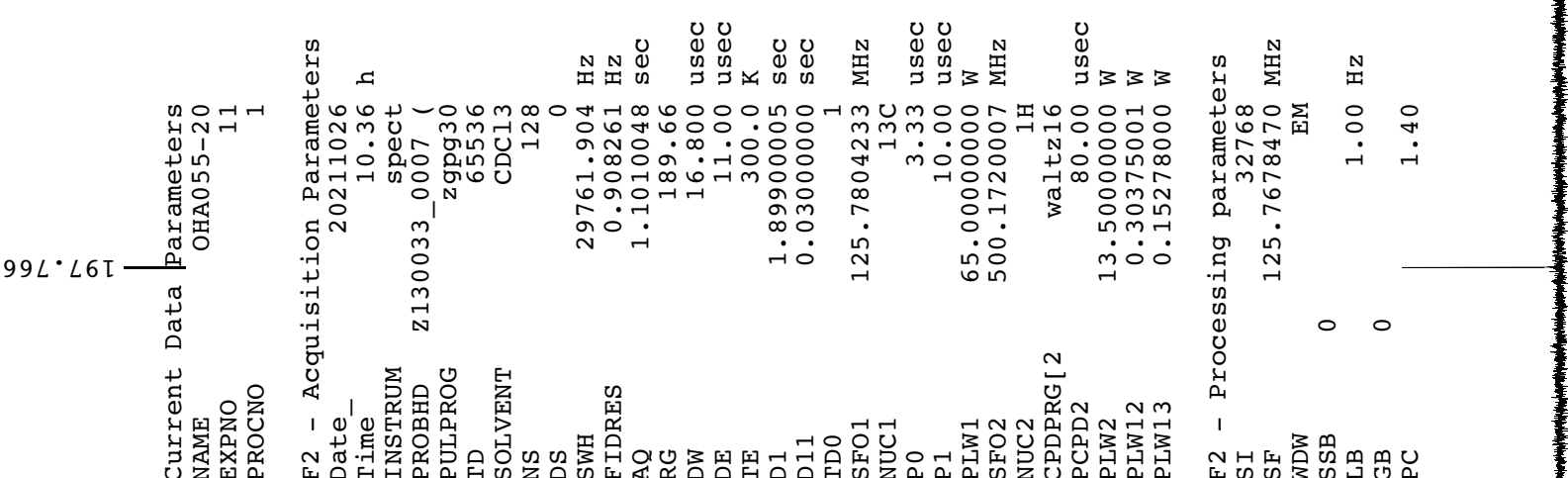



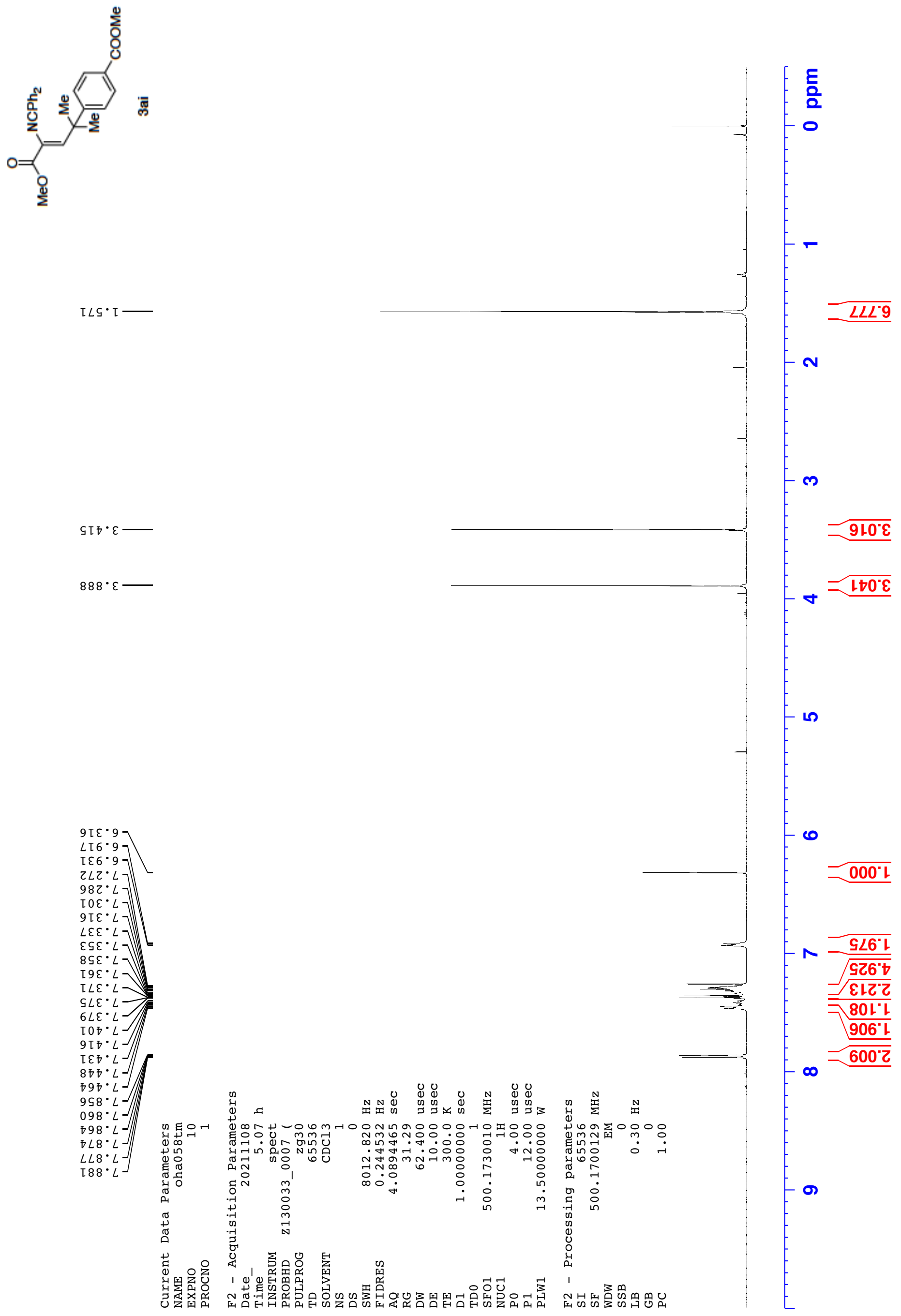

$\widehat{\mathscr{D}}$

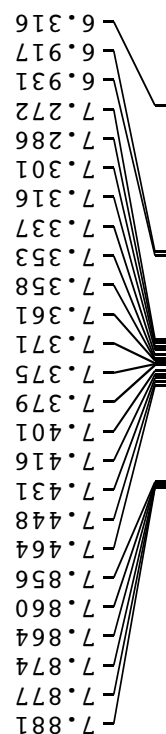

量工

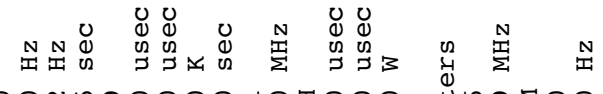

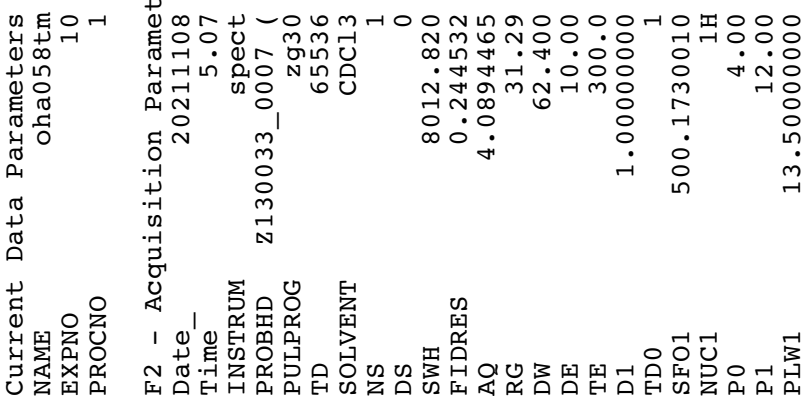

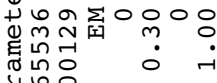

$[88 \cdot L$ 


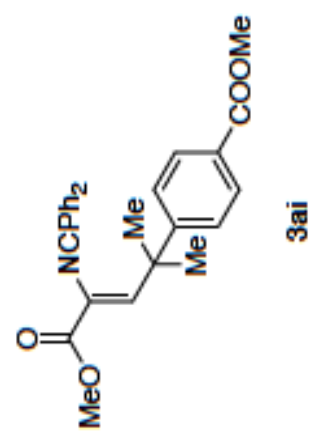

$000 \cdot 62$

$\varepsilon \varpi \mathrm{I} \cdot 0 \emptyset \square$

$069^{\circ}$ IS $\longrightarrow$

$926^{\circ}$ [S $\longrightarrow$

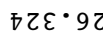

$\left.8 \varepsilon G^{\circ} L Z T\right]$

$898^{\circ} \angle Z T$

$926^{\circ} \angle Z T$

SLO.6ZI

$99 \varepsilon^{\circ} 6 Z T$

L6E・6ZT

Ђ $L \cdot 0 \varepsilon \mathrm{L}$

$08 \varepsilon \cdot \nabla \varepsilon \tau$

$099^{\circ} 9 \varepsilon \mathrm{L}$

$0 \angle 6^{\circ} L \varepsilon[$

$6 \varepsilon \mathcal{G}^{\circ} 8 \varepsilon[$

$800^{\circ}$ 万รI

$0 \varepsilon 乙 \cdot \varsigma 9 \mathrm{~L} \longrightarrow$

$\mathrm{I} 80^{\circ} \angle 9 \mathrm{I}$

$\varepsilon \angle D^{\circ} 89 \mathrm{I}$

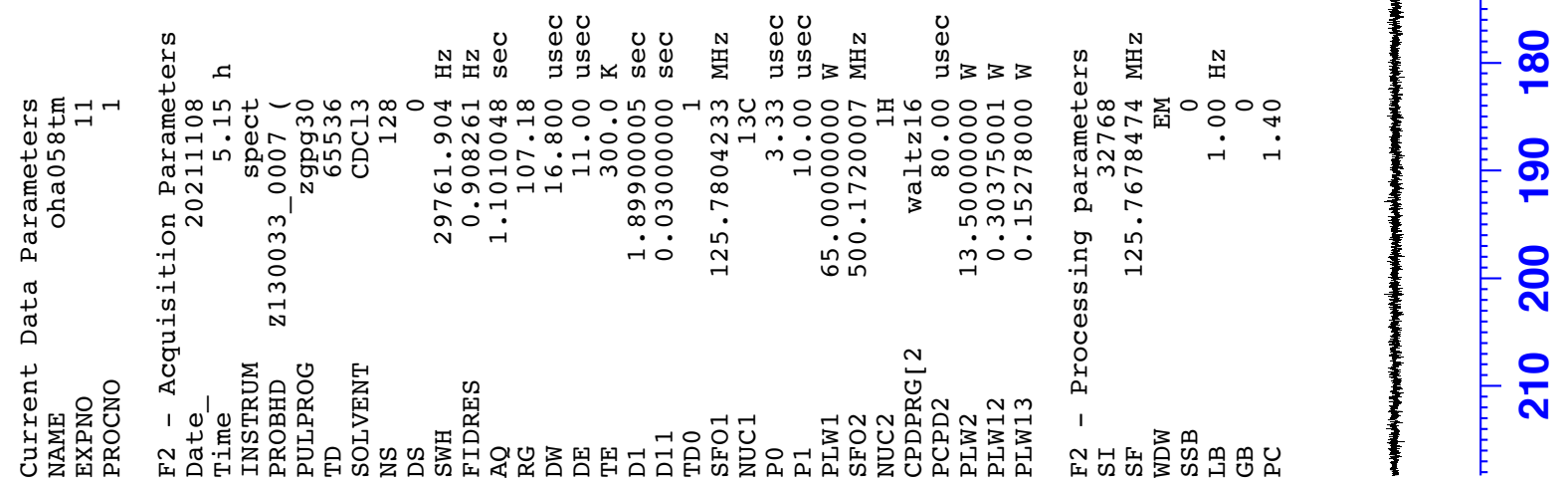




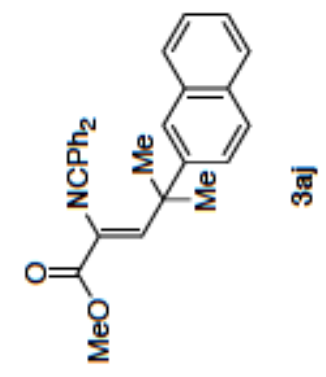

$\varepsilon \varsigma 9^{\circ} \mathrm{L}$

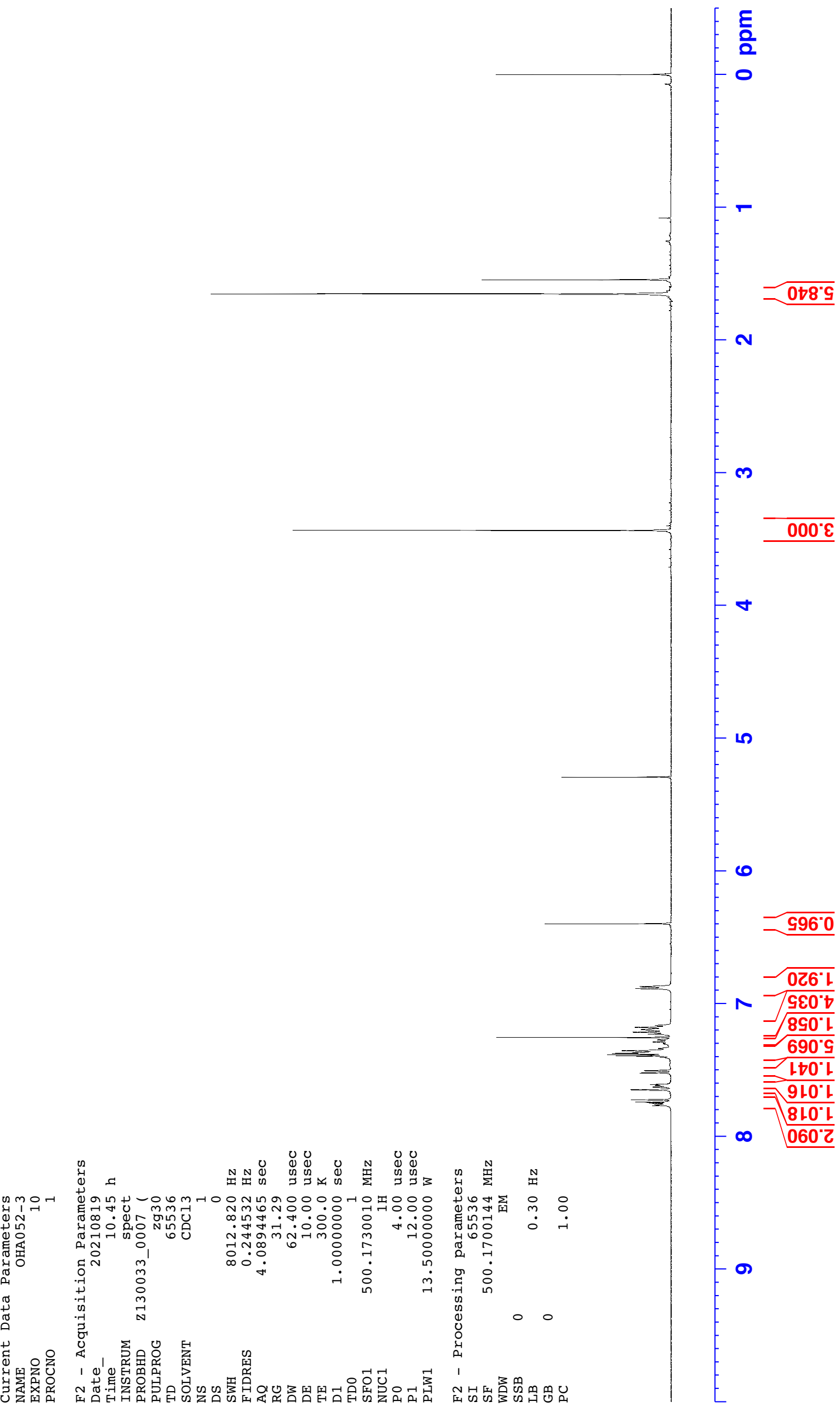

禺

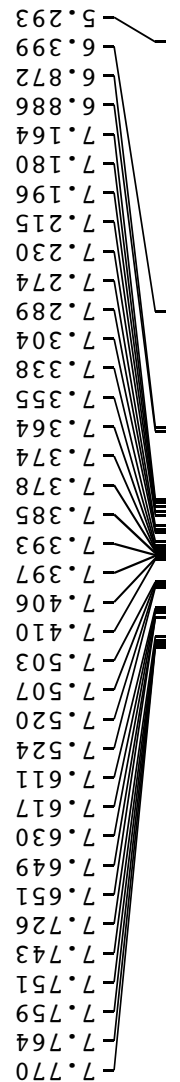

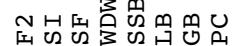




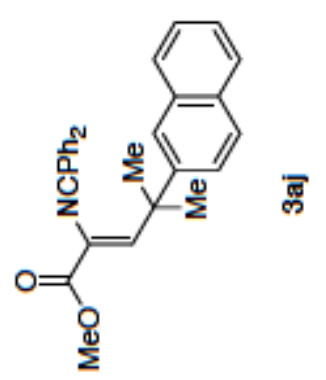

$820 \cdot 62$

โ๑0・0ゥ—

$699^{\circ}$ IS

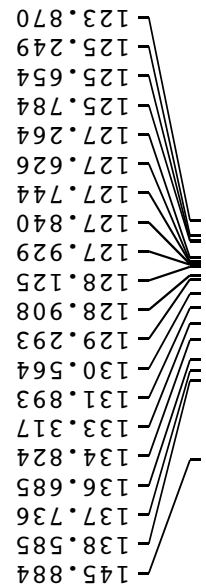

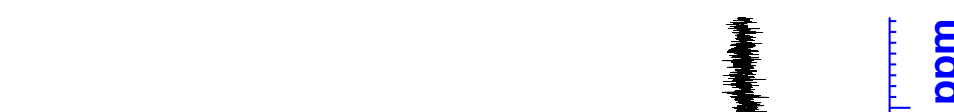

등

으

오

요

우

우

8

오

$\infty$

৪

8 \&

으

กิ

옴

온

농

웅

ธLE. $99 \mathrm{~L}$

$\varepsilon 6 \varepsilon^{\circ} 89 \mathrm{I}$

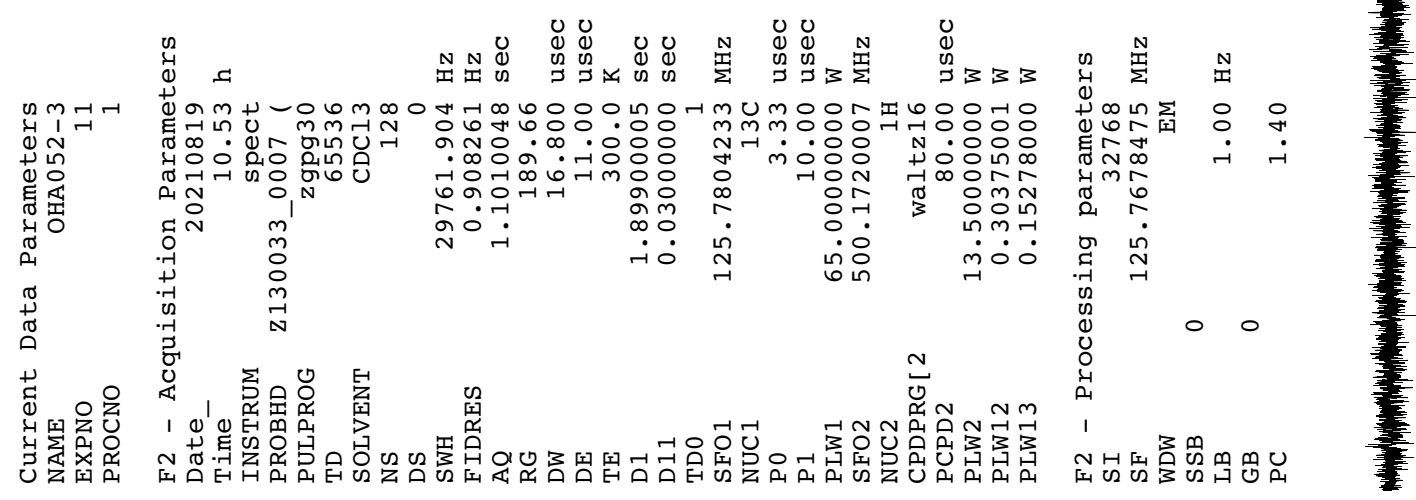

온

$\stackrel{\circ}{-}$

옹

ㅇํ

은 

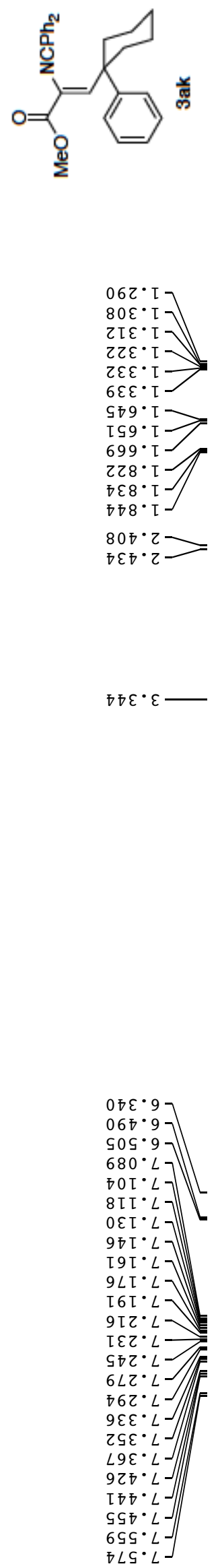

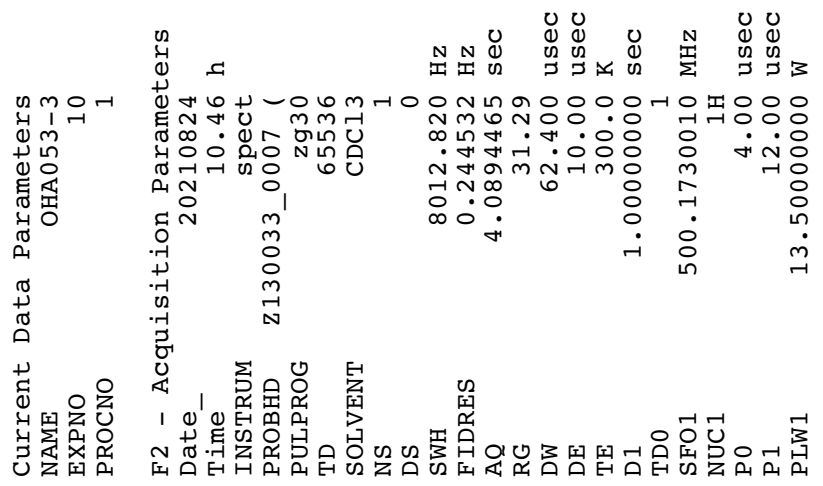

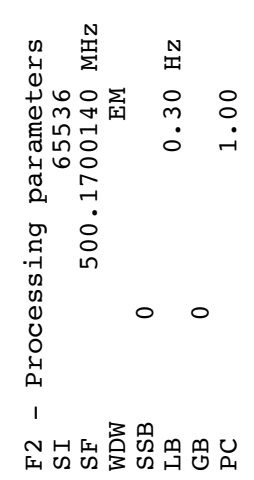

F
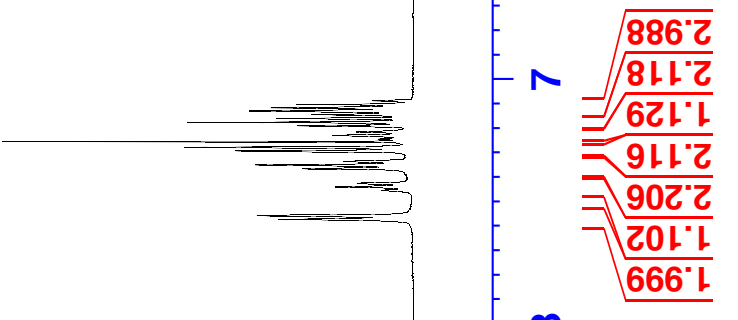


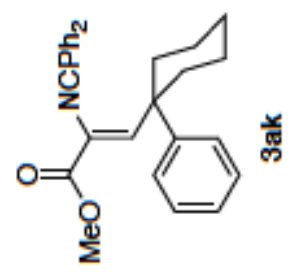

$\varsigma \varepsilon 乙 \cdot \varepsilon 乙-$

$090 \cdot 9 z$

$\tau \varepsilon \tau \cdot 9 \varepsilon-$

$\varsigma 0 乙 \cdot \varepsilon \succsim$

ธ9ซ・โร

ง8. 92

$80 L \cdot 9 Z T$

$\varepsilon \subseteq 6^{\circ} \angle Z \mathrm{~L}$

$\varepsilon 86^{\circ} \mathrm{LZT}$

I L $L \cdot 8 Z$ T

0โع 6 С

$69 \mathrm{~s} \cdot 0 \varepsilon \mathrm{T}$

$\varepsilon \subseteq \varsigma^{\bullet} \nabla \varepsilon \mathrm{L}$

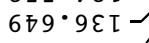

$\mathrm{S} 8 L^{\circ} L \varepsilon \mathrm{L}$

$9 \varepsilon 8 \cdot 8 \varepsilon \tau$

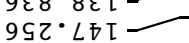

$9 \angle 0 \cdot 59 \mathrm{~L}$

$\varepsilon I 7 \cdot 89 \mathrm{I}$

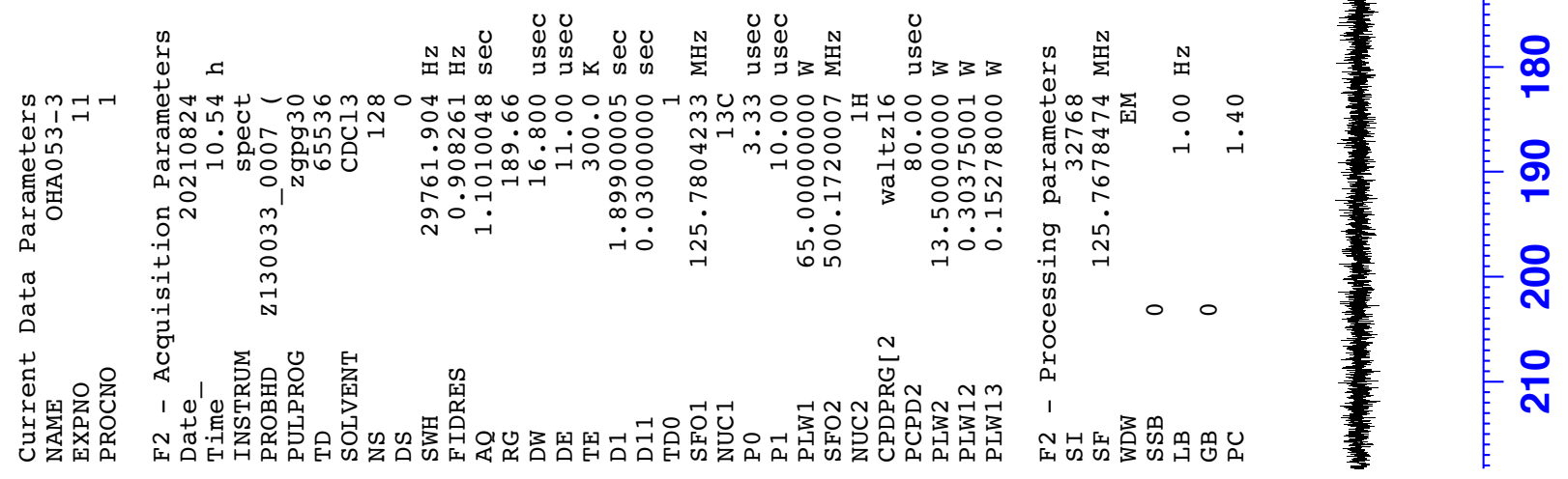




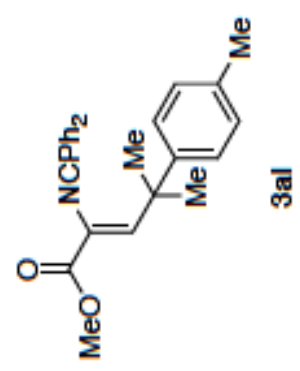

$9 \varepsilon S^{*} \mathrm{I}$

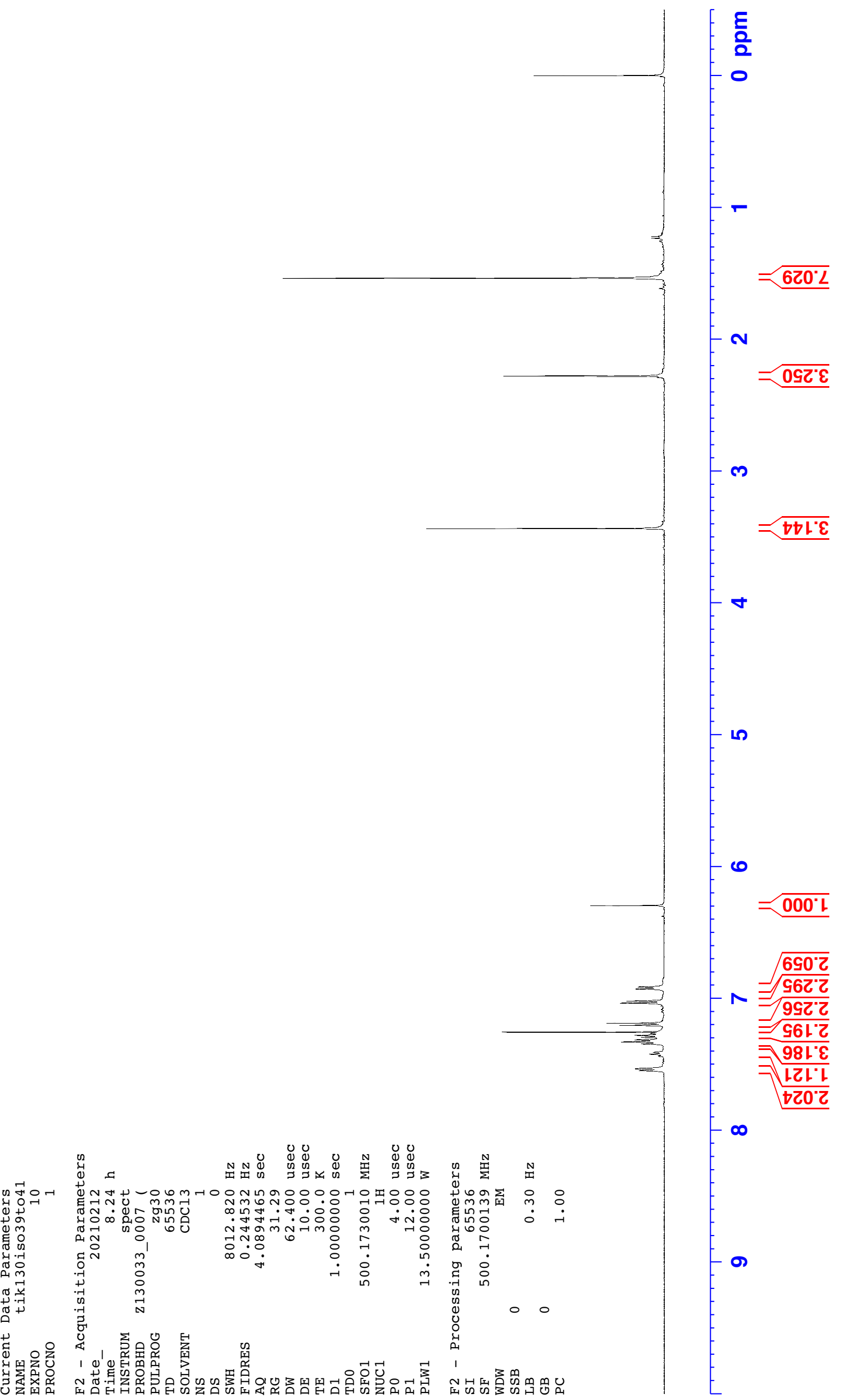

$9 \varepsilon \varpi \cdot \varepsilon$

$\stackrel{9}{6}$ 


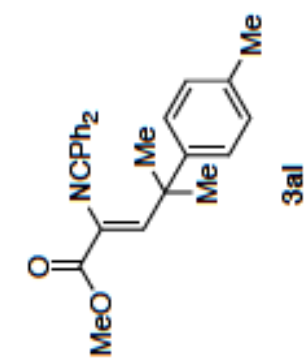

$8 \angle 8^{\circ} 0 Z$

$0 \varepsilon 0 \cdot 6 Z$

$0 \mp 9 \cdot 6 \varepsilon$

9 9 $9^{*}$ I

$\angle \varepsilon[\cdot 9 Z[]$

$\varepsilon\left[6^{\circ} L Z T\right.$

ఐぇて・8ZI

ЂてL・8ZI

$\angle 68^{\circ} 8 Z \mathrm{~T}$

$99 \varepsilon \cdot 6 Z \tau$

$86 \mathrm{~S}^{\circ} 0 \varepsilon \mathrm{L}$

$890^{\circ}$ ฯ โ

$96 \mathrm{I} \cdot \mathrm{\varsigma} \mathrm{L}$

乙LL $9 \varepsilon \tau$

५ $8 \mathrm{C}^{\circ}\llcorner\varepsilon[\rfloor$

与 $88^{\circ} 8 \varepsilon \mathrm{L}$

$0 \nabla 9^{\circ} \mathrm{StL}$ 


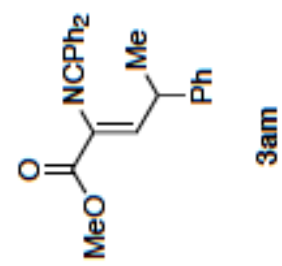

$8 \pi 2 \cdot \tau \longrightarrow$

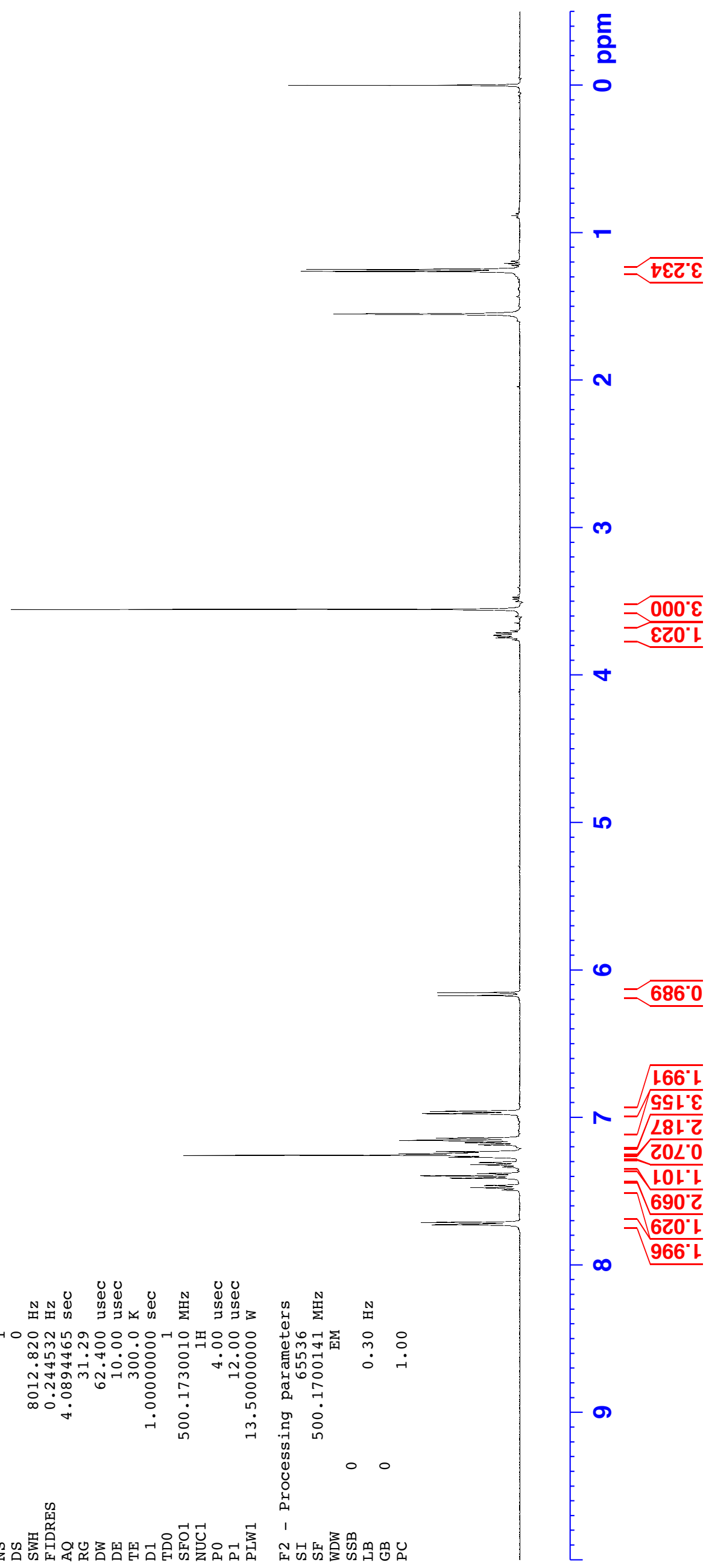

$\stackrel{8}{\infty}$

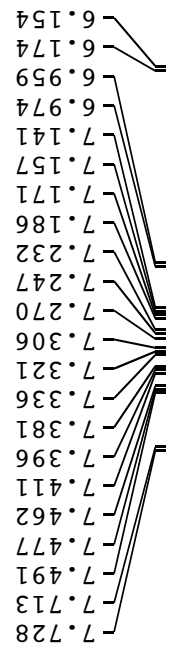

$n$
na

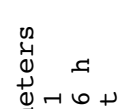

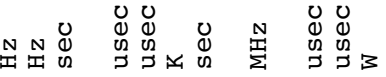

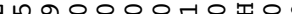

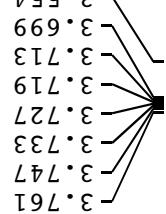

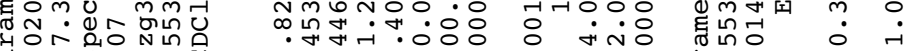

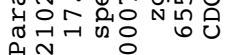

घू ले

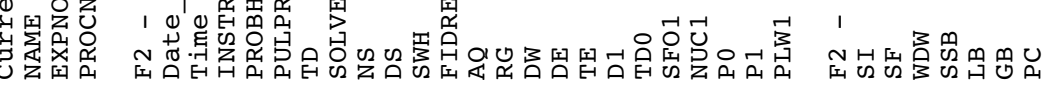




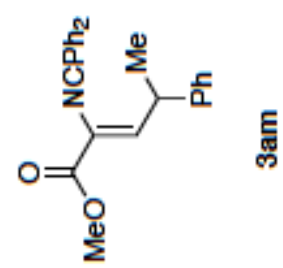

$008 \cdot 02$

$00 \varepsilon^{\circ} \angle \varepsilon$

$\varepsilon 08^{\circ} \mathrm{TS}$

$0 \subseteq z \cdot 9 z$

$Z \varepsilon Z \cdot L Z \mathrm{I}$

$8 \pi \mathrm{I} \cdot 8 \mathrm{I}$

$\angle 8 \mathrm{I} \cdot 8 \mathrm{LT}$

$68 \mathrm{D} \cdot 8 Z \mathrm{I}$

$\angle 90^{\circ} 62 \mathrm{~T}$

ع97.6ZI

9โZ.0हโ

9โ6 $0 \varepsilon[$

ธع9 $9 \varepsilon \mathrm{L}$

SS下 $8 \varepsilon \mathrm{L}$

0ะ6.8ह [

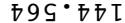

ธ98・ซ9โ —

$59 L \cdot 0 L I$

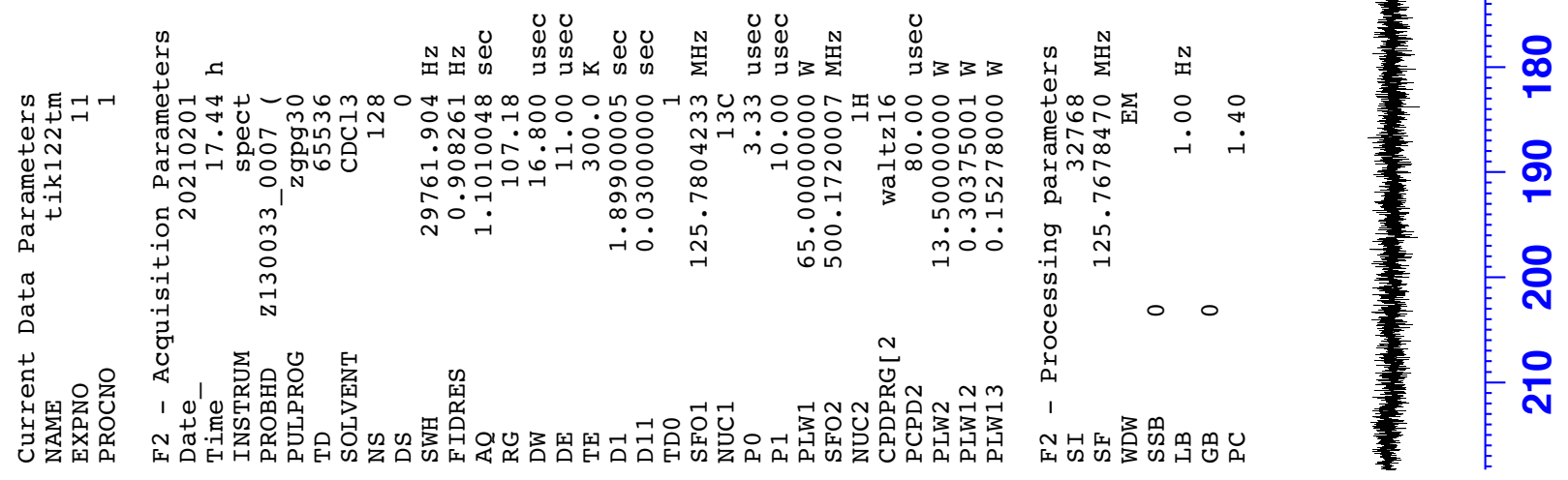



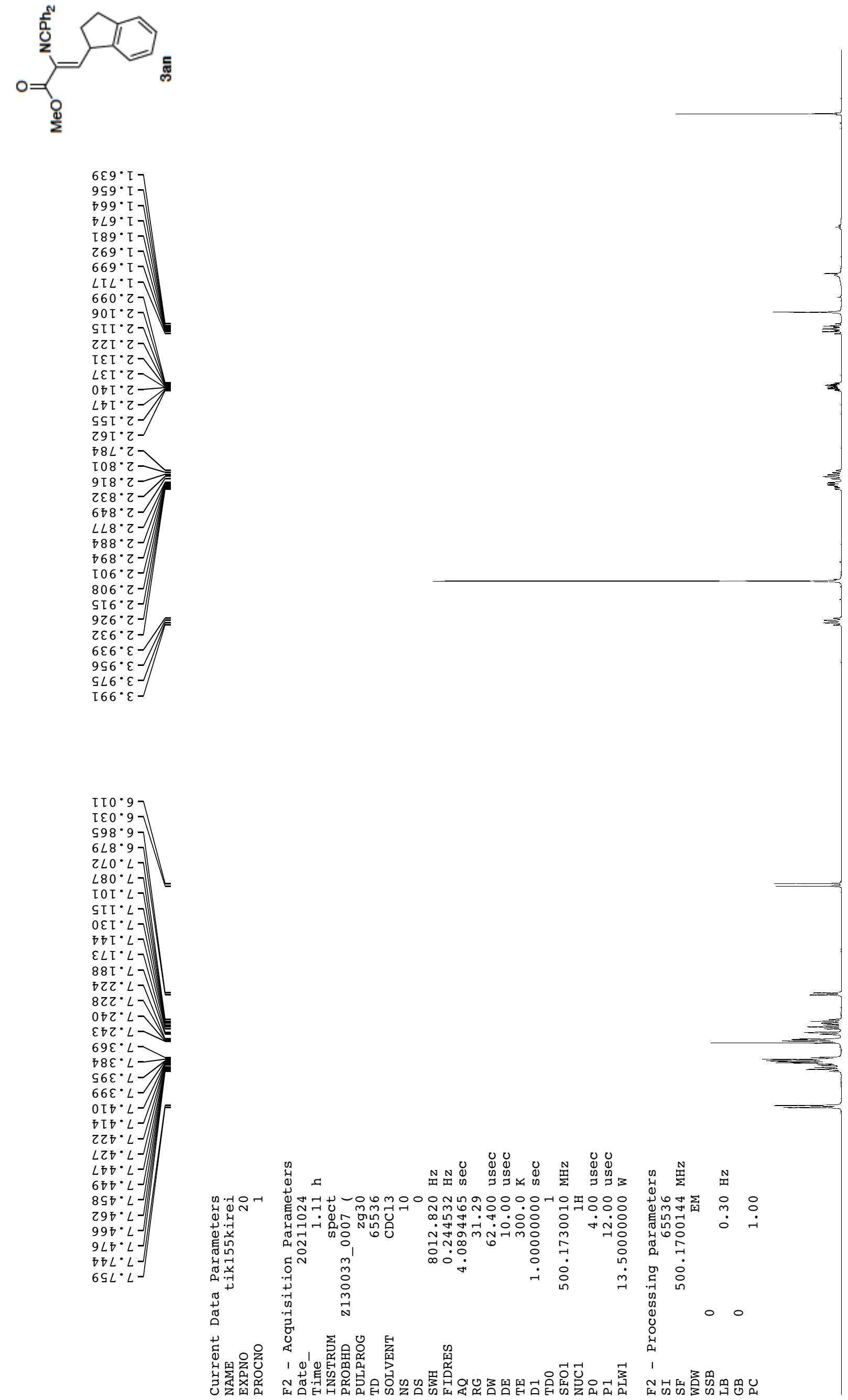

- N

$186^{\circ} 0$

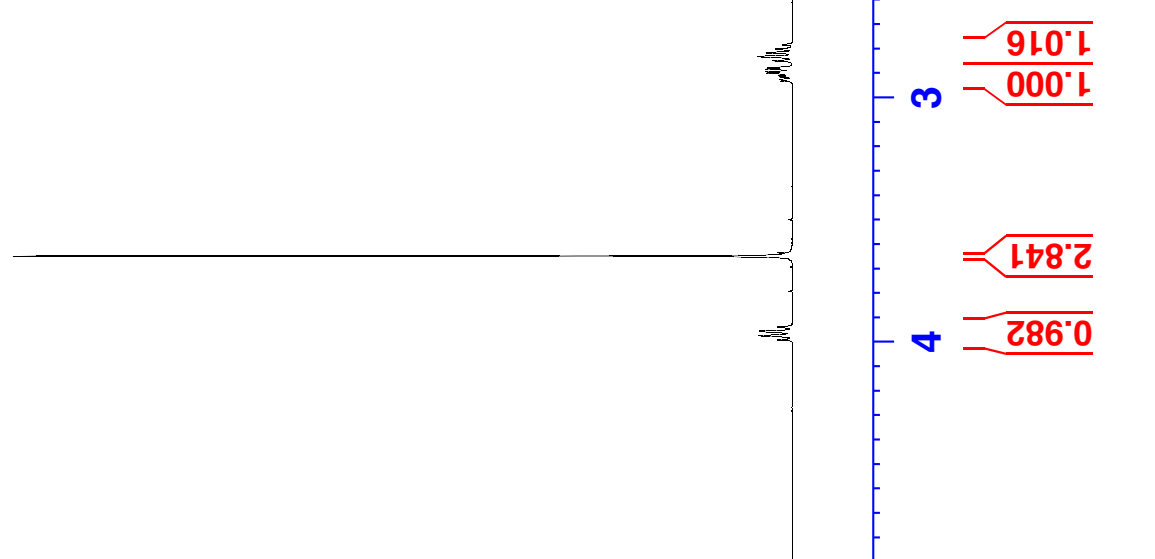

F

( )

$6 \longdiv { 9 9 6 . 0 }$

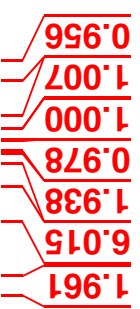

$\infty$ 


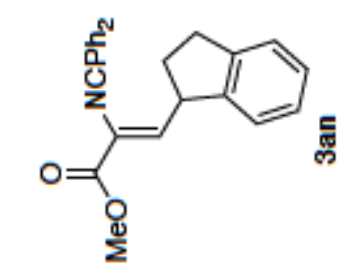

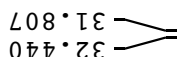

$69 乙 \cdot \varepsilon \varpi$

$06^{*}$ โ

โ乙E・ゥ乙โ

$8 \angle Z \cdot 9 Z I$

SOL・9ZI

E9S $\angle Z$

$\angle S T \cdot 8 Z T$

万I 8 •

6น

ZE与 6 СТ

$8 \angle 6^{\circ} 0 \varepsilon \mathrm{L}$

५ $9^{\circ} 9 \varepsilon \mathrm{L}$

प८० $8 \varepsilon \mathrm{L}-$

$90^{\circ} 07 \mathrm{~T}$

$00 L^{\circ} \varepsilon \bar{L}$

५ธโ ऽฐโ

$666^{\circ}$ ๑9

$\nabla 66^{\circ} 0 L \mathrm{~L}$

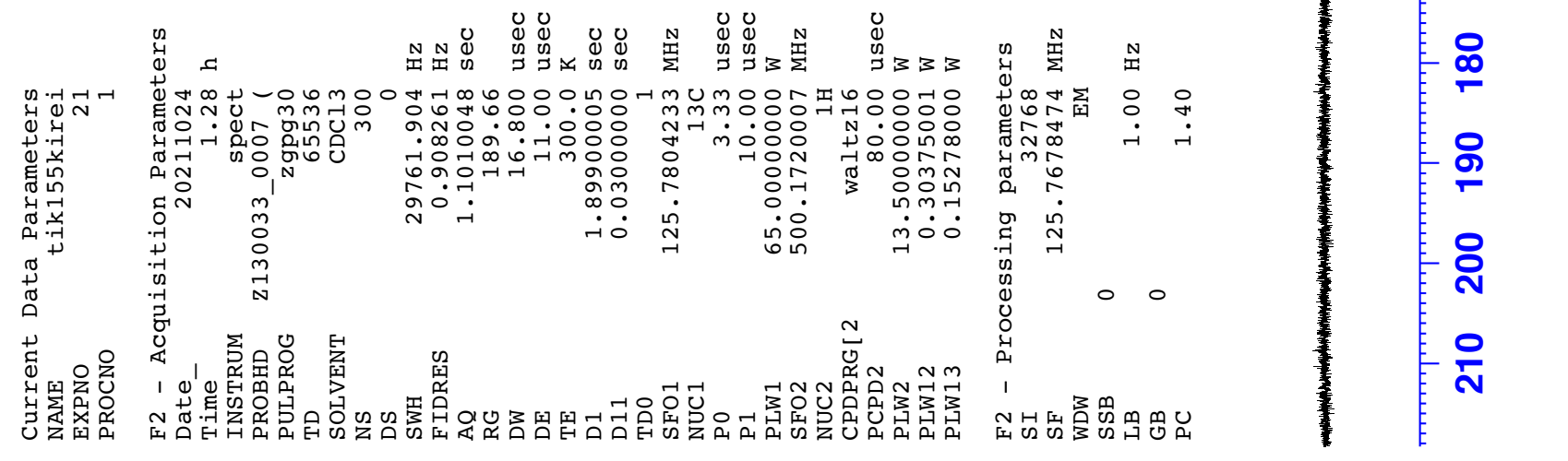



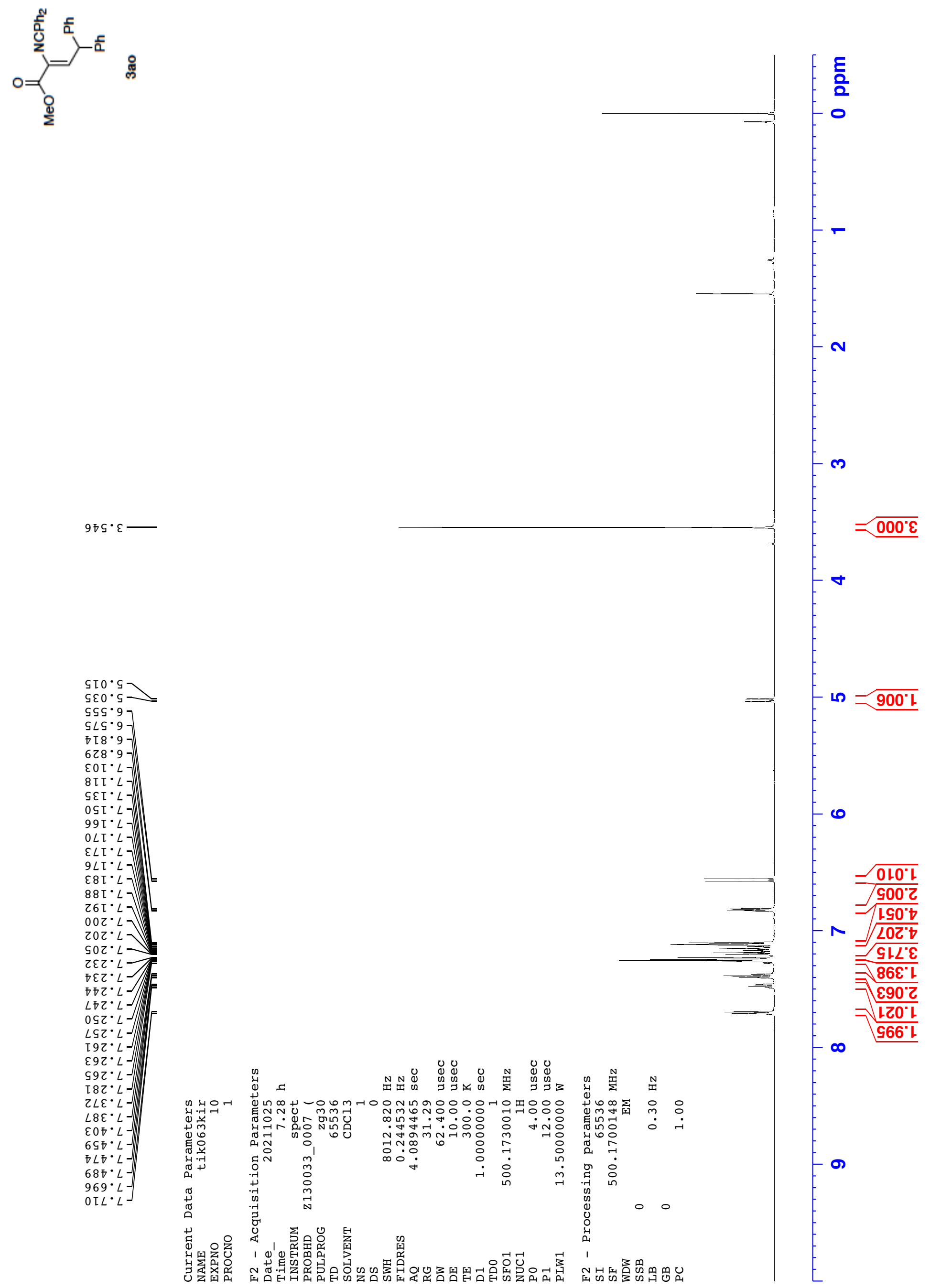

导

SE0.S

ऽ૬ $\cdot 9$

$S \angle S \cdot 9]$

$628 \cdot 9$

$\left.\varepsilon 0 I^{\circ} L\right]$

$\varsigma \varepsilon \tau \cdot L]$

$0 S[\cdot L]$

$99 \tau \cdot L]$

$\varepsilon L I \cdot L]$

$9 L I \cdot L]$

$\left.88 I^{\circ} L\right]$

$26 I^{\circ} L$

$00 Z^{\circ} L$

与०乙 $L$

乙ह ${ }^{\circ} L$

$\nabla \varepsilon 乙 \cdot L$

$\nabla \nabla 2 \cdot L J$

$0 \subseteq 乙 \cdot L J$

$\angle S Z \cdot L J$

โ $92^{\circ} L$

$\varepsilon 9 z^{\circ} L-$

$\left.99 Z^{\circ} L\right]$

$Z \angle \varepsilon^{\circ} L$

$L 8 \varepsilon \cdot L$

$\varepsilon 0 \sigma^{\circ} L$

$6 S \sigma^{\circ} L$

$\nabla \angle D^{\circ} \angle$

$680^{\circ} L$

$969^{\circ} L-$

$0[L \cdot L]$

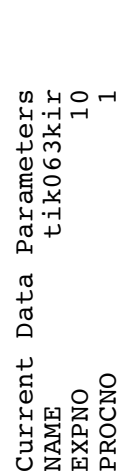

苟

先 要 N

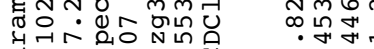

菏

सत्

व.

$\begin{array}{cc}0 & 0 \\ -7 & 0 \\ -7 & 0 \\ -1 & 0 \\ 0 & 0 \\ 0 & 0\end{array}$

获 
O=

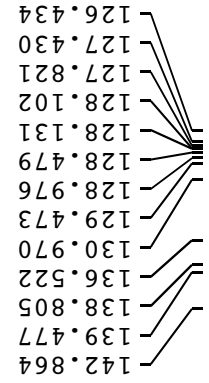

$809^{\circ}$ ธ9

$6 L \tau \cdot \tau L I$

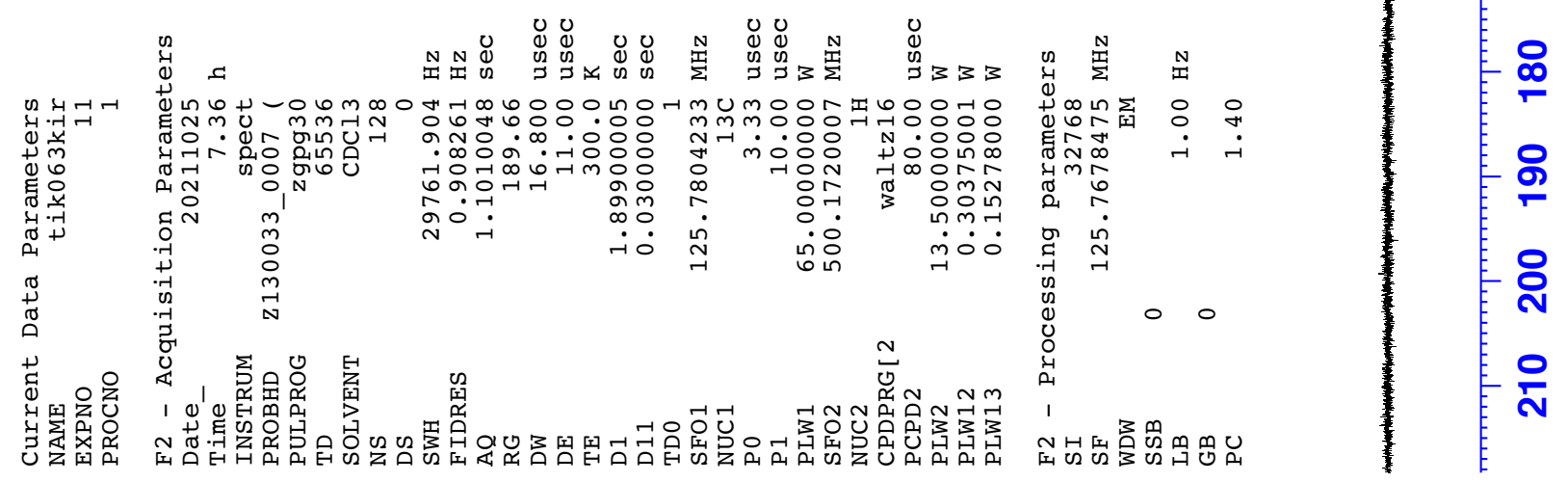



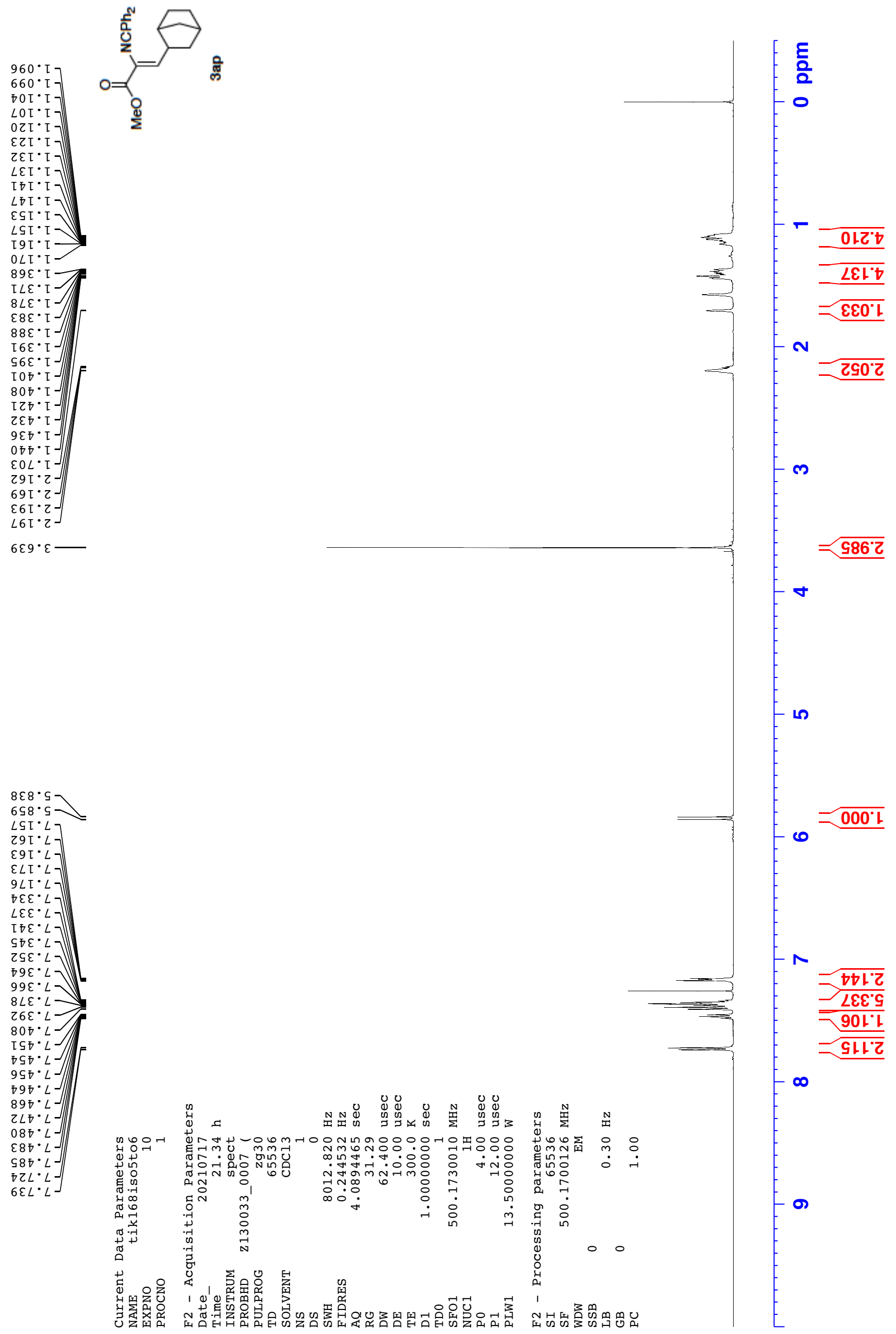

Бึ
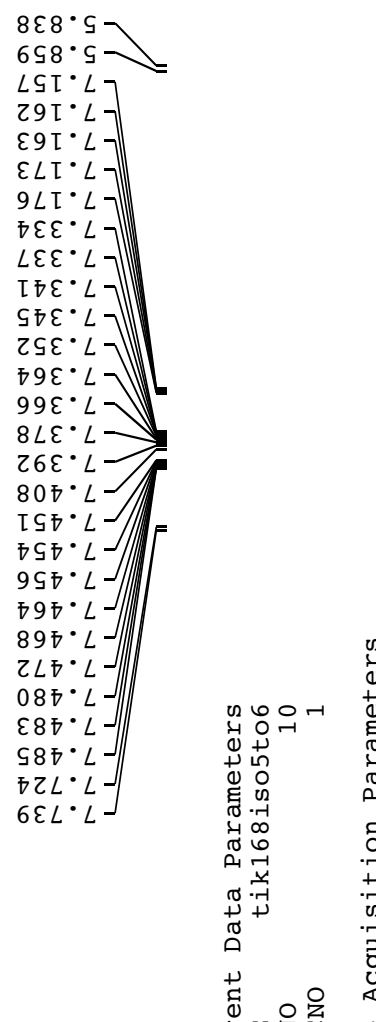

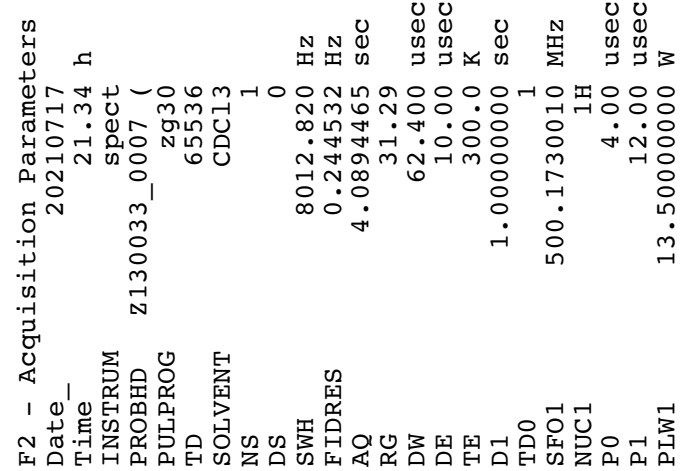




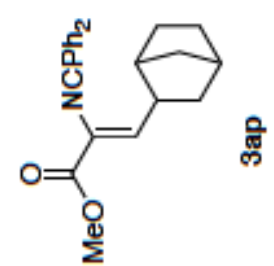

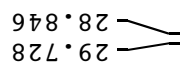

$\varepsilon 66 \cdot 9 \varepsilon$

$9 Z \nabla \cdot 9 \varepsilon$

$2 \subseteq 9^{\circ} \angle \varepsilon$

$86 L \cdot 6 \varepsilon$

SI6.

ธ98 ${ }^{\circ}$ โ

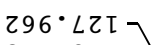

$\varepsilon \nabla \tau \cdot 8 Z T$

与 $8 \mathrm{C}^{\circ} 8 \textrm{ZT}$

$690^{\circ} 6 Z \mathrm{I}$

ZLF $62 \mathrm{~L}$

$\varepsilon \nabla 8^{\circ} 0 \varepsilon \mathrm{I}$

$\varepsilon \angle 9 \cdot 9 \varepsilon$

$\mathrm{S} \mathrm{T} \cdot L \varepsilon \mathrm{T}$

$00.6 \varepsilon \mathrm{I}$

L乙E. $९ 9 \tau$

$Z \angle 9^{\circ} O L I$

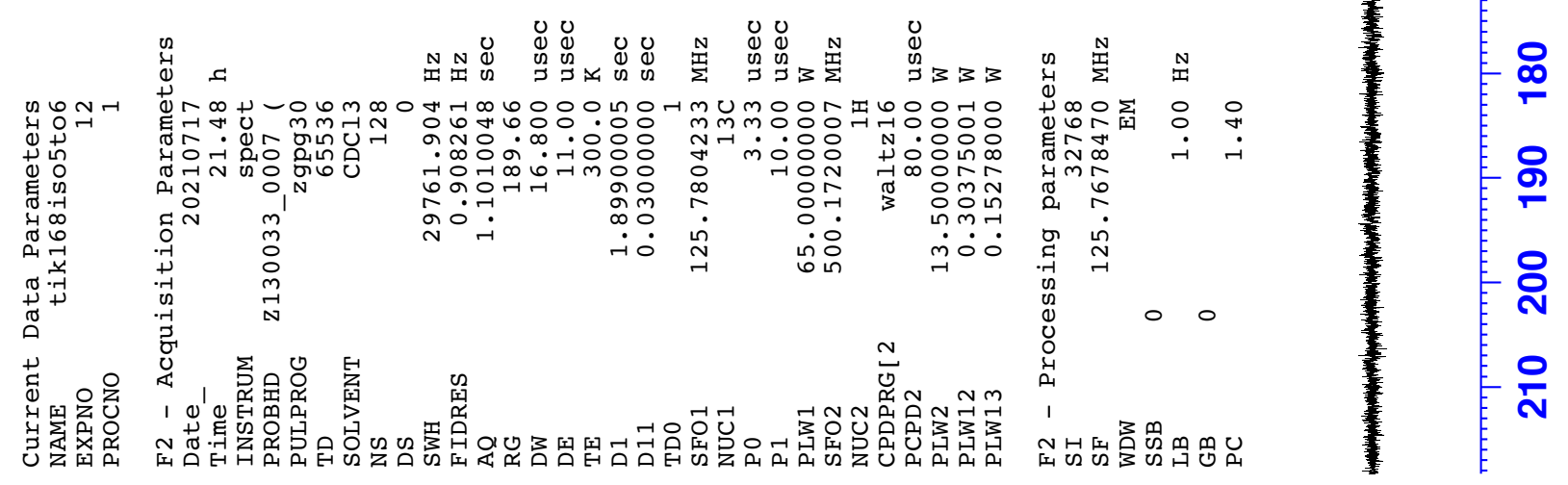


ธ96 0 $\left.0 \angle 6^{\circ} 0\right]$

$886^{\circ} 0$

โ โ $0^{\circ}$ [ ]

与 $80^{\circ}[\mathrm{T}$

$\mathrm{S} 60^{\circ}[]$

$8[\tau \cdot[]$

$6 \varepsilon[\cdot[]$

$\angle S[\cdot[]$

$\mathrm{SLI \cdot [}]$

$\angle 8 I \cdot \mathrm{I}$

$6 \mathrm{G} \varepsilon^{\circ} \mathrm{T}$

$98 \varepsilon^{\circ} \mathrm{T}$

$8 \mathrm{LG}^{\circ} \mathrm{T}$

$885^{\circ} \mathrm{L}$

$565^{\circ} \mathrm{T}$

ธ29. $\mathrm{I}$

โ $\varepsilon 9^{\circ}$ T

$\varepsilon \nabla 9^{\circ}[-$

$09^{\circ} \mathrm{L}$

I 0 I $\mathrm{Z}$

$80 \mathrm{~L} \cdot \mathrm{Z}-$

与 I $\tau \cdot Z-$

$\varepsilon Z \tau \cdot Z-\mu$

$L \varepsilon[\cdot \tau]$

$\varepsilon \nabla T \cdot Z-$

ISI $Z$.

$8 \mathrm{~S} \cdot Z-$

S9I・Z

ZLI Z

$08 \tau \cdot \tau$

$\nabla 29^{\circ} \varepsilon$

$708^{\circ} \mathrm{s}$

ฤ $28^{\circ} \mathrm{s}$

$Z L I \cdot L$

$8 L T \cdot L$

$6 L T \cdot L$

$\angle 8 I \cdot L-$

I6 $\mathrm{I}^{\circ} L$

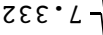

$9 \varepsilon \varepsilon \cdot L$

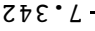

$\left.\varsigma \varpi \varepsilon^{\bullet} L\right\urcorner$

[ $\left.\subseteq \varepsilon^{\circ} L\right]$

ऽ૬ $\left.\varepsilon^{\bullet} L\right]$

โ $9 \varepsilon^{\circ} L$

५ $9 \varepsilon^{\circ} L-$

$\left.69 \varepsilon^{\circ} L\right]$

$S L \varepsilon^{\circ} L \backslash$

$\angle L \varepsilon^{\circ} L$

$8 L \varepsilon^{\cdot} L$

โ $8 \varepsilon^{\circ} L$

$\varepsilon 6 \varepsilon^{\circ}$

$\varsigma 6 \varepsilon \cdot L-$

$S 00^{\circ} L J$

$80 \sigma^{\circ} L J$

โS币

$9 S D^{\circ} L$

ธ9 $0^{\circ} L$

$89 \pi^{\circ} L-$

$\varepsilon \angle \nabla^{\circ} L-$

$08 \nabla^{\circ} L-$

$\varepsilon 87^{\circ} L$

$987^{\circ} L$

$L T L \cdot L$

$0 Z L \cdot L$

$\nabla Z L \cdot L-$

$\varpi \varepsilon L \cdot L$

$9 \varepsilon L \cdot L-$

$0 \nabla L \cdot L]$

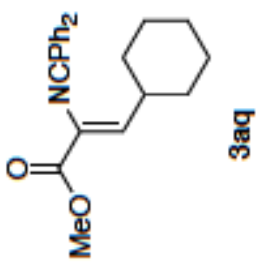

惌

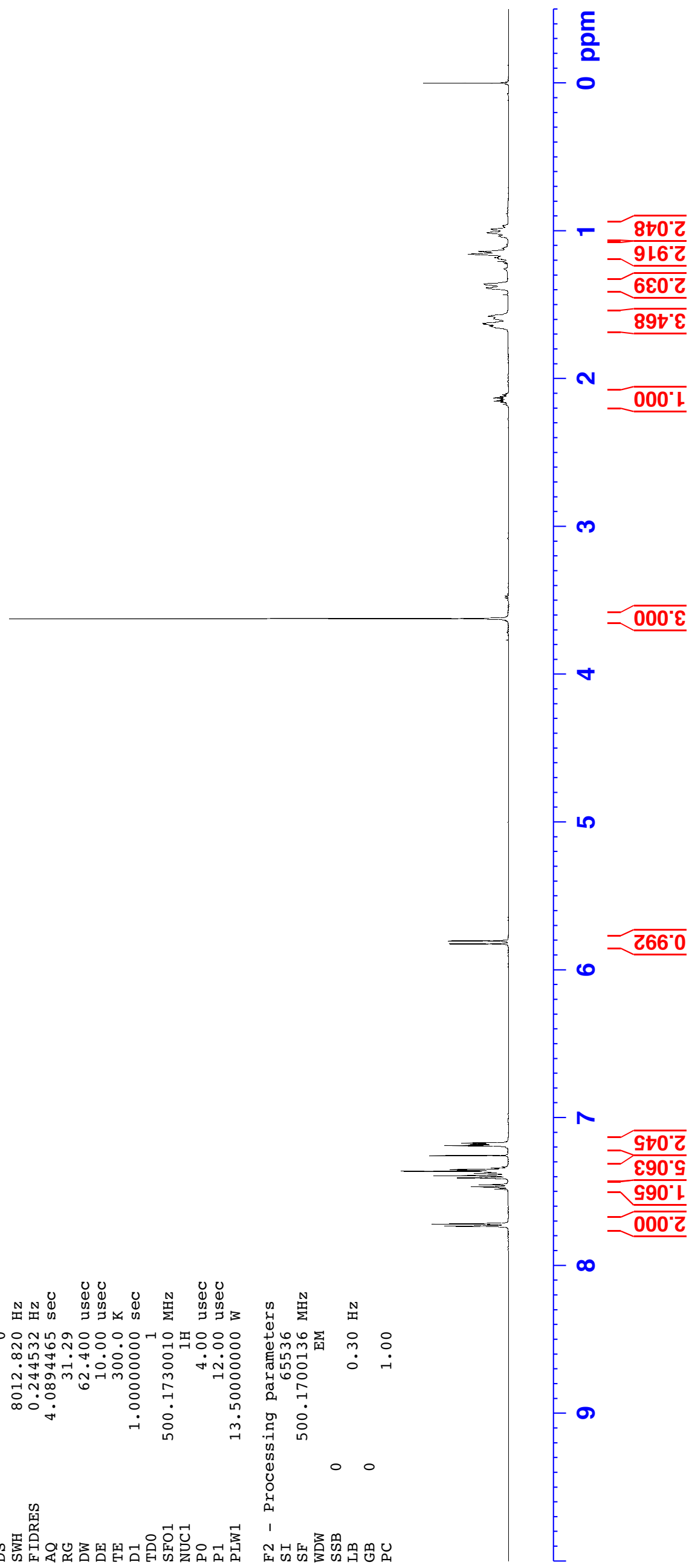

总

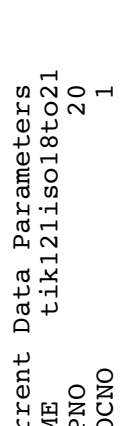

号

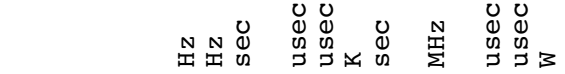

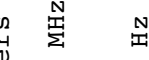

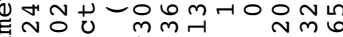

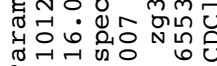

ल त

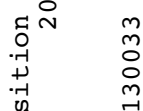

$m$
Oे
0
$m$
$\stackrel{N}{N}$

प्ष

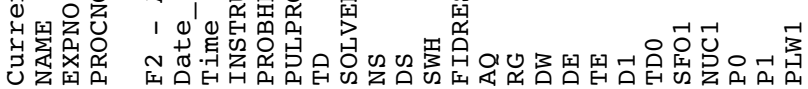

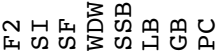



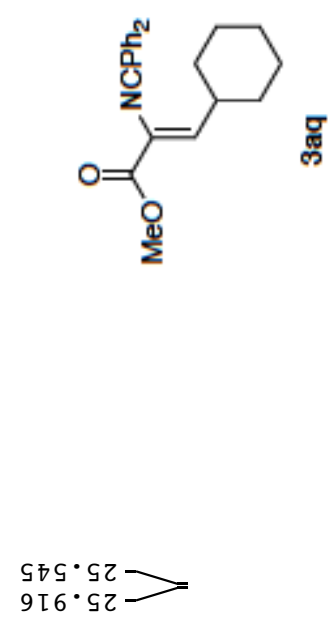

乙โゅ โ

$99 \varepsilon \cdot 9 \varepsilon-$

乙ฤ8・ โG

$\left.\varepsilon \angle 6^{\circ} \angle Z T\right]$

S8Z.8ZI

万LO०6ZI

Z8०. 6ZT

โ $89^{\circ} 0 \varepsilon \mathrm{L}$

$608^{\circ} 0 \varepsilon \mathrm{L}$

โ $9 L \cdot 9 \varepsilon \mathrm{L}$

$00^{\circ} 8 \varepsilon \mathrm{T}$

५ $80^{\circ} 6 \varepsilon \mathrm{I}$

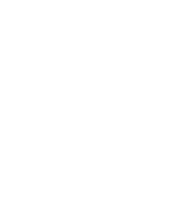

틍 

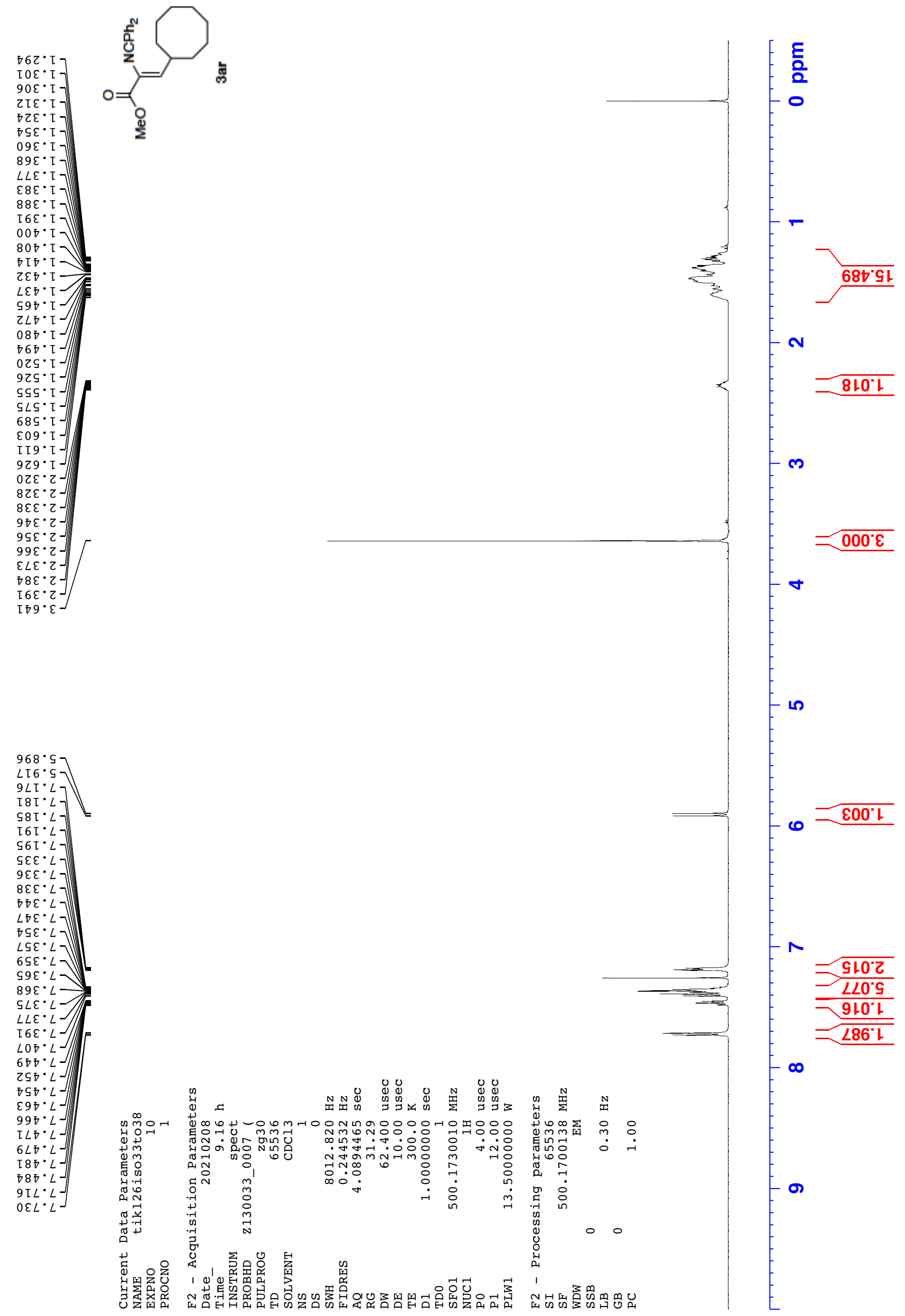

先 1

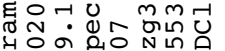

r.

I

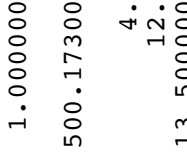

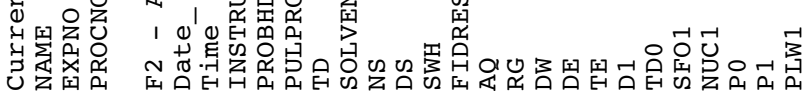

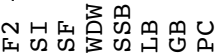



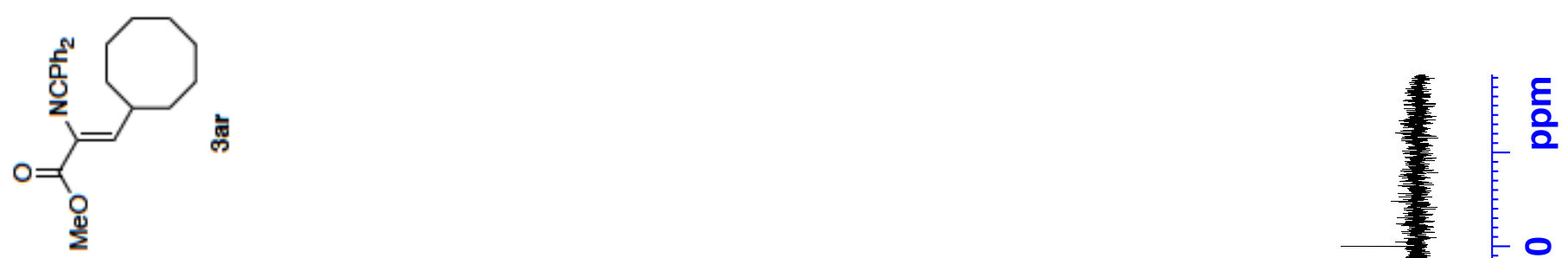

$\varepsilon \varepsilon[\cdot \textrm{ }$

$080^{\circ} 9 z$

โ $9 \mathrm{I} \cdot \mathrm{LZ}$

$0 \angle 9^{\circ} 0 \varepsilon-$

$6 \succsim 8 \cdot 9 \varepsilon$

$098^{\circ}$.

$866^{\circ} \angle Z T$

$0 \mathrm{SI} \cdot 8 \mathrm{Z}$

$992 \cdot 8 Z \mathrm{I}$

$90 \mathrm{I} \cdot 62 \mathrm{I}$

0马ซ $62 \mathrm{~T}$

$96 L^{\circ} 0 \varepsilon \mathrm{I}$

† $\angle 9^{\circ}$ โ $\varepsilon \mathrm{L}$

$62 L \cdot 9 \varepsilon \mathrm{T}$

$066^{\circ} 9 \varepsilon \mathrm{L}$

Zโ โ $6 \varepsilon \mathrm{L}$

$\varepsilon 6 \sigma^{\circ} \varsigma 9 \mathrm{~L}$

$0 \tau \varepsilon \cdot 0<\tau$

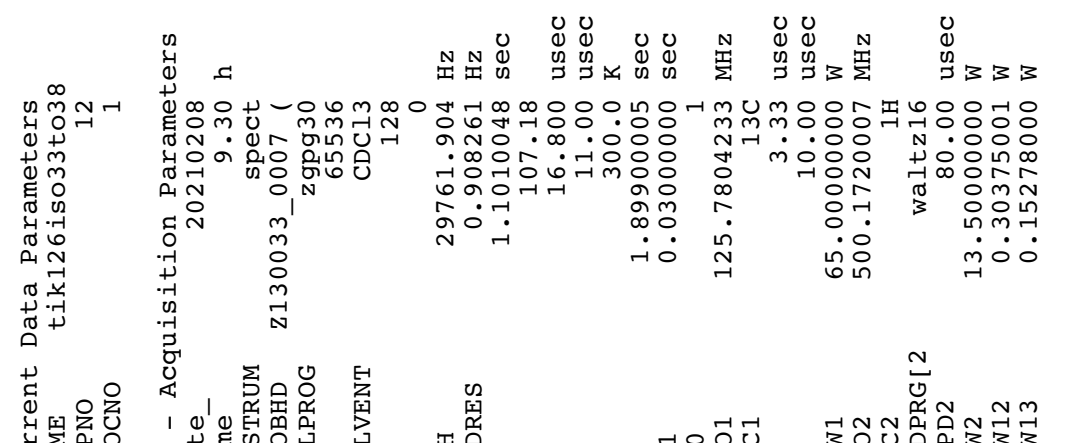

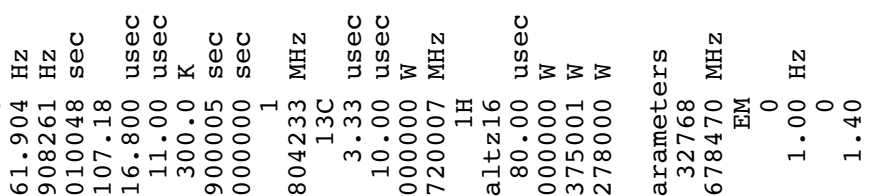

ำ

กิ

요

우

오

8

옹

$\infty$

8

8

으

ลิ

옴

운

옴

8

온

:

옹

С્

은

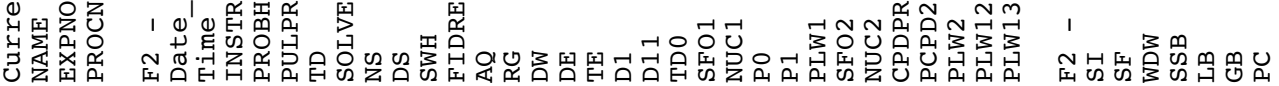



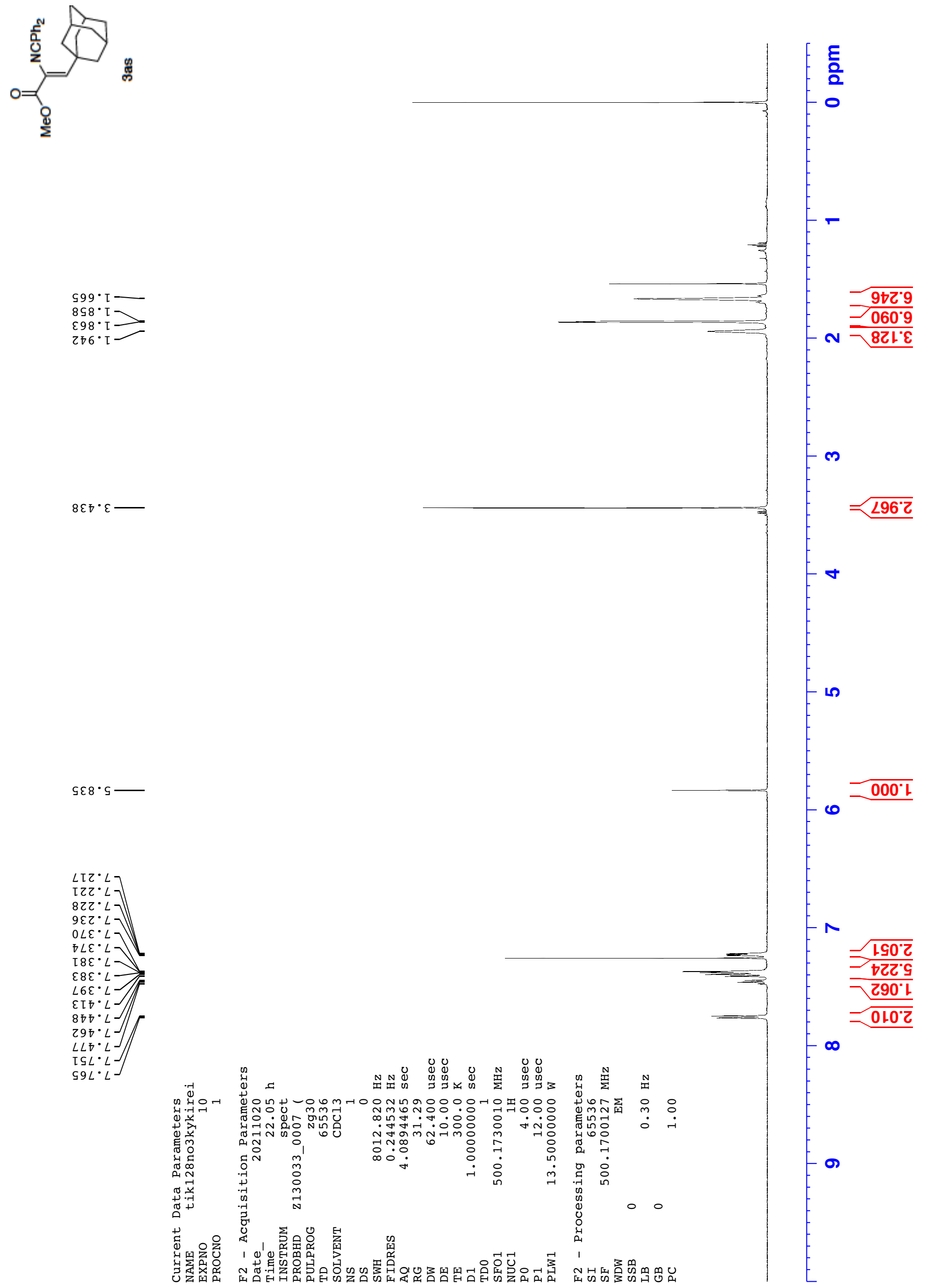

墭

$\varsigma \varepsilon 8 \cdot \varsigma$

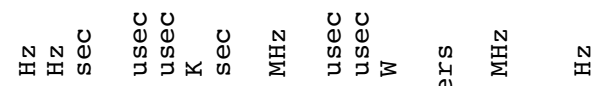
$898^{\circ} \mathrm{T}$

$\varepsilon 98^{\circ} \mathrm{T}$

乙จ $6^{\circ} \cdot$

$8 \varepsilon \mp \cdot \varepsilon-$

$\angle T z^{\circ} \angle$

$822 \cdot L]$

$9 \varepsilon 2 \tau^{\circ} L$

$\left.\nabla L \varepsilon^{\circ} L\right]$

I $8 \varepsilon^{\circ}\llcorner$

$\angle 6 \varepsilon \cdot L-$

$\varepsilon[\hbar \cdot L J$

$8 \nabla \sigma^{\circ} L J / /$

2

[SL $L J]$ 

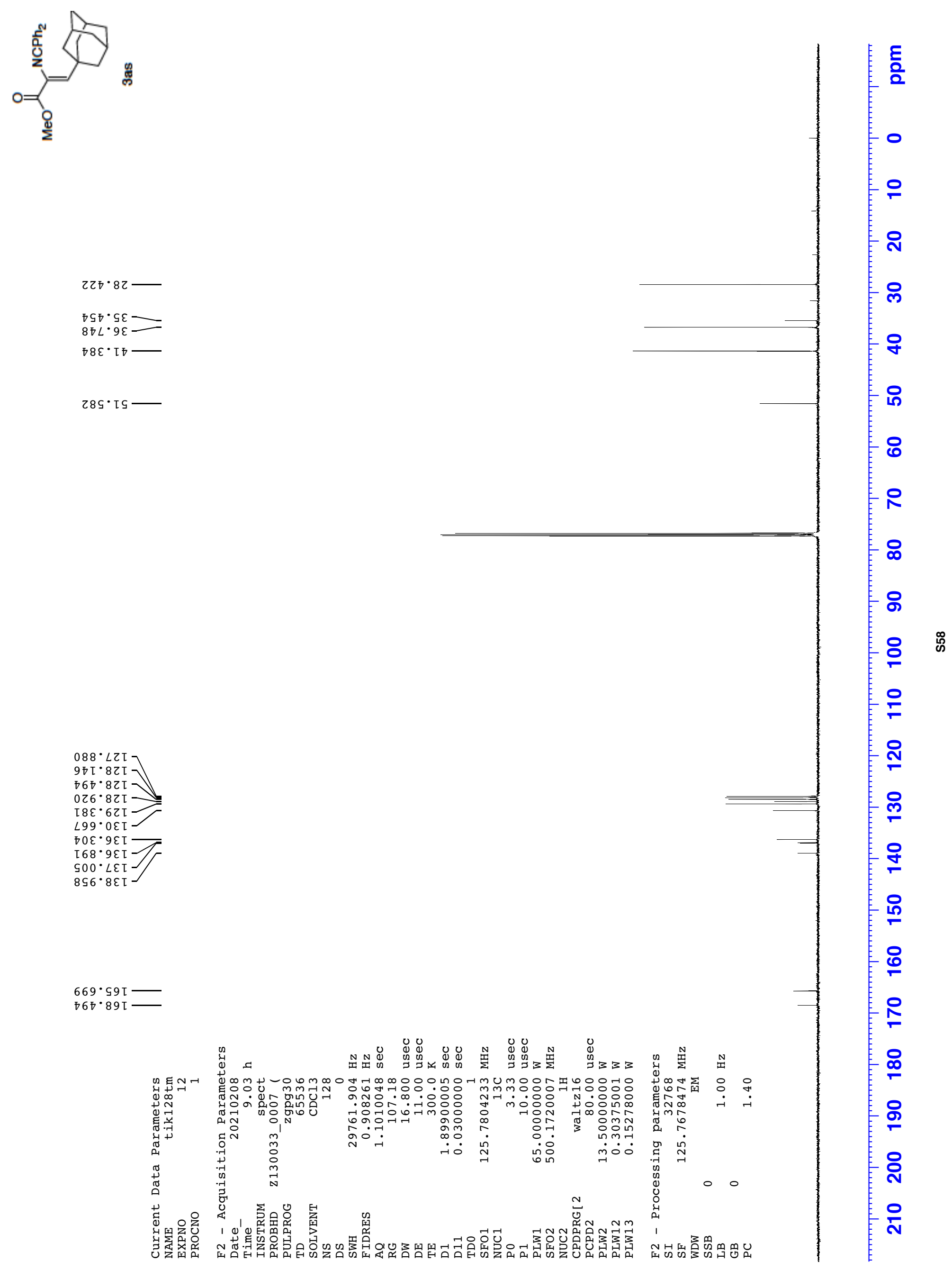

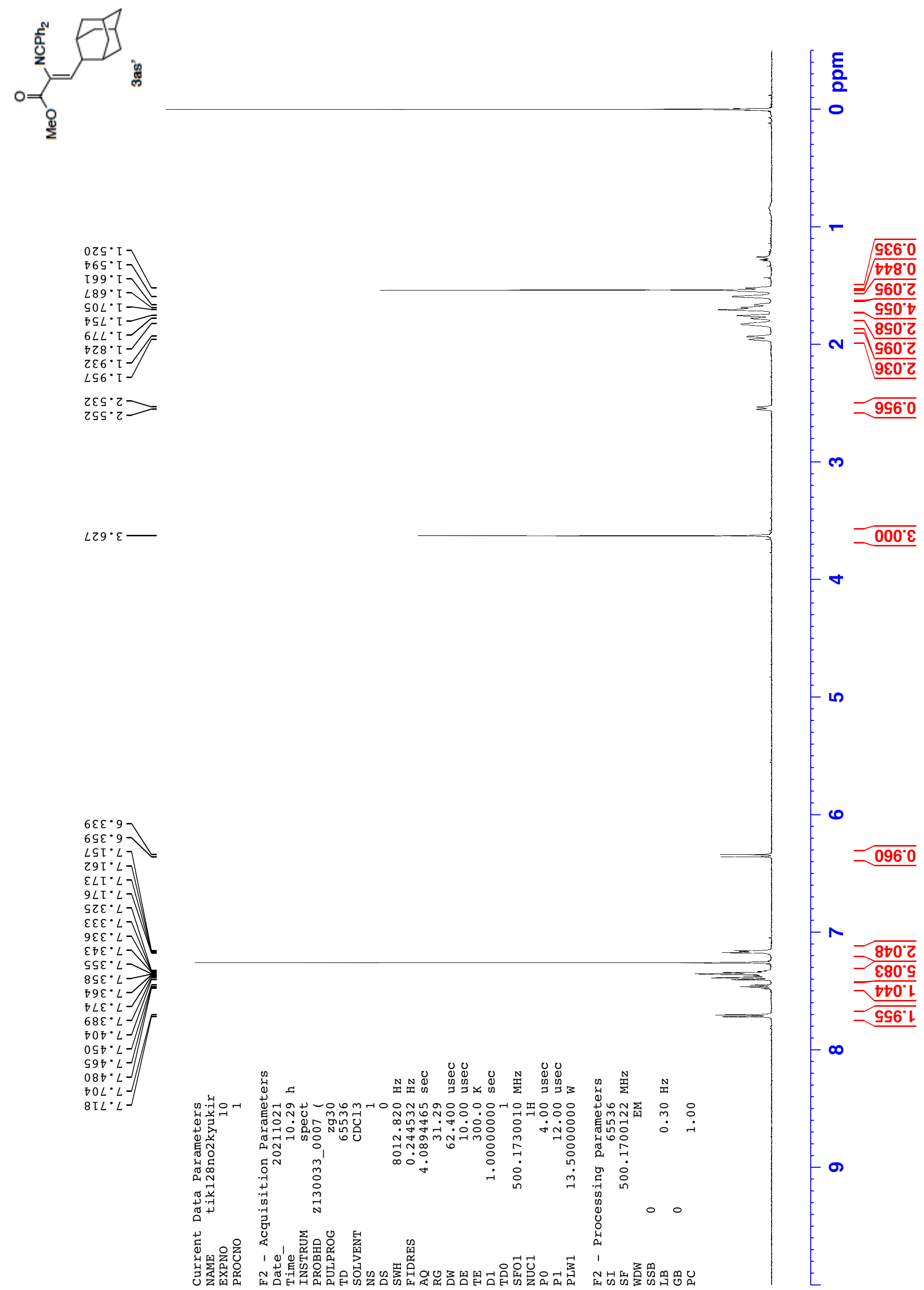

品

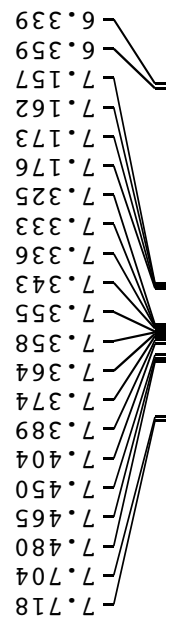

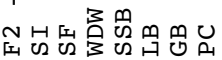



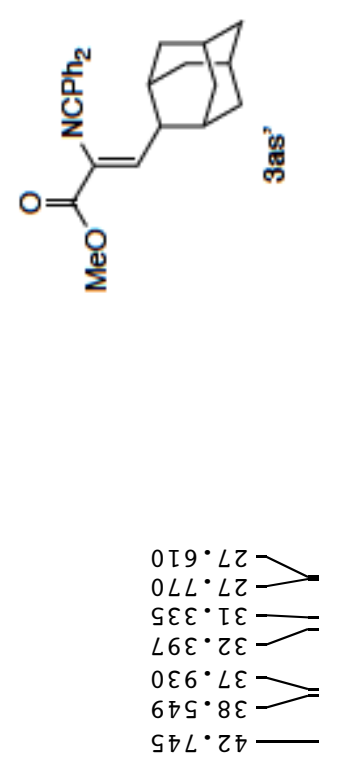

$8 \varepsilon 8^{*} \mathrm{TS}$

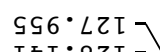

โ万โ $82 \tau$

S9L.8ZI

$\varepsilon \angle 0^{\circ} 6 Z \mathrm{~T}$

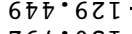

Z6L ${ }^{\circ} 0 \varepsilon \mathrm{L}$

$8 \varsigma 8^{\circ} 9 \varepsilon \mathrm{L}$

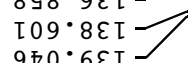

乙๐て $99 L$

$\varsigma \varepsilon I \cdot 0 L I-$

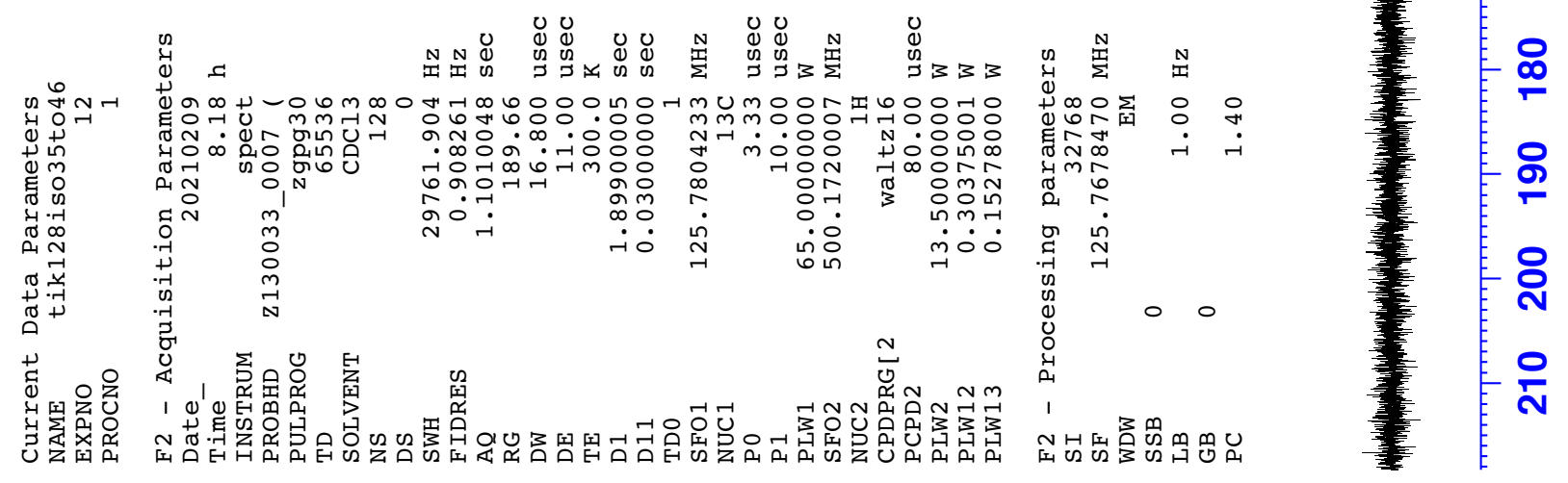



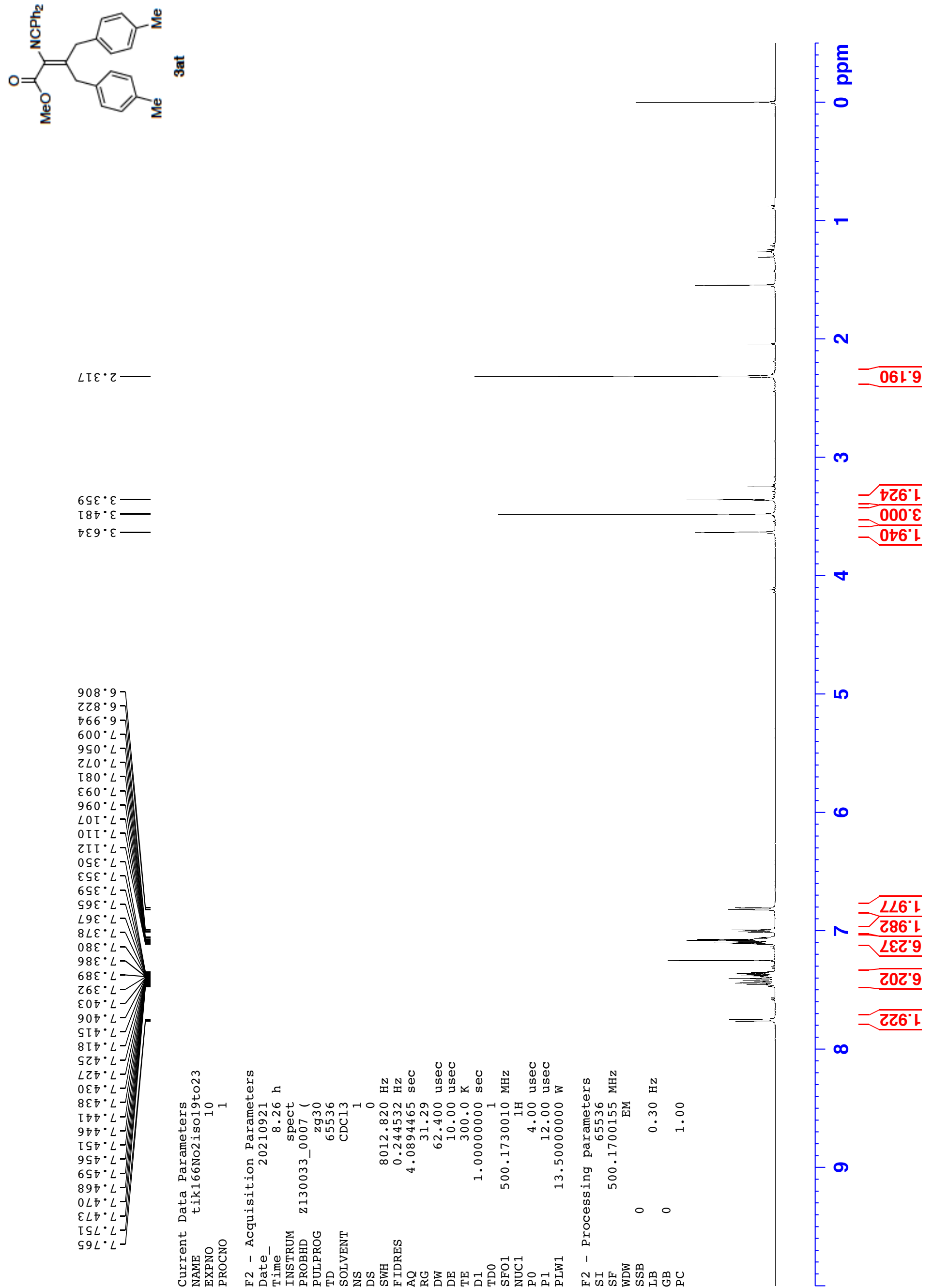


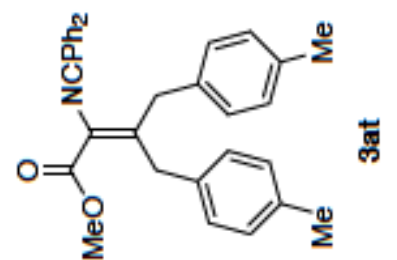

흠

Ђ૬ $0^{\circ}$ โ乙

$\tau L L \cdot G \varepsilon>$
$6 L \varepsilon \cdot 9 \varepsilon$

โ૬ $\mathcal{E}^{\top} \tau \varsigma$

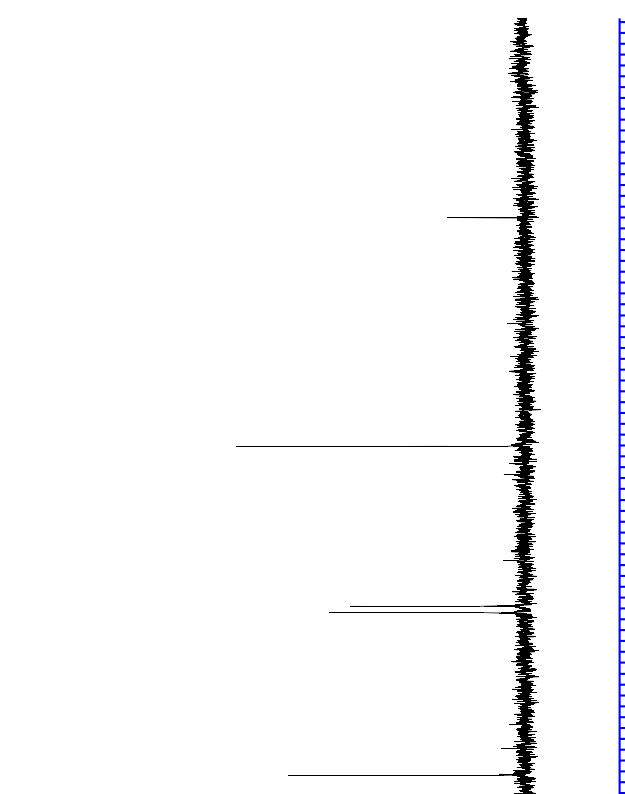

$\varepsilon 0 \varepsilon \cdot \varsigma \varepsilon \tau-$

$\varepsilon \tau 9^{\circ} \varsigma \varepsilon \tau-$

โ L $\tau \cdot 9 \varepsilon \tau$

ZES $9 \varepsilon \tau-$

$\angle 00^{\circ} L \varepsilon T-$

Z $\angle 0^{\circ} \angle \varepsilon T$

$06 \mathrm{C}^{\circ} \angle \varepsilon T$

$8 \subseteq 8 \cdot 8 \varepsilon[$

0 IS. 99 L

$0 \subseteq 6 \cdot 69 \mathrm{I}$ 


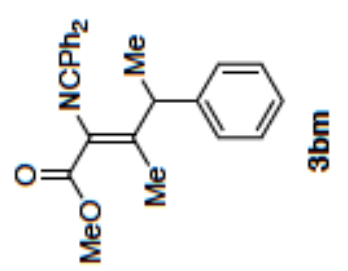

$\varepsilon \varepsilon \varepsilon \cdot \tau$

$L L \varepsilon^{\circ} T-$

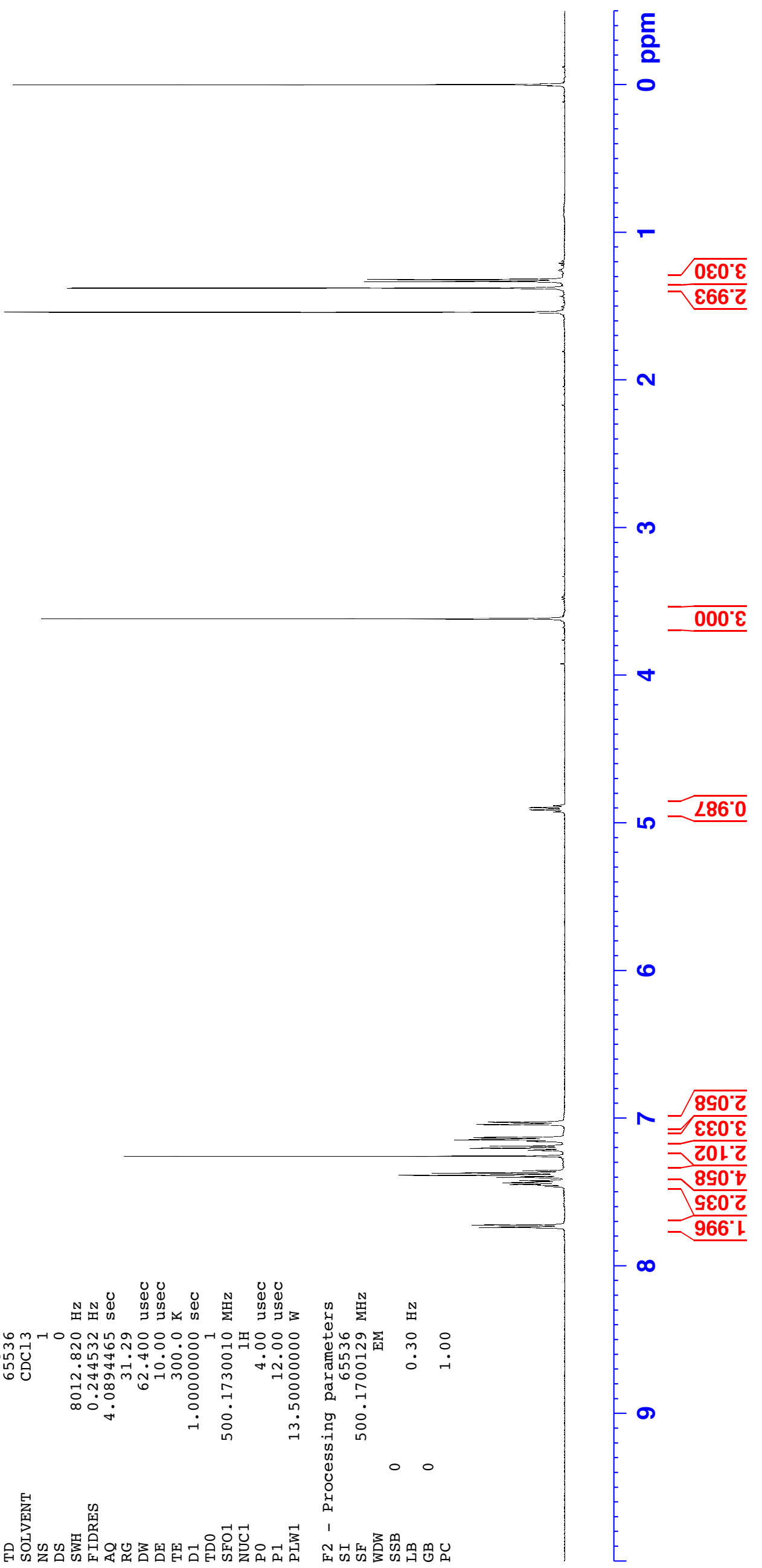

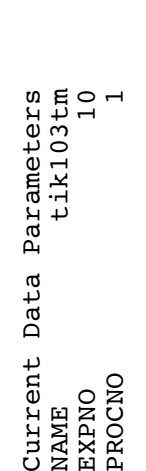

$\stackrel{0}{0}$

$8[9 \cdot \varepsilon$

$\varepsilon 88^{\circ} \mathrm{T}$

$\angle 68^{\circ} \mathrm{T}$

โ $16^{\circ}$.

$\angle Z 0^{\circ} L$

$0 \varepsilon \tau \cdot L$

$\varepsilon \varepsilon \tau \cdot L$

$\nabla \nabla[\cdot L]$

$\angle D[\cdot L]$

$8 \mathrm{SI} \cdot L]$

万OZ $L$

$8 \mathrm{I} Z^{\circ} L$

$\varepsilon L \varepsilon \cdot L$

$\angle 8 \varepsilon^{\circ} \angle$

$20 \sigma^{\circ} \circ$

$\angle Z \nabla \cdot L]$

$\operatorname{SE} 0^{\circ} L J$

OSE $\cdot L]$

$\triangle 5 D^{\circ} L$

万9D $\angle L$

$O \nabla L \cdot L]$
퉁ำ ำ

至

⿷匚⿳丨コ丨

$\begin{array}{ll}-0 & m \\ -1 & 0 \\ -7 & 0 \\ -7 & 0 \\ -1 & -1\end{array}$

उु

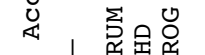

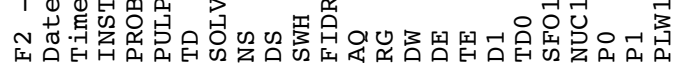

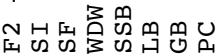

ஜூ

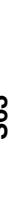

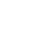




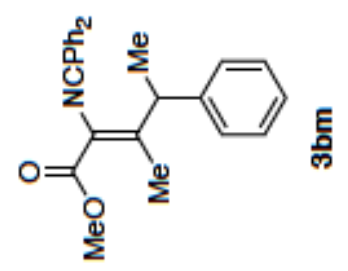

Z8E・ T -

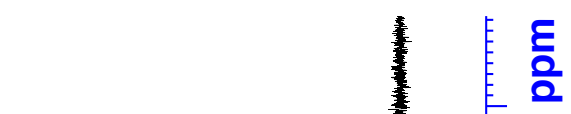

$20 S \cdot 8 \varepsilon$

ฤธ๊ โS

$69 L \cdot G Z$

$988^{\circ} \angle Z T$

$8 \mathrm{~S}^{\circ} \mathrm{LZI}$

$260^{\circ} 8 Z \mathrm{I}$

9万I. $82 \mathrm{I}$

$700 \cdot 6 Z \mathrm{~T}$

万6I $62 \mathrm{I}$

乙08 $0 \varepsilon \mathrm{L}$

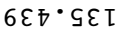

$\varsigma 6 \mathrm{C}^{\circ} \mathrm{LE} \mathrm{I}$

$8 L \mathrm{~L} \cdot 8 \varepsilon \mathrm{L}$

$6 \mathrm{~T} 6^{\circ} 8 \varepsilon \mathrm{L}$

$9 \varepsilon \varsigma^{\circ} \varepsilon \nabla \mathrm{L}$

$\angle 0 Z \cdot S 9 \tau$

$0 \varpi 乙 \cdot 0 L I$

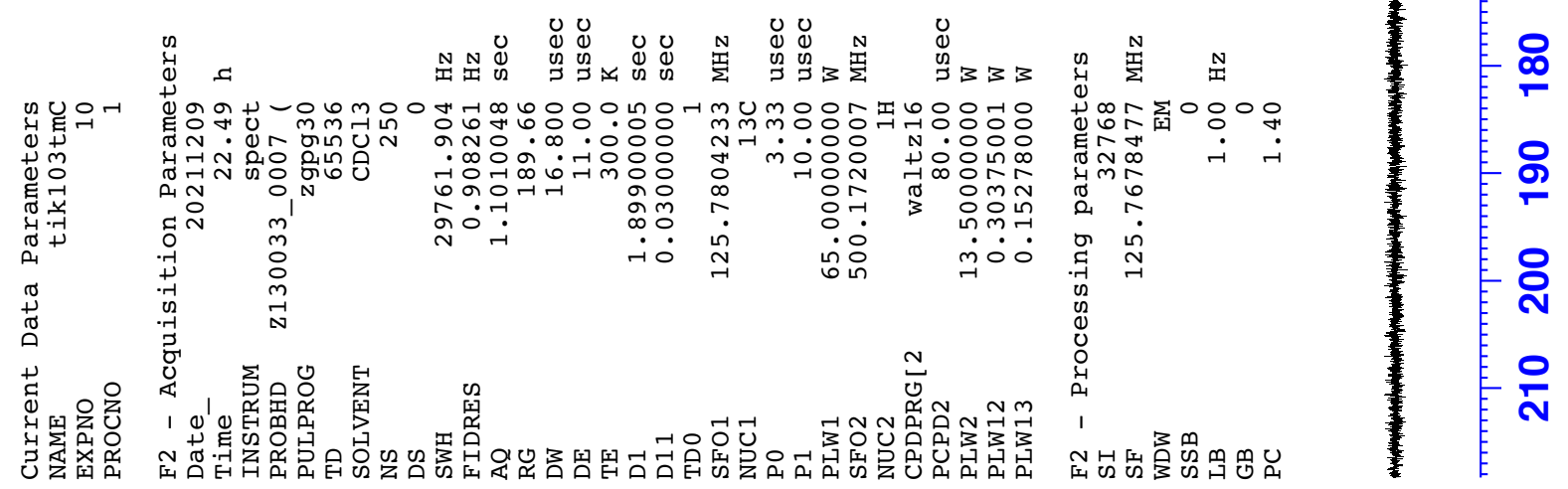



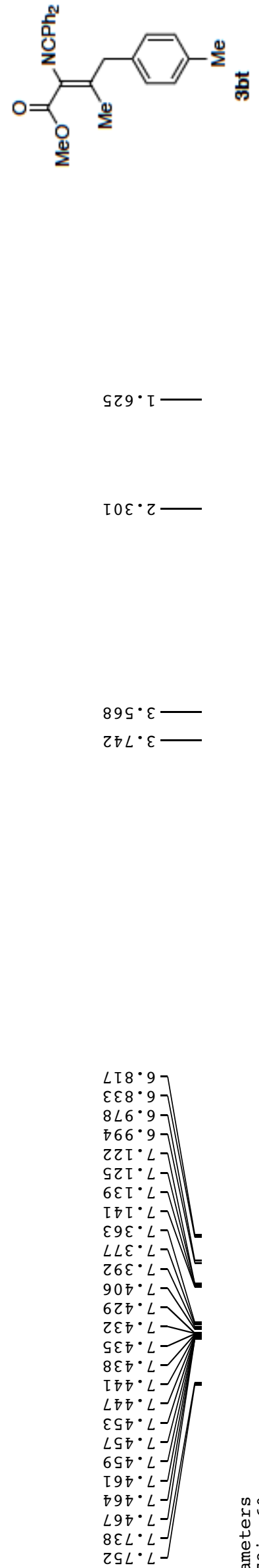

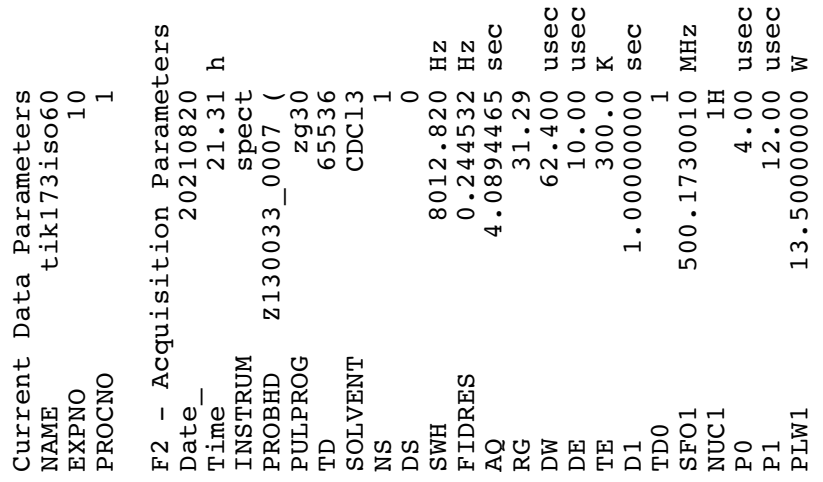

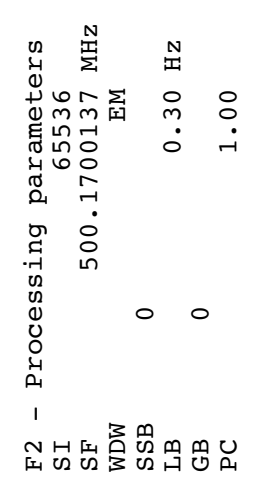

@્ 


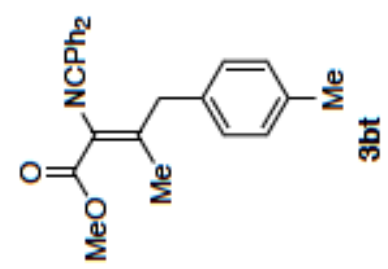

$80 \varepsilon \cdot 6 \tau=$
$920 \cdot \tau 2=$
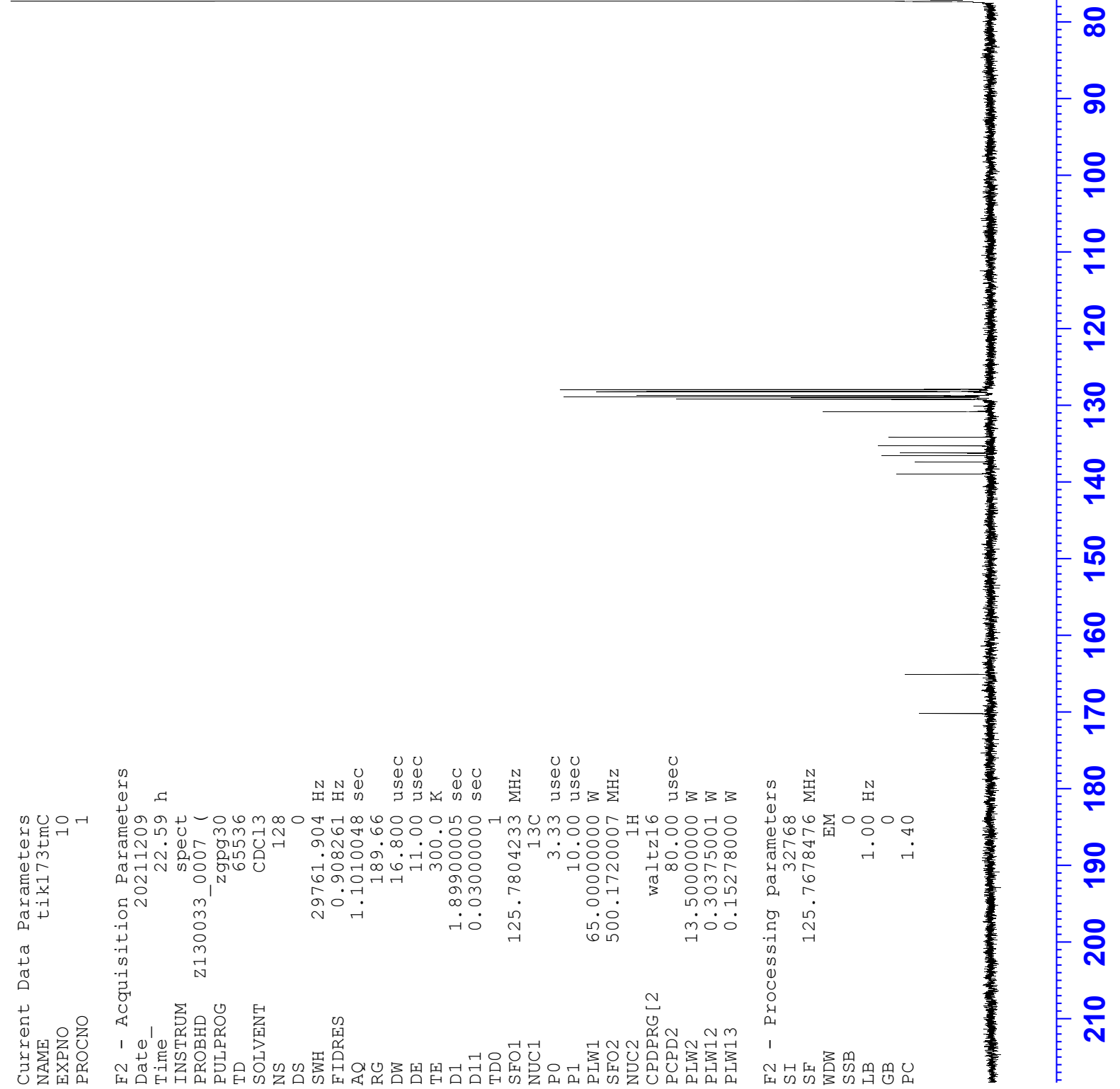


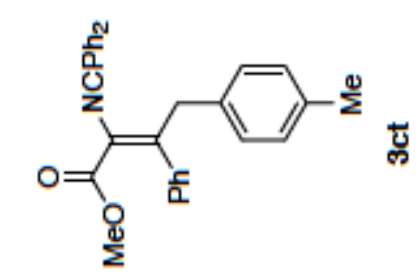

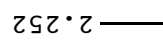

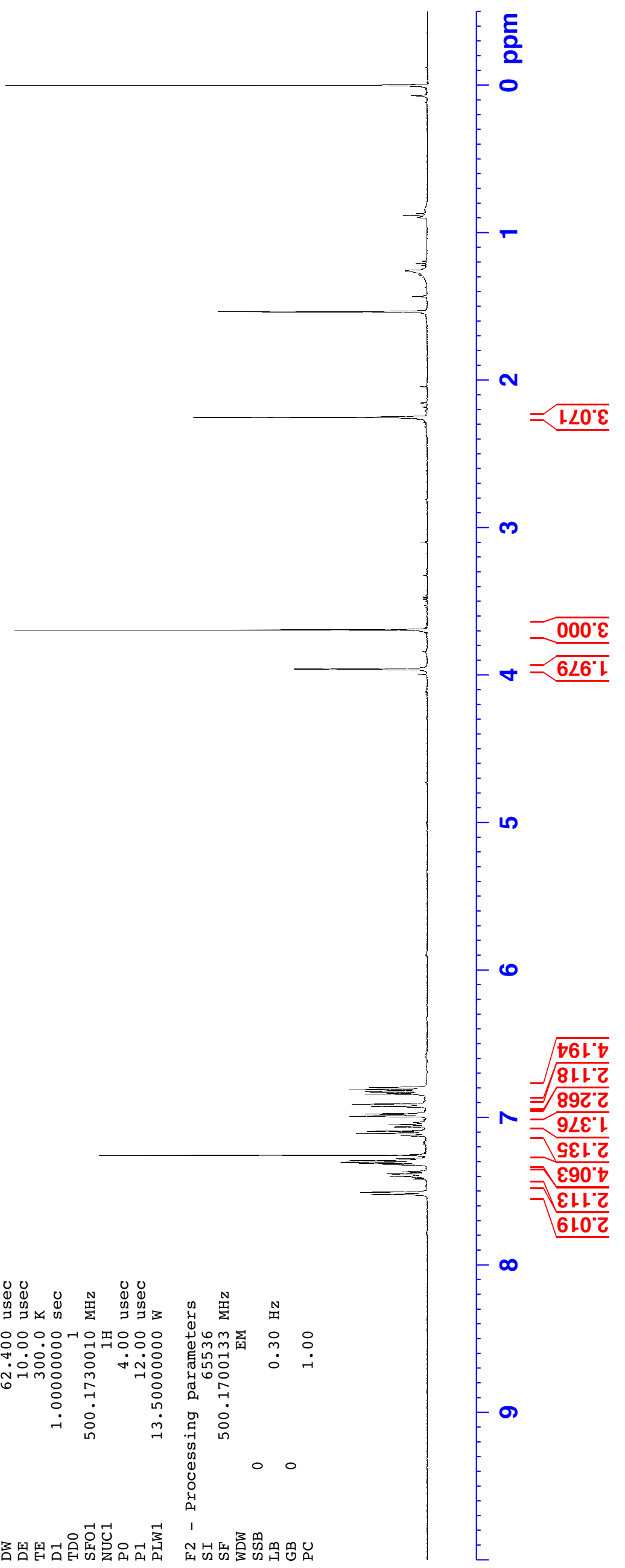

$\hat{\oplus}$

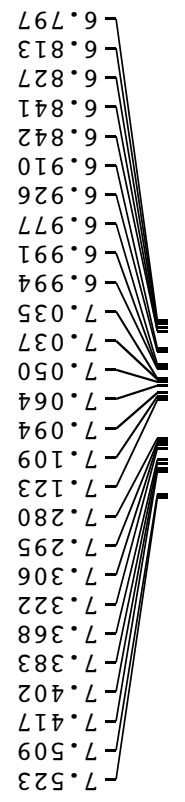

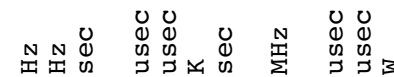
$\nabla 69^{\circ} \varepsilon$ $6 \varsigma 6 \cdot \varepsilon-$ ENล근

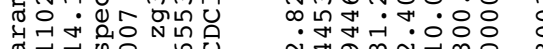

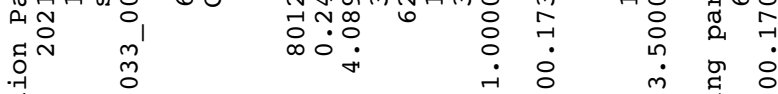
-7 $\quad$ त

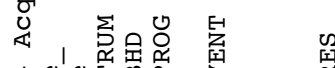

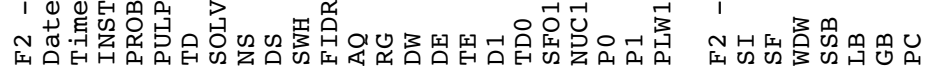




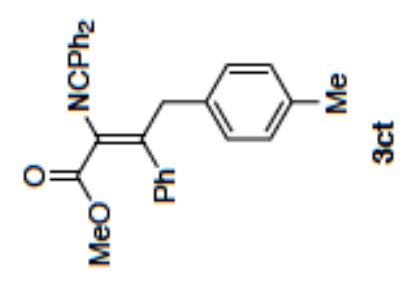

흘

$\tau 00^{\circ} \tau Z$

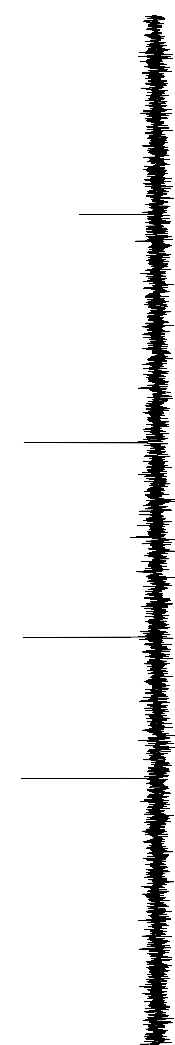

$8 \tau 6 \cdot 8 \varepsilon$

9 I $6^{*}$ โ

$\angle 9 L \cdot 9 Z[$

$\angle O S^{\circ} \angle Z T$

$8 \subseteq 8^{\circ} \angle Z I$
$S 66^{\circ} \angle Z I$

乙乙८・8乙

$70 \mathrm{~S} \cdot 8 Z \mathrm{~L}$

$8 \nabla L \cdot 8 Z T$

$206^{\circ} 8 Z \mathrm{I}$

$996^{\circ} 8 Z \mathrm{I}$

$58 \mathrm{C}^{\circ} 6 \mathrm{ZT}$

S09.0हा

SZ・ZE I

S07 $9 \varepsilon \tau-$

$\varepsilon 0 \tau \cdot 8 \varepsilon \tau-$

$8[6 \cdot 8 \varepsilon \tau$

乙E乙・ Oெ

I $\angle 6^{\circ}$ ऽ 9 I

$\angle 99^{\circ} 69 \mathrm{I}$

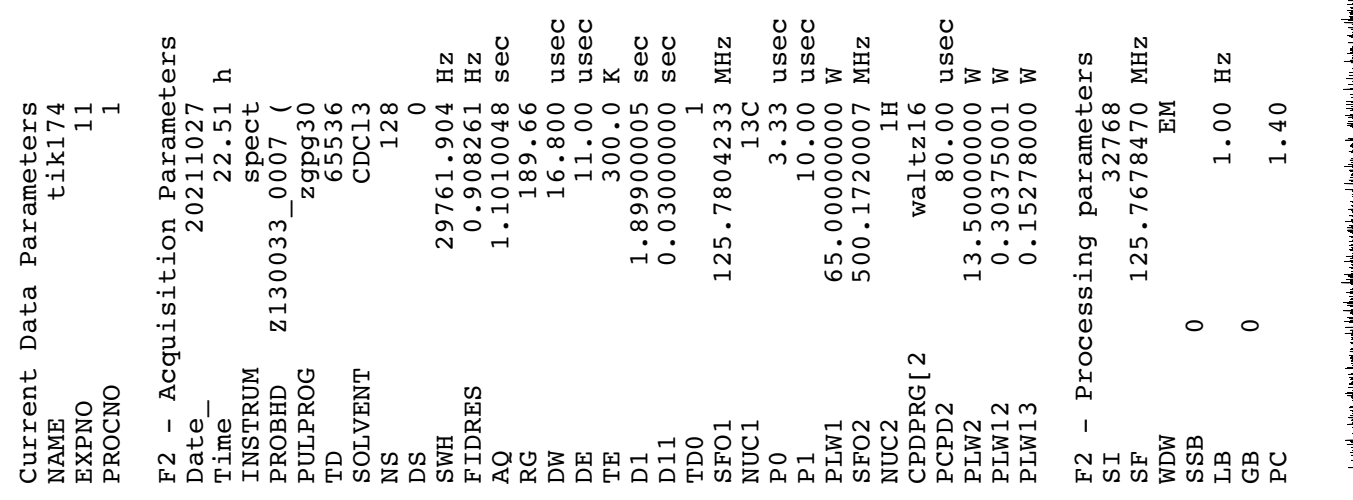




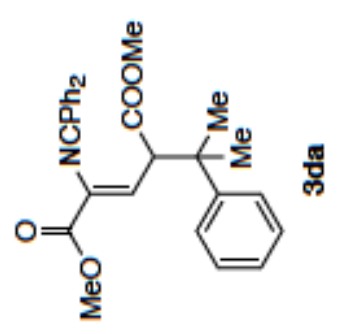

$\varepsilon \Phi^{\circ} \cdot T$
$\angle L D^{\circ}$

$\nabla \varsigma \mathcal{~} \cdot \mathcal{E}$

$\angle 6 \sigma^{\circ} \varepsilon$

$L L L \cdot \varepsilon \longrightarrow$

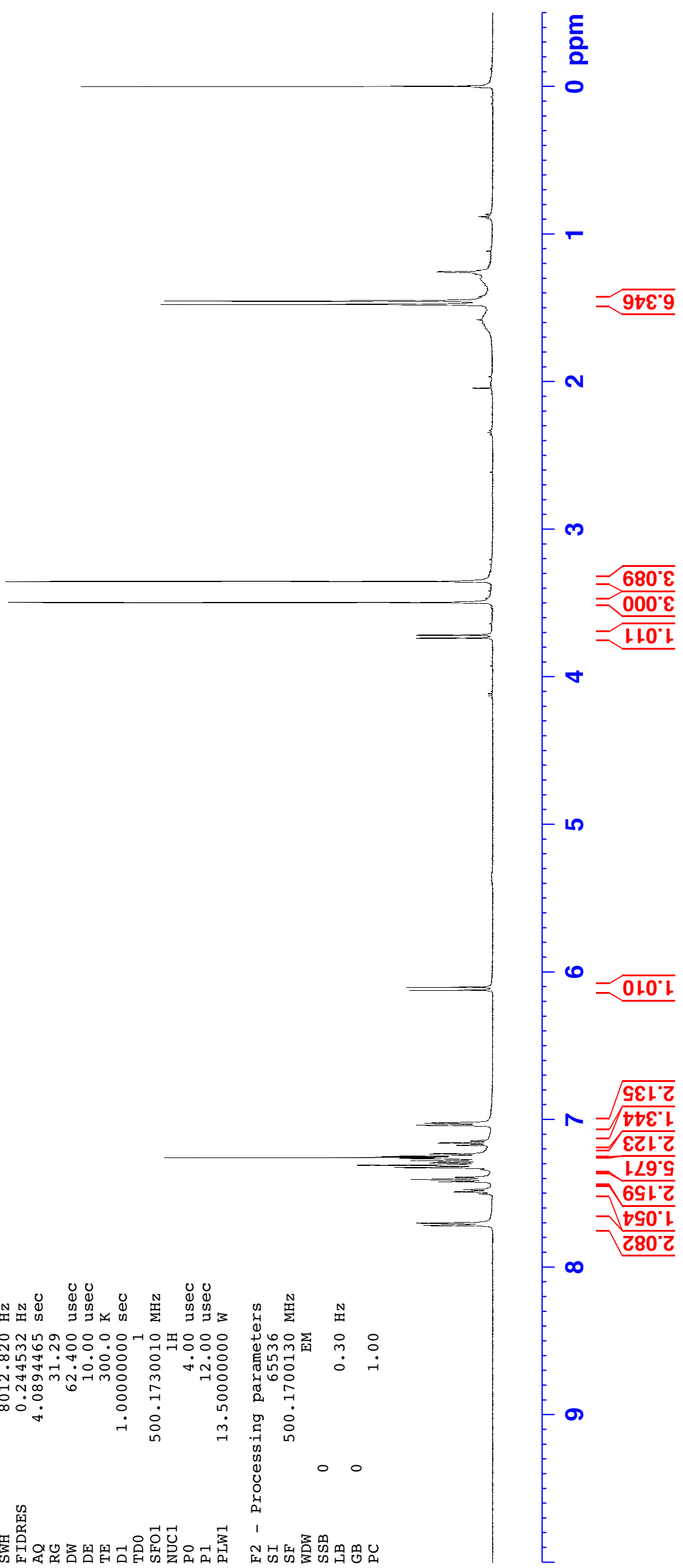

$\stackrel{8}{\circ}$

ธ0I $\cdot 9$

SZT:9

SZ0 $\left.0^{\circ} L\right]$

$9 \nabla[\cdot L]$

$09[\cdot L]$

$\left.\begin{array}{c}G L \\ \varepsilon \varepsilon \tau^{\circ} L \\ \hline\end{array}\right]$

$6 \pi \tau \cdot L]$

$79 z^{\circ} L \backslash V$

与 $6 \mathrm{C}^{\circ} L$

$60 \varepsilon^{\circ} L$

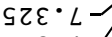

โ $\nabla \varepsilon \cdot L \perp$

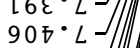

ZZ๐ $L J$

$9 \angle \nabla^{\circ} \angle J$

$06 \sigma^{\circ} L-$

$\varepsilon 0 L \cdot L-$

8I $L \cdot L]$

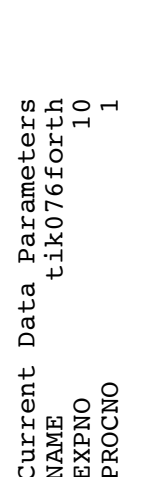

先

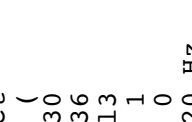
눅어

ज.

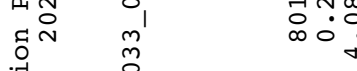

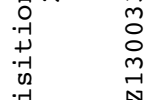

प

总星

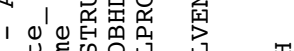

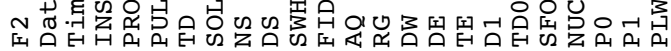

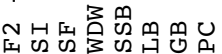




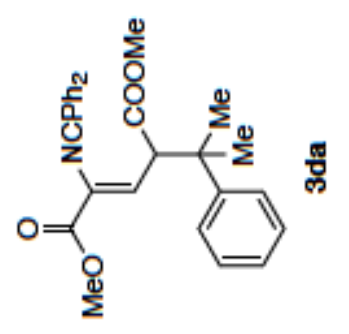

$8 \varepsilon 6 \cdot 97 \square$
$\varepsilon 8 \varepsilon \cdot 97$

$978 \cdot \operatorname{L\square 一}$

$Z 2 \cdot$ โ

$\angle Z 8^{\circ}$ โS

โธ9.๑马
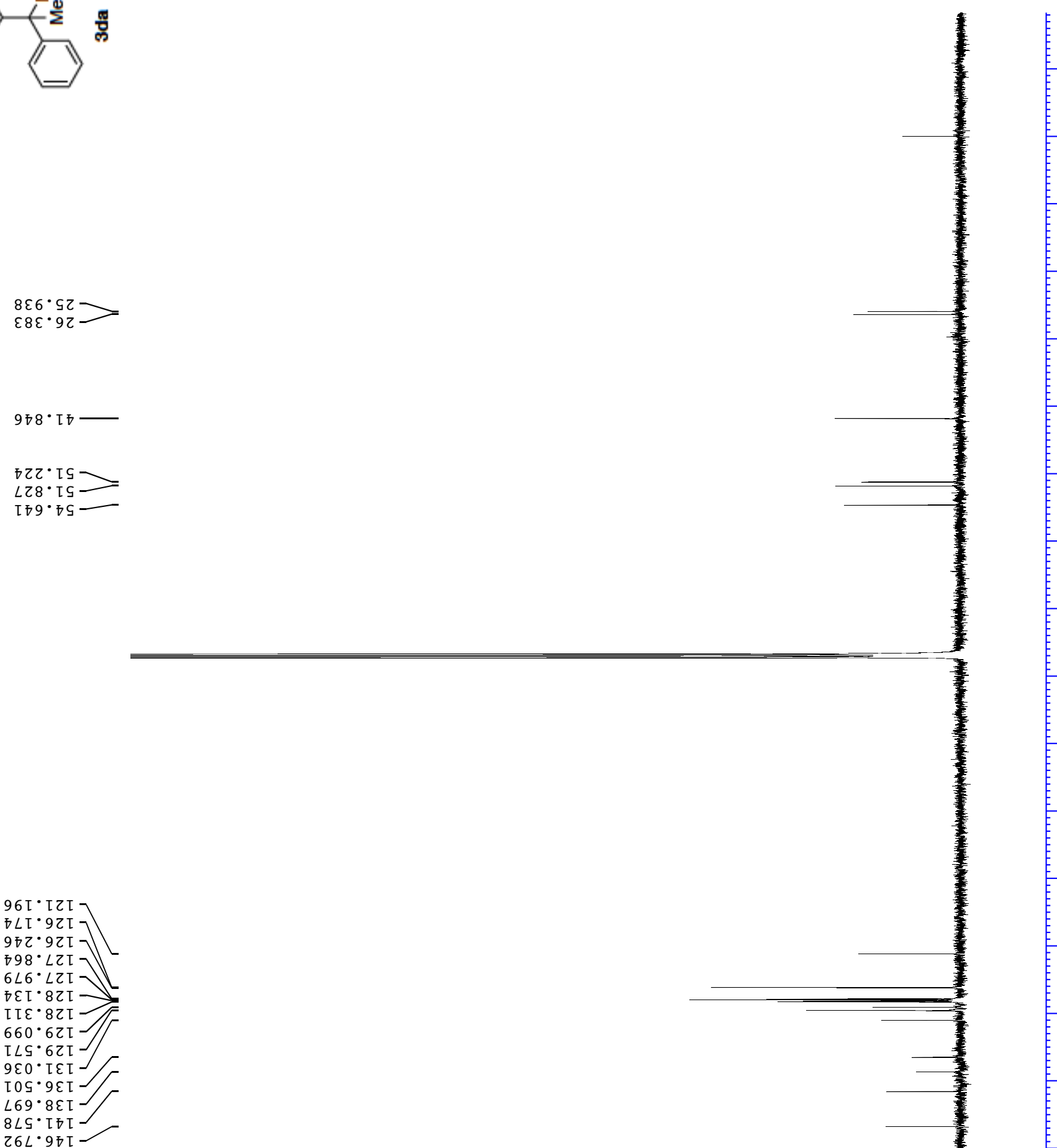

흠 


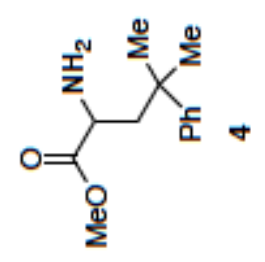

\begin{tabular}{l} 
S与E \\
0 โ \\
\hline
\end{tabular}

$\varepsilon \tau 8^{\circ} \mathrm{T}$

$\angle Z 8^{\circ} \mathrm{T}$

โธ $8^{\circ} \mathrm{T}$

५५ $8^{\circ} \mathrm{L}$

$\varepsilon\left[\tau^{\circ} Z\right.$

ऽてて 乙

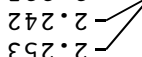

$\angle 0 \varepsilon \cdot \varepsilon>$

$8 \tau \varepsilon \cdot \varepsilon$

$0 乙 \varepsilon \cdot \varepsilon$

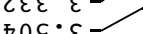

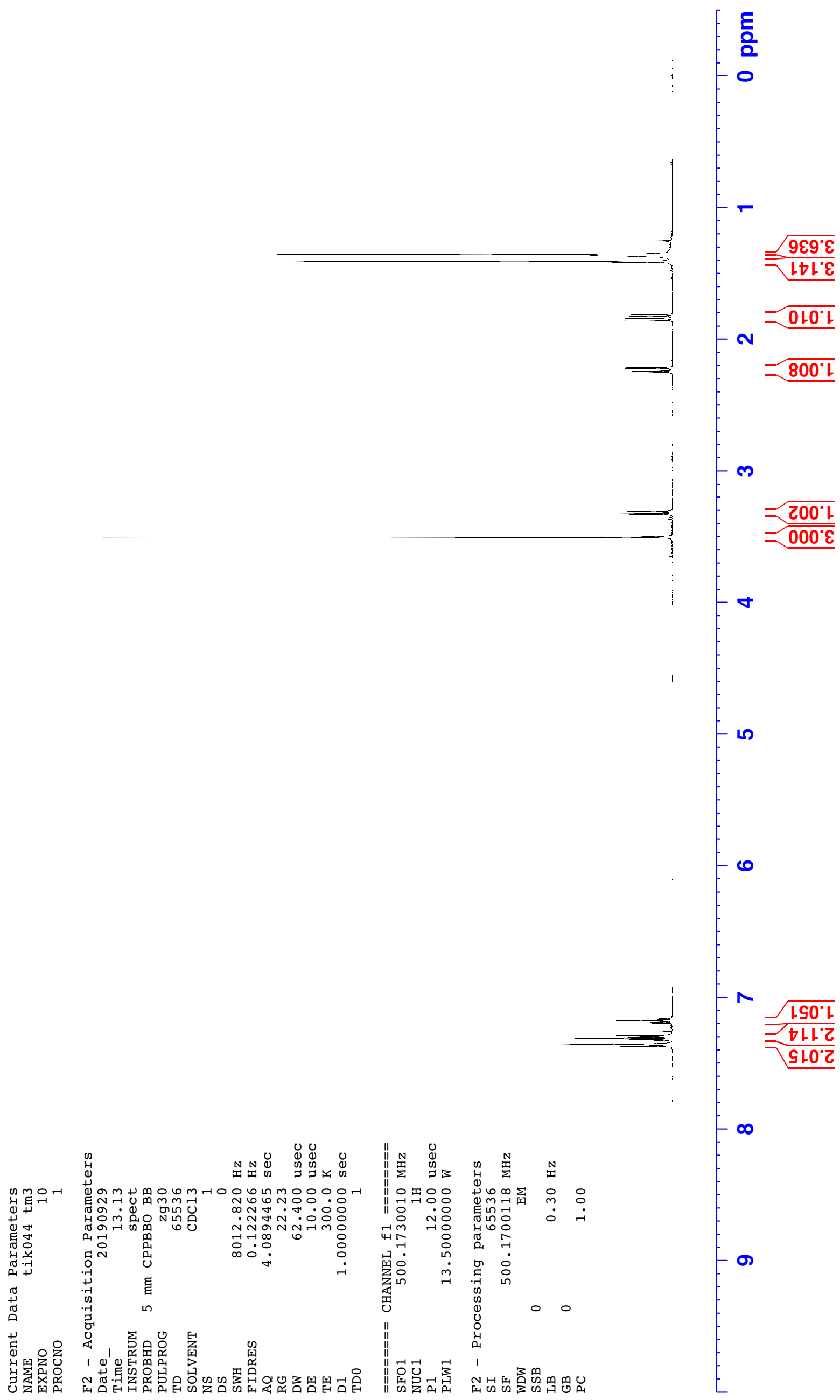

है

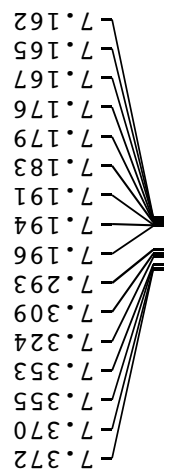




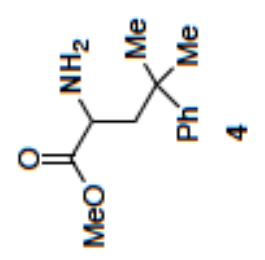

$982 \cdot 62>$

$0 \varepsilon \mathrm{s} \cdot 6 z$

$\varsigma I \varepsilon \cdot L \varepsilon$

$9 L \varepsilon \cdot 6 \bar{D}$

I08. IS

$928.9 L$

$080^{\circ} \angle L$

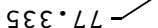

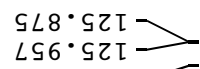

次

I $0 \cdot 8 \pi \mathrm{I}$

$6 L G \cdot 9 L I$

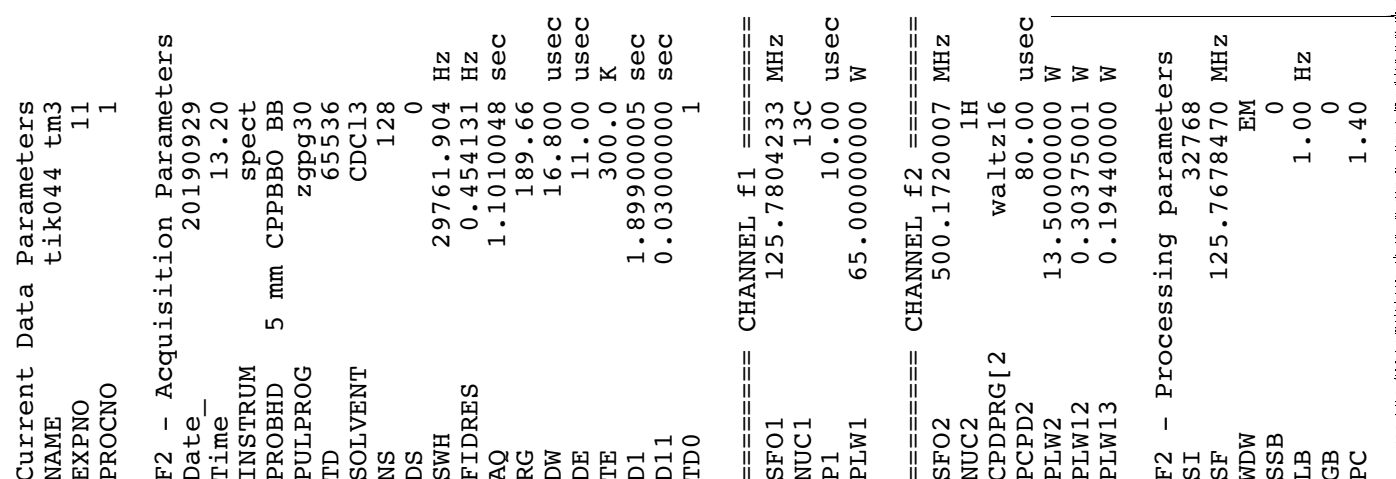

틍

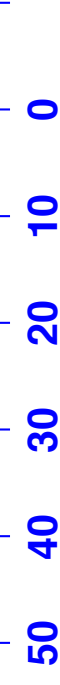

8

웅

$\infty$

ஃ

은 กิ

음

온

음

운

음

우

P

욷

요 